\title{
A [4 + 1] Cyclative Capture Access to Indolizines via Cobalt(III)-Catalyzed $\mathrm{Csp}^{2}$-H Bond Functionalization
}

Xun Chen, Xinwei Hu, Yuanfu Deng, Huanfeng Jiang, and Wei Zeng *

Key Laboratory of Functional Molecular Engineering of Guangdong Province, School of Chemistry and Chemical Engineering, South China University of Technology, Guangzhou 510641, China

Corresponding author:zengwei@scut.edu.cn

\section{Supporting Information}

\section{Table of Contents}

1. General Experimental Information.

1.1. Table 1. Catalyst screening for a $[4+1]$ cyclative capture access to indolizines................. 3

1.2. Table 2. The effect of $\mathrm{Ag}$ salts on a $[4+1]$ cyclative capture access to indolizines .........3

1.3. Table 3. The effect of additive on a [4 +1$]$ cyclative capture access to indolizines ...........3

1.4. Table 4 . The effect of solvents on a $[4+1]$ cyclative capture access to indolizines ...........4

1.5. Table 5 . The effect of the reaction time on a $[4+1]$ cyclative capture access to indolizines

1.6. Table 6 . The effect of the reaction temperature on a $[4+1]$ cyclative capture access to indolizines

1.7. Table 6. The effect of molar ratio (1a:2a) on a $[4+1]$ cyclative capture access to indolizines

2. General Procedure for the Synthesis for Pyrimido[1,6-a]indole-1(2H)-Ones Derivatives. ..5

3. Controlled Experiments for Mechanism Studies.

4. Single Crystal Data for $3 \mathrm{~s}$

5. References.

6. ${ }^{1} \mathrm{H}$ NMR and ${ }^{13} \mathrm{C}$ NMR Spectrum for All Isolated Products. .42 


\section{General experimental information}

All reactions were carried out in flame-dried sealed tubes with magnetic stirring. Unless otherwise noted, all experiments were performed under argon atmosphere. All reagents were purchased from TCI, Acros or Strem. Solvents were treated with $4 \AA$ molecular sieves or sodium and distilled prior to use. Catalysts $\left[\mathrm{Cp} * \mathrm{Co}(\mathrm{CO}) \mathrm{I}_{2}\right]^{[1]}$ and $\left[\mathrm{Cp} * \mathrm{Co}\left(\mathrm{CH}_{3} \mathrm{CN}\right)_{3}\right]\left[\mathrm{SbF}_{6}\right]_{2}{ }^{[2]}$ were prepared according to the reported procedure. The starting materials 2-phenylpyridine substrates $\mathbf{1 a - 1} \mathbf{w}^{[3]}$ and 2-vinylpyridine substrates $\mathbf{1 x}-\mathbf{1 z},{ }^{[4]} \mathbf{1 a z}-\mathbf{1 i z}{ }^{[4]}$ were prepared according to the previously reported procedures. Purifications of reaction products were carried out by flash chromatography using Qingdao Haiyang Chemical Co. Ltd silica gel (40-63 mm). Infrared spectra (IR) were recorded on a Brucker TENSOR 27 FTIR spectrophotometer and are reported as wavelength numbers $\left(\mathrm{cm}^{-1}\right)$. Infrared spectra were recorded by preparing a $\mathrm{KBr}$ pellet containing the title compound. ${ }^{1} \mathrm{H}$ NMR and ${ }^{13} \mathrm{C}$ NMR spectra were recorded with tetramethylsilane (TMS) as internal standard at ambient temperature unless otherwise indicated on a Bruker Avance DPX 600 fourier Transform spectrometer operating at $400 \mathrm{MHz}$ for ${ }^{1} \mathrm{H}$ NMR and $100 \mathrm{MHz}$ for ${ }^{13} \mathrm{C}$ NMR. Chemical shifts are reported in parts per million (ppm) and coupling constants are reported as Hertz (Hz). Splitting patterns are designated as singlet (s), broad singlet (bs), doublet (d), triplet (t). Splitting patterns that could not be interpreted or easily visualized are designated as multiple $(\mathrm{m})$. Low resolution mass spectra were recorded using a Waters HPLC/ZQ4000 Mass Spectrometer. High resolution mass spectra (HRMS) were recorded on an IF-TOF spectrometer (Micromass). Gas chromatograph mass spectra were obtained with a SHIMADZU model GCMS-QP5000 spectrometer. Crystal data were collected on a Bruker D8 Advance employing graphite monochromated Mo - Ka radiation $(\lambda=0.71073 \AA)$ at 293 (2) $\mathrm{K}$ and operating in the $\varphi$ - $\omega$ scan mode. The structure was solved by direct methods SHELXS-97. 
1.1. Table 1. Catalyst screening for a $[4+1]$ cyclative capture access to indolizines ${ }^{a}$

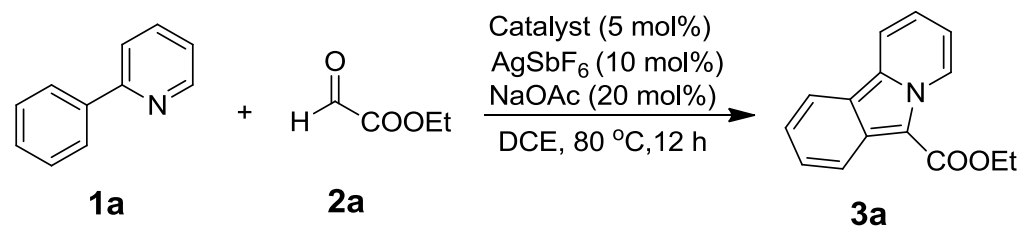

\begin{tabular}{ccc}
\hline Entry & catalyst & yield $(\%)^{b}$ \\
\hline 1 & $\mathrm{Co}(\mathrm{acac})_{2}$ & 0 \\
2 & $\mathrm{Co}(\mathrm{acac})_{3}$ & 0 \\
3 & $\mathrm{CoCl}_{2}$ & 0 \\
4 & $\mathrm{CoI}_{2}$ & 0 \\
5 & $\left.\mathrm{Cp} \mathrm{Co}_{2} \mathrm{CO}\right) \mathrm{I}_{2}$ & 28 \\
6 & {$\left[\mathrm{Cp} * \mathrm{Co}(\mathrm{MeCN})_{3}\right]\left[\mathrm{SbF}_{6}\right]_{2}$} & $21^{\mathrm{c}}$ \\
\hline
\end{tabular}

${ }^{a}$ All the reactions were carried out using 2-phenylpyridine $\mathbf{1 a}(0.2 \mathrm{mmol})$, ethyl glyoxylate $\mathbf{2 a}(0.2$ mmol), catalyst $(5 \mathrm{~mol} \%), \mathrm{AgSbF}_{6}(10 \mathrm{~mol} \%)$ and $\mathrm{NaOAc}(20 \mathrm{~mol} \%)$ in 1, 2-dichloroethane (DCE) $(2.0 \mathrm{~mL})$ at $80{ }^{\circ} \mathrm{C}$ for $12 \mathrm{~h}$ in a sealed reaction tube, followed by flash chromatography on $\mathrm{SiO}_{2} \cdot{ }^{b}$ Isolated yield. ${ }^{\mathrm{c}} \mathrm{No} \mathrm{AgSbF}_{6}$.

\subsection{Table 2. The effect of Ag salts on a $[4+1]$ cyclative capture access to indolizines ${ }^{a}$}

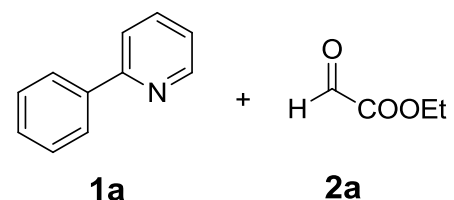

$1 \mathbf{a}$

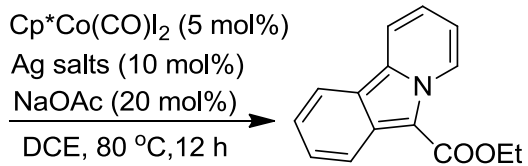

$3 a$

\begin{tabular}{ccc}
\hline entry & Ag salts & yield $(\%)^{b}$ \\
\hline 1 & $\mathrm{AgSbF}_{6}$ & 28 \\
2 & $\mathrm{AgBF}_{4}$ & 19 \\
3 & $\mathrm{AgNTf}_{2}$ & 25 \\
4 & $\mathrm{AgOAc}^{b}$ & 14 \\
5 & $\mathrm{AgClO}_{4}$ & 0 \\
\hline
\end{tabular}

${ }^{a}$ All the reactions were carried out using 2-phenylpyridine 1a $(0.2 \mathrm{mmol})$, ethyl glyoxylate $\mathbf{2 a}(0.2$ $\mathrm{mmol}), \mathrm{Cp} * \mathrm{Co}(\mathrm{CO}) \mathrm{I}_{2}(5 \mathrm{~mol} \%), \mathrm{Ag}$ salts $(10 \mathrm{~mol} \%)$ and $\mathrm{NaOAc}(20 \mathrm{~mol} \%)$ in $1,2-$ dichloroethane (DCE) $(2.0 \mathrm{~mL})$ at $80{ }^{\circ} \mathrm{C}$ for $12 \mathrm{~h}$ in a sealed reaction tube, followed by flash chromatography on $\mathrm{SiO}_{2} \cdot{ }^{b}$ Isolated yield.

\subsection{Table 3. The effect of additive on a $[4+1]$ cyclative capture access to indolizines ${ }^{a}$}

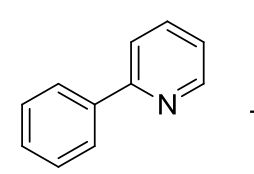

$1 \mathrm{a}$<smiles>CCOC(=O)C=O</smiles>

$2 a$

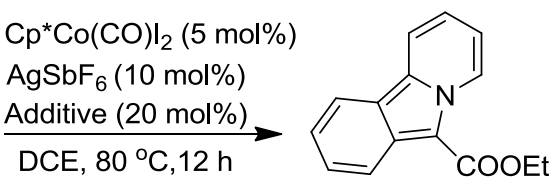

$3 a$

\begin{tabular}{ccc}
\hline entry & additive & yield $(\%)^{b}$ \\
\hline 1 & NaOAc & 28 \\
2 & KOAc & 25 \\
3 & CsOAc & 24 \\
4 & $\mathrm{AgOAc}$ & 16 \\
5 & $\mathrm{Cu}(\mathrm{OAc})_{2}$ & 47 \\
6 & $\mathrm{AcOH}$ & 18 \\
7 & PivOH & 22 \\
\hline
\end{tabular}

\footnotetext{
${ }^{a}$ All the reactions were carried out using 2-phenylpyridine 1a $(0.2 \mathrm{mmol})$, ethyl glyoxylate $\mathbf{2 a}(0.2$
} 
mmol), $\mathrm{Cp} * \mathrm{Co}(\mathrm{CO}) \mathrm{I}_{2}(5 \mathrm{~mol} \%), \mathrm{AgSbF}_{6}(10 \mathrm{~mol} \%)$ and additive $(20 \mathrm{~mol} \%)$ in $1,2-$ dichloroethane (DCE) $(2.0 \mathrm{~mL})$ at $80^{\circ} \mathrm{C}$ for $12 \mathrm{~h}$ in a sealed reaction tube, followed by flash chromatography on $\mathrm{SiO}_{2} \cdot{ }^{b}$ Isolated yield.

\subsection{Table 4. The effect of solvents on a $[4+1]$ cyclative capture access to indolizines ${ }^{a}$}

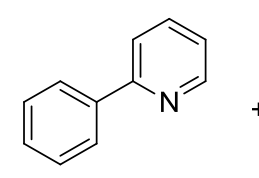

$1 \mathrm{a}$<smiles>CCOC(=O)C=O</smiles>

$2 a$

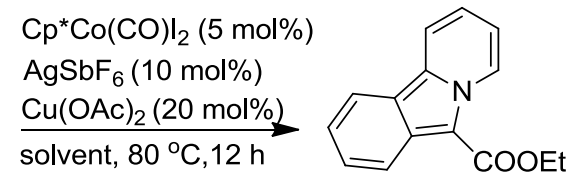

$3 a$

\begin{tabular}{ccc}
\hline entry & solvent & yield $(\%)^{b}$ \\
\hline 1 & DCE & 47 \\
2 & THF & 36 \\
3 & toluene & 18 \\
4 & DMF & trace \\
5 & MeCN & 29 \\
6 & 1,6 -dioxane & 22 \\
\hline
\end{tabular}

${ }^{a}$ All the reactions were carried out using 2-phenylpyridine 1a $(0.2 \mathrm{mmol})$, ethyl glyoxylate $\mathbf{2 a}(0.2$ $\mathrm{mmol}), \mathrm{Cp}^{*} \mathrm{Co}(\mathrm{CO}) \mathrm{I}_{2}\left(5 \mathrm{~mol}^{2}\right), \mathrm{AgSbF}_{6}(10 \mathrm{~mol} \%)$ and $\mathrm{Cu}(\mathrm{OAc})_{2}(20 \mathrm{~mol} \%)$ in solvent $(2.0 \mathrm{~mL})$ at $80{ }^{\circ} \mathrm{C}$ for $12 \mathrm{~h}$ in a sealed reaction tube, followed by flash chromatography on $\mathrm{SiO}_{2} \cdot{ }^{b}$ Isolated yield.

\subsection{Table 5. The effect of the reaction time on a $[4+1]$ cyclative capture access to indolizines}

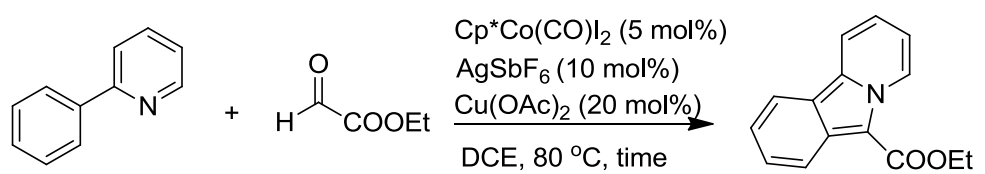

$1 \mathrm{a}$ 2a
$3 a$

\begin{tabular}{ccc}
\hline entry & time $(\mathrm{h})$ & yield $(\%)^{b}$ \\
\hline 1 & 6 & 41 \\
2 & 12 & 47 \\
3 & 24 & 54 \\
4 & 36 & 53 \\
\hline
\end{tabular}

${ }^{a}$ All the reactions were carried out using 2-phenylpyridine 1a $(0.2 \mathrm{mmol})$, ethyl glyoxylate $\mathbf{2 a}(0.2$ $\mathrm{mmol}), \mathrm{Cp}^{*} \mathrm{Co}(\mathrm{CO}) \mathrm{I}_{2}(5 \mathrm{~mol} \%), \mathrm{AgSbF}_{6}(10 \mathrm{~mol} \%)$ and $\mathrm{Cu}(\mathrm{OAc})_{2}(20 \mathrm{~mol} \%)$ in 1 , 2 dichloroethane (DCE) $(2.0 \mathrm{~mL})$ at $80{ }^{\circ} \mathrm{C}$ for the given time in a sealed reaction tube, followed by flash chromatography on $\mathrm{SiO}_{2}{ }^{b}$ Isolated yield.

1.6. Table 6. The effect of the reaction temperature on a $[4+1]$ cyclative capture access to indolizines $^{a}$

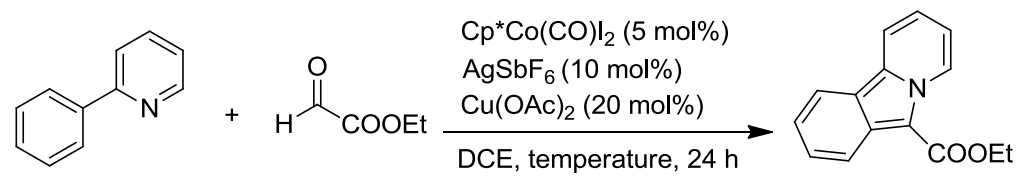

1a

$2 a$

$3 a$

\begin{tabular}{ccc}
\hline entry & temp. $\left({ }^{\circ} \mathrm{C}\right)$ & yield $(\%)^{b}$ \\
\hline 1 & 60 & 18 \\
2 & 80 & 54 \\
3 & 100 & 65 \\
4 & 110 & 71
\end{tabular}


${ }^{a}$ All the reactions were carried out using 2-phenylpyridine 1a $(0.2 \mathrm{mmol})$, ethyl glyoxylate $\mathbf{2 a}(0.2$ mmol), $\mathrm{Cp} * \mathrm{Co}(\mathrm{CO}) \mathrm{I}_{2}(5 \mathrm{~mol} \%), \mathrm{AgSbF}_{6}(10 \mathrm{~mol} \%)$ and $\mathrm{Cu}(\mathrm{OAc})_{2}(20 \mathrm{~mol} \%)$ in $1,2-$ dichloroethane (DCE) $(2.0 \mathrm{~mL})$ at the given temperature for $24 \mathrm{~h}$ in a sealed reaction tube, followed by flash chromatography on $\mathrm{SiO}_{2} .{ }^{b}$ Isolated yield.

1.7. Table 7. The effect of molar ratio $(1 \mathrm{a}: 2 \mathrm{a})$ on a $[4+1]$ cyclative capture access to indolizines $^{a}$

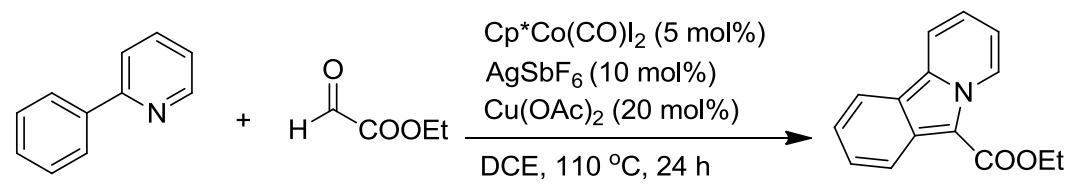

$1 \mathrm{a}$

2a

3a

\begin{tabular}{ccc}
\hline entry & molar ratio (1a:2a) & yield (\%) ${ }^{b}$ \\
\hline 1 & $1: 1$ & 71 \\
2 & $1: 1.5$ & 77 \\
3 & $1: 2$ & 88 \\
4 & $1: 3$ & 84 \\
5 & $2: 1$ & 46 \\
\hline
\end{tabular}

${ }^{a}$ All the reactions were carried out using 2-phenylpyridine 1a $(0.2 \mathrm{mmol})$, ethyl glyoxylate 2a $(\mathrm{x}$ mmol) at the given molar ratio, $\mathrm{Cp} * \mathrm{Co}(\mathrm{CO}) \mathrm{I}_{2}(5 \mathrm{~mol} \%), \mathrm{AgSbF}_{6}(10 \mathrm{~mol} \%)$ and $\mathrm{Cu}(\mathrm{OAc})_{2}(20$ mol\%) in 1, 2-dichloroethane (DCE) $(2.0 \mathrm{~mL})$ at $110{ }^{\circ} \mathrm{C}$ for $24 \mathrm{~h}$ in a sealed reaction tube, followed by flash chromatography on $\mathrm{SiO}_{2}{ }^{b}$ Isolated yield.

\section{General procedure for the synthesis for indolizines derivatives (3a-3kz)}

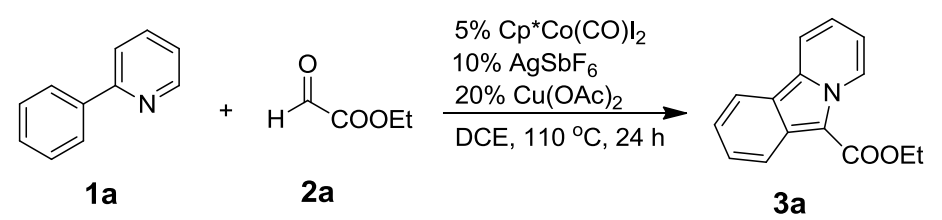

A $10 \mathrm{~mL}$ of reaction tube was charged with 2-phenylpyridine $\mathbf{1}(0.2 \mathrm{mmol}), \mathrm{Cp} * \mathrm{Co}(\mathrm{CO}) \mathrm{I}_{2}(4.8 \mathrm{mg}$, $5 \mathrm{~mol} \%), \mathrm{AgSbF}_{6}(6.8 \mathrm{mg}, 10 \mathrm{~mol} \%), \mathrm{Cu}(\mathrm{OAc})_{2}(7.2 \mathrm{mg}, 20 \mathrm{~mol} \%)$ and DCE (1.5 mL) under Ar atmosphere. Then ethyl glyoxylate $2(0.4 \mathrm{mmol})$ in DCE $(0.5 \mathrm{~mL})$ was added in one-pot under $\mathrm{Ar}$ and the mixture was stirred at $110{ }^{\circ} \mathrm{C}$ for $24 \mathrm{~h}$. The corresponding reaction mixture was filtered through a pad of Celite, washed with EtOAc and concentrated under reduced pressure. The residue was purified by flash chromatography on silical gel using dichloromethane/petroleum ether as eluent to afford the desired products 3 .

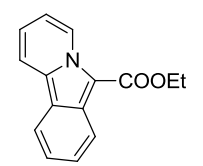

$3 \mathbf{a}$

Ethyl pyrido[2,1-a]isoindole-6-carboxylate (3a) ${ }^{[5]}$ : yellow solid; $42.1 \mathrm{mg}, 88 \%$ yield; m.p. 65.3-66.2 ${ }^{\circ} \mathrm{C} .{ }^{1} \mathrm{H}$ NMR $\left(400 \mathrm{MHz}, \mathrm{CDCl}_{3}\right) \delta 9.99(\mathrm{~d}, J=6.9 \mathrm{~Hz}, 1 \mathrm{H}), 8.28(\mathrm{~d}, J=8.5 \mathrm{~Hz}, 1 \mathrm{H}), 8.14-$ $8.08(\mathrm{~m}, 2 \mathrm{H}), 7.58-7.52(\mathrm{~m}, 1 \mathrm{H}), 7.33-7.25(\mathrm{~m}, 2 \mathrm{H}), 7.22(\mathrm{dd}, J=6.9,6.0 \mathrm{~Hz}, 1 \mathrm{H}), 4.51(\mathrm{q}, J=7.1$ $\mathrm{Hz}, 2 \mathrm{H}), 1.52(\mathrm{t}, J=7.1 \mathrm{~Hz}, 3 \mathrm{H}) ;{ }^{13} \mathrm{C}$ NMR $\left(101 \mathrm{MHz}, \mathrm{CDCl}_{3}\right) \delta 162.5,132.7,130.9,128.1,127.5$, $121.5,120.7,119.8,119.4,118.6,117.4,117.2,104.0,59.5,14.8 ; \mathrm{MS}(\mathrm{ESI}): \mathrm{m} / \mathrm{z}=239.13[\mathrm{M}]^{+} ; \mathrm{IR}$ 
(KBr): 2977, 1665, 1439, 1322, 1185, 1109, $1044 \mathrm{~cm}^{-1}$.

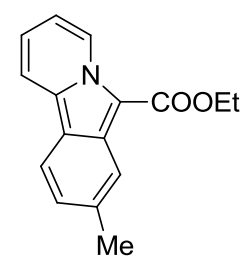

$3 b$

Ethyl 8-methylpyrido[2,1-a]isoindole-6-carboxylate (3b) ${ }^{[5]}$ : yellow solid; $42.5 \mathrm{mg}$, $84 \%$ yield; m.p. 69.7-71.1 ${ }^{\circ} \mathrm{C} .{ }^{1} \mathrm{H}$ NMR $\left(400 \mathrm{MHz}, \mathrm{CDCl}_{3}\right) \delta 9.96(\mathrm{~d}, J=6.8 \mathrm{~Hz}, 1 \mathrm{H}), 8.08(\mathrm{~d}, J=8.5 \mathrm{~Hz}, 1 \mathrm{H})$, 8.05 (s, 1H), 7.99 (d, $J=8.3 \mathrm{~Hz}, 1 \mathrm{H}), 7.30-7.26(\mathrm{~m}, 1 \mathrm{H}), 7.21-7.16(\mathrm{~m}, 1 \mathrm{H}), 7.11$ (d, $J=8.3 \mathrm{~Hz}$, $1 \mathrm{H}), 4.52(\mathrm{q}, J=7.1 \mathrm{~Hz}, 2 \mathrm{H}), 2.57$ (s, 3H), $1.53(\mathrm{t}, J=7.1 \mathrm{~Hz}, 3 \mathrm{H}) ;{ }^{13} \mathrm{C}$ NMR $\left(101 \mathrm{MHz}, \mathrm{CDCl}_{3}\right) \delta$ 162.6, 138.2, 132.9, 131.5, 127.7, 123.1, 121.6, 119.1, 118.8, 117.1, 117.0, 116.8, 103.6, 59.4, 22.6, 14.9; MS (ESI): $\mathrm{m} / \mathrm{z}=253.11[\mathrm{M}]^{+}$; IR (KBr): 2979, 1657, 1440, 1324, 1176, 1113, $1043 \mathrm{~cm}^{-1}$.

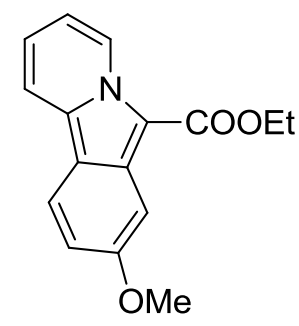

$3 c$

Ethyl 8-methoxypyrido[2,1-a]isoindole-6-carboxylate (3c) ${ }^{[5]}$ : yellow solid; $36.0 \mathrm{mg}$, 67\% yield; m.p. 95.1-96.2 ${ }^{\circ} \mathrm{C} .{ }^{1} \mathrm{H}$ NMR $\left(400 \mathrm{MHz}, \mathrm{CDCl}_{3}\right) \delta 9.89(\mathrm{~d}, J=4.6 \mathrm{~Hz}, 1 \mathrm{H}), 7.97(\mathrm{dd}, J=16.6,8.7 \mathrm{~Hz}$, 2H), $7.61(\mathrm{~s}, 1 \mathrm{H}), 7.28-7.23(\mathrm{~m}, 1 \mathrm{H}), 7.13(\mathrm{t}, J=6.9 \mathrm{~Hz}, 1 \mathrm{H}), 6.91(\mathrm{dd}, J=8.9,2.1 \mathrm{~Hz}, 1 \mathrm{H}), 4.50(\mathrm{q}$, $J=7.1 \mathrm{~Hz}, 2 \mathrm{H}), 3.96(\mathrm{~s}, 3 \mathrm{H}), 1.52(\mathrm{t}, J=7.1 \mathrm{~Hz}, 3 \mathrm{H}) ;{ }^{13} \mathrm{C} \mathrm{NMR}\left(101 \mathrm{MHz}, \mathrm{CDCl}_{3}\right) \delta 162.5,160.3$, 133.0, 132.7, 127.8, 122.1, 120.8, 116.6, 116.0, 113.7, 113.6, 103.8, 98.7, 59.3, 55.2, 14.8; MS (ESI): $\mathrm{m} / \mathrm{z}=269.08[\mathrm{M}]^{+} ; \mathrm{IR}(\mathrm{KBr}): 2976,1655,1438,1326,1182,1112,1042 \mathrm{~cm}^{-1}$.<smiles>CCOC(=O)c1c2cc(-c3ccccc3)ccc2c2ccccn12</smiles>

3d

Ethyl 8-phenylpyrido[2,1-a]isoindole-6-carboxylate (3d) ${ }^{[5]}$ : yellow solid; $49.7 \mathrm{mg}$, 79\% yield; m.p. 108.4-109.3 ${ }^{\circ} \mathrm{C} .{ }^{1} \mathrm{H}$ NMR $\left(400 \mathrm{MHz}, \mathrm{CDCl}_{3}\right) \delta 9.99(\mathrm{~d}, J=6.9 \mathrm{~Hz}, 1 \mathrm{H}), 8.50(\mathrm{~s}, 1 \mathrm{H}), 8.13$ (t, $J=$ $8.2 \mathrm{~Hz}, 2 \mathrm{H}), 7.76(\mathrm{~d}, J=7.4 \mathrm{~Hz}, 2 \mathrm{H}), 7.55-7.47(\mathrm{~m}, 3 \mathrm{H}), 7.39(\mathrm{t}, J=7.3 \mathrm{~Hz}, 1 \mathrm{H}), 7.30(\mathrm{t}, J=7.7 \mathrm{~Hz}$, $1 \mathrm{H}), 7.22(\mathrm{dd}, J=6.9,6.0 \mathrm{~Hz}, 1 \mathrm{H}), 4.53(\mathrm{q}, J=7.1 \mathrm{~Hz}, 2 \mathrm{H}), 1.53(\mathrm{t}, J=7.1 \mathrm{~Hz}, 3 \mathrm{H}) ;{ }^{13} \mathrm{C}$ NMR $(101$ $\left.\mathrm{MHz}, \mathrm{CDCl}_{3}\right) \delta 162.5,142.2,141.0,132.7,131.4,128.8,127.6,127.2,121.8,120.8,119.8,118.0$, 
117.8, 117.5, 117.2, 104.3, 59.5, 14.8; MS (ESI): $\mathrm{m} / \mathrm{z}=315.07[\mathrm{M}]^{+}$; IR (KBr): 2977, 1660, 1446, $1327,1186,1114,1044 \mathrm{~cm}^{-1}$.

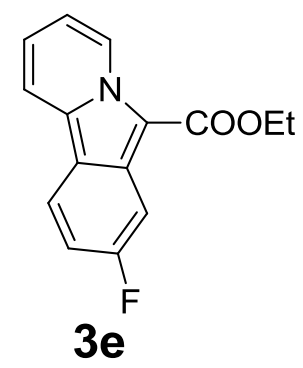

Ethyl 8-fluoropyrido[2,1-a]isoindole-6-carboxylate (3e) : yellow solid; $38.0 \mathrm{mg}$, $74 \%$ yield; m.p. 84.7-85.5 ${ }^{\circ} \mathrm{C} .{ }^{1} \mathrm{H}$ NMR $\left(400 \mathrm{MHz}, \mathrm{CDCl}_{3}\right) \delta 9.91(\mathrm{~d}, J=6.7 \mathrm{~Hz}, 1 \mathrm{H}), 8.03-7.94(\mathrm{~m}, 2 \mathrm{H}), 7.79$ $(\mathrm{dd}, J=10.8,1.6 \mathrm{~Hz}, 1 \mathrm{H}), 7.28(\mathrm{dd}, J=14.5,6.3 \mathrm{~Hz}, 1 \mathrm{H}), 7.18(\mathrm{t}, J=6.9 \mathrm{~Hz}, 1 \mathrm{H}), 6.98(\mathrm{td}, J=8.9$, $2.2 \mathrm{~Hz}, 1 \mathrm{H}), 4.48(\mathrm{q}, J=7.1 \mathrm{~Hz}, 2 \mathrm{H}), 1.51(\mathrm{t}, J=7.1 \mathrm{~Hz}, 3 \mathrm{H}) ;{ }^{13} \mathrm{C} \mathrm{NMR}\left(101 \mathrm{MHz}, \mathrm{CDCl}_{3}\right) \delta 163.1(\mathrm{~d}$, $\mathrm{J}=244.4 \mathrm{~Hz}), 162.2,132.6,131.7(\mathrm{~d}, \mathrm{~J}=12.1 \mathrm{~Hz}), 127.6,122.3,121.4(\mathrm{~d}, \mathrm{~J}=11.1 \mathrm{~Hz}), 117.0,116.9$, 115.3, $110.8(\mathrm{~d}, \mathrm{~J}=27.3 \mathrm{~Hz}), 103.9(\mathrm{~d}, \mathrm{~J}=25.3 \mathrm{~Hz}), 77.3,77.0,76.7,59.6,14.8$; HR-MS (ESI) calcd for $[\mathrm{M}+1]^{+}: \mathrm{C}_{15} \mathrm{H}_{12} \mathrm{FNO}_{2}$ : 258.0925, found: 258.0928; IR (KBr): 2978, 1659, 1441, 1323, 1176, $1118,1049 \mathrm{~cm}^{-1}$.<smiles>CCOC(=O)c1c2cc(Cl)ccc2c2ccccn12</smiles>

Ethyl 8-chloropyrido[2,1-a]isoindole-6-carboxylate (3f) ${ }^{[5]}$ : yellow solid; $43.1 \mathrm{mg}, 79 \%$ yield; m.p. 93.7-94.8 ${ }^{\circ} \mathrm{C} .{ }^{1} \mathrm{H}$ NMR $\left(400 \mathrm{MHz}, \mathrm{CDCl}_{3}\right) \delta 9.92(\mathrm{~d}, J=6.9 \mathrm{~Hz}, 1 \mathrm{H}), 8.17$ (s, 1H), 8.03 (d, $J=$ $8.5 \mathrm{~Hz}, 1 \mathrm{H}), 7.94(\mathrm{~d}, J=8.6 \mathrm{~Hz}, 1 \mathrm{H}), 7.30$ (t, $J=7.7 \mathrm{~Hz}, 1 \mathrm{H}), 7.24-7.19(\mathrm{~m}, 1 \mathrm{H}), 7.16$ (dd, $J=8.6$, $1.8 \mathrm{~Hz}, 1 \mathrm{H}), 4.50(\mathrm{q}, J=7.1 \mathrm{~Hz}, 2 \mathrm{H}), 1.52(\mathrm{t}, J=7.1 \mathrm{~Hz}, 3 \mathrm{H}) ;{ }^{13} \mathrm{C} \mathrm{NMR}\left(101 \mathrm{MHz}, \mathrm{CDCl}_{3}\right) \delta 162.1$, 134.1, 132.4, 131.3, 127.6, 122.2, 121.5, 120.7, 118.9, 117.4, 117.3, 116.6, 103.6, 59.6, 14.8; MS (ESI): $\mathrm{m} / \mathrm{z}=273.02[\mathrm{M}]^{+} ; \mathrm{IR}(\mathrm{KBr}): 2977,1660,1446,1327,1186,1114,1044 \mathrm{~cm}^{-1}$.<smiles></smiles> 
Ethyl 8-acetylpyrido[2,1-a]isoindole-6-carboxylate (3g) : yellow solid; $50.0 \mathrm{mg}$, 89\% yield; m.p. 112.4-113.3 ${ }^{\circ} \mathrm{C} .{ }^{1} \mathrm{H}$ NMR $\left(400 \mathrm{MHz}, \mathrm{CDCl}_{3}\right) \delta 9.90(\mathrm{~d}, J=6.9 \mathrm{~Hz}, 1 \mathrm{H}), 8.84(\mathrm{~s}, 1 \mathrm{H}), 8.09(\mathrm{~d}, J=8.4$ $\mathrm{Hz}, 1 \mathrm{H}), 8.04(\mathrm{dd}, J=8.6,0.6 \mathrm{~Hz}, 1 \mathrm{H}), 7.33-7.28(\mathrm{~m}, 1 \mathrm{H}), 7.24(\mathrm{td}, J=6.9,1.5 \mathrm{~Hz}, 1 \mathrm{H}), 4.52(\mathrm{q}, J=$ $7.1 \mathrm{~Hz}, 2 \mathrm{H}), 2.73(\mathrm{~s}, 3 \mathrm{H}), 1.56(\mathrm{t}, J=7.1 \mathrm{~Hz}, 3 \mathrm{H}) ;{ }^{13} \mathrm{C} \mathrm{NMR}\left(101 \mathrm{MHz}, \mathrm{CDCl}_{3}\right) \delta 198.6,162.0,136.3$, 132.0, 129.8, 128.9, 127.5, 127.3, 122.5, 121.9, 120.1, 119.5, 118.9, 118.1, 118.0, 105.0, 59.8, 26.6, 14.7; HR-MS (ESI) calcd for $[\mathrm{M}+1]^{+}: \mathrm{C}_{17} \mathrm{H}_{15} \mathrm{NO}_{3}: 282.1125$, found: 282.1131; IR (KBr): 2975 , $1722,1663,1439,1322,1176,1108,1041 \mathrm{~cm}^{-1}$.

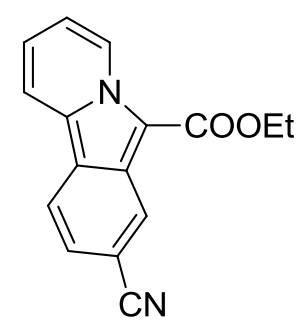

\section{$3 \mathrm{~h}$}

Ethyl 8-cyanopyrido[2,1-a]isoindole-6-carboxylate (3h) : yellow solid; $44.9 \mathrm{mg}, 85 \%$ yield; m.p. 138.6-140.3 ${ }^{\circ} \mathrm{C} .{ }^{1} \mathrm{H}$ NMR (400 MHz, $\left.\mathrm{CDCl}_{3}\right) \delta 9.95(\mathrm{~d}, J=6.9 \mathrm{~Hz}, 1 \mathrm{H}), 8.54(\mathrm{~s}, 1 \mathrm{H}), 8.11(\mathrm{dd}, J$ $=18.1,8.4 \mathrm{~Hz}, 2 \mathrm{H}), 7.40(\mathrm{t}, J=7.6 \mathrm{~Hz}, 1 \mathrm{H}), 7.33(\mathrm{t}, J=8.2 \mathrm{~Hz}, 2 \mathrm{H}), 4.52(\mathrm{q}, J=7.1 \mathrm{~Hz}, 2 \mathrm{H}), 1.53(\mathrm{t}$, $J=7.1 \mathrm{~Hz}, 3 \mathrm{H}) ;{ }^{13} \mathrm{C} \mathrm{NMR}\left(101 \mathrm{MHz}, \mathrm{CDCl}_{3}\right) \delta 161.7,131.9,128.9,127.6,126.1,122.6,121.3,120.5$, 120.1, 119.1, 118.6, 118.1, 110.8, 104.4, 60.0, 14.8; HR-MS (ESI) calcd for $[\mathrm{M}+1]^{+}: \mathrm{C}_{16} \mathrm{H}_{12} \mathrm{~N}_{2} \mathrm{O}_{2}$ : 265.0972, found: 265.0976; IR (KBr): 2978, 2235, 1658, 1445, 1329, 1188, 1112, $1038 \mathrm{~cm}^{-1}$.

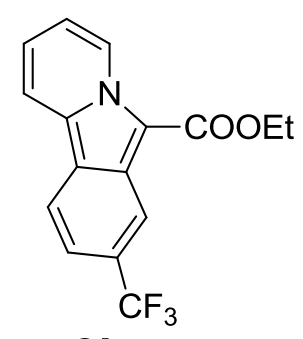

$3 \mathbf{i}$

Ethyl 8-(trifluoromethyl)pyrido[2,1-a]isoindole-6-carboxylate $(3 \mathrm{3i}){ }^{[5]}$ : yellow solid; $55.2 \mathrm{mg}$, 90\% yield; m.p. $129.1-129.9{ }^{\circ} \mathrm{C} .{ }^{1} \mathrm{H}$ NMR $\left(400 \mathrm{MHz}, \mathrm{CDCl}_{3}\right) \delta 9.98(\mathrm{~d}, J=6.9 \mathrm{~Hz}, 1 \mathrm{H}), 8.55(\mathrm{~s}, 1 \mathrm{H})$, $8.14(\mathrm{~d}, J=8.5 \mathrm{~Hz}, 2 \mathrm{H}), 7.41(\mathrm{~d}, J=8.6 \mathrm{~Hz}, 1 \mathrm{H}), 7.36(\mathrm{t}, J=7.7 \mathrm{~Hz}, 1 \mathrm{H}), 7.29(\mathrm{t}, J=6.9 \mathrm{~Hz}, 1 \mathrm{H})$, $4.53(\mathrm{~d}, J=7.1 \mathrm{~Hz}, 2 \mathrm{H}), 1.54(\mathrm{t}, J=7.1 \mathrm{~Hz}, 3 \mathrm{H}) ;{ }^{13} \mathrm{C} \mathrm{NMR}\left(101 \mathrm{MHz}, \mathrm{CDCl}_{3}\right) \delta 162.0,130.7(\mathrm{~d}, \mathrm{~J}=$ $276.7 \mathrm{~Hz}), 129.6(\mathrm{~d}, \mathrm{~J}=31.3 \mathrm{~Hz}), 127.6,126.1,123.4,122.2,120.3,119.3,118.1(\mathrm{~d}, \mathrm{~J}=21.2 \mathrm{~Hz})$, $117.8(\mathrm{~d}, \mathrm{~J}=5.1 \mathrm{~Hz}), 116.5(\mathrm{~d}, \mathrm{~J}=3.0 \mathrm{~Hz}), 104.8,59.9,14.6 ; \mathrm{MS}(\mathrm{ESI}): \mathrm{m} / \mathrm{z}=307.03[\mathrm{M}]^{+}$; IR (KBr): 2979, 1676, 1455, 1313, 1189, 1115, $1058 \mathrm{~cm}^{-1}$. 


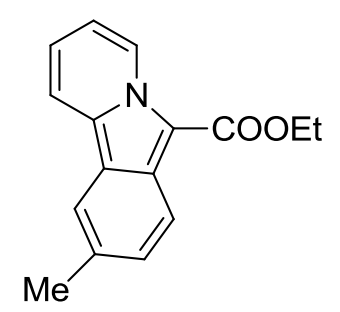

3j

Ethyl 9-methylpyrido[2,1-a]isoindole-6-carboxylate (3j) ${ }^{[5]}$ : yellow solid; $44.0 \mathrm{mg}, 87 \%$ yield; m.p. 80.5-81.6 ${ }^{\circ} \mathrm{C} .{ }^{1} \mathrm{H}$ NMR $\left(400 \mathrm{MHz}, \mathrm{CDCl}_{3}\right) \delta 9.94(\mathrm{~d}, J=6.8 \mathrm{~Hz}, 1 \mathrm{H}), 8.17(\mathrm{~d}, J=8.6 \mathrm{~Hz}, 1 \mathrm{H})$, $8.07(\mathrm{~d}, J=8.4 \mathrm{~Hz}, 1 \mathrm{H}), 7.86(\mathrm{~s}, 1 \mathrm{H}), 7.38(\mathrm{~d}, J=8.6 \mathrm{~Hz}, 1 \mathrm{H}), 7.25(\mathrm{t}, J=7.6 \mathrm{~Hz}, 1 \mathrm{H}), 7.18$ (td, $J=$ 6.9, $1.2 \mathrm{~Hz}, 1 \mathrm{H}), 4.50(\mathrm{q}, J=7.1 \mathrm{~Hz}, 2 \mathrm{H}), 2.54(\mathrm{~s}, 3 \mathrm{H}), 1.51(\mathrm{t}, J=7.1 \mathrm{~Hz}, 3 \mathrm{H}) ;{ }^{13} \mathrm{C}$ NMR $(101 \mathrm{MHz}$, $\left.\mathrm{CDCl}_{3}\right) \delta 162.5,132.2,130.5,130.2,129.3,127.4,121.1,119.6,118.9,118.3,117.4,117.0,103.8,59.4$, 21.7, 14.8; MS (ESI): $\mathrm{m} / \mathrm{z}=253.09[\mathrm{M}]^{+}$; IR (KBr): 2977, 1660, 1432, 1309, 1115, $1047 \mathrm{~cm}^{-1}$.<smiles>CCOC(=O)c1c2ccc(OC)cc2c2ccccn12</smiles>

3k

Ethyl 9-methoxypyrido[2,1-a]isoindole-6-carboxylate (3k) : yellow solid; $39.3 \mathrm{mg}$, $73 \%$ yield; m.p. 97.8-99.4 ${ }^{\circ} \mathrm{C} .{ }^{1} \mathrm{H}$ NMR $\left(400 \mathrm{MHz}, \mathrm{CDCl}_{3}\right) \delta 9.90(\mathrm{~d}, J=6.7 \mathrm{~Hz}, 1 \mathrm{H}), 8.20(\mathrm{~d}, J=9.1 \mathrm{~Hz}, 1 \mathrm{H})$, $8.05(\mathrm{~d}, J=8.4 \mathrm{~Hz}, 1 \mathrm{H}), 7.40(\mathrm{~d}, J=1.7 \mathrm{~Hz}, 1 \mathrm{H}), 7.27-7.21(\mathrm{~m}, 2 \mathrm{H}), 7.16(\mathrm{t}, J=6.4 \mathrm{~Hz}, 1 \mathrm{H}), 4.50(\mathrm{q}$, $J=7.1 \mathrm{~Hz}, 2 \mathrm{H}), 3.94(\mathrm{~s}, 3 \mathrm{H}), 1.51(\mathrm{t}, J=7.1 \mathrm{~Hz}, 3 \mathrm{H}) ;{ }^{13} \mathrm{C} \mathrm{NMR}\left(101 \mathrm{MHz}, \mathrm{CDCl}_{3}\right) \delta 162.3,154.7$, 131.7, 127.4, 126.5, 121.4, 120.9, 120.5, 118.8, 117.4, 116.7, 103.8, 98.2, 59.4, 55.5, 14.8; HR-MS (ESI) calcd for $[\mathrm{M}+1]^{+}: \mathrm{C}_{16} \mathrm{H}_{15} \mathrm{NO}_{3}: 270.1125$, found: 270.1127; IR (KBr): 2978, 1661, 1442 , $1323,1180,1110,1045 \mathrm{~cm}^{-1}$.<smiles>CCOC(=O)c1c2ccc(Cl)cc2c2ccccn12</smiles>

3I

Ethyl 9-chloropyrido[2,1-a]isoindole-6-carboxylate (3I) ${ }^{[5]}$ : yellow solid; $46.9 \mathrm{mg}, 86 \%$ yield; m.p. 88.8-90.1 ${ }^{\circ} \mathrm{C} .{ }^{1} \mathrm{H}$ NMR $\left(400 \mathrm{MHz}, \mathrm{CDCl}_{3}\right) \delta 9.86(\mathrm{~d}, J=6.8 \mathrm{~Hz}, 1 \mathrm{H}), 8.10(\mathrm{~d}, J=9.0 \mathrm{~Hz}, 1 \mathrm{H})$, $8.10(\mathrm{~d}, J=9.0 \mathrm{~Hz}, 1 \mathrm{H}), 7.95(\mathrm{dd}, J=9.1,4.9 \mathrm{~Hz}, 2 \mathrm{H}), 7.95(\mathrm{dd}, J=9.1,4.9 \mathrm{~Hz}, 2 \mathrm{H}), 7.40(\mathrm{dd}, J=9.0$, $1.8 \mathrm{~Hz}, 1 \mathrm{H}), 7.40(\mathrm{dd}, J=9.0,1.8 \mathrm{~Hz}, 1 \mathrm{H}), 7.25(\mathrm{t}, J=7.6 \mathrm{~Hz}, 1 \mathrm{H}), 7.19(\mathrm{dd}, J=9.8,4.1 \mathrm{~Hz}, 1 \mathrm{H}), 4.48$ 
$(\mathrm{q}, J=7.1 \mathrm{~Hz}, 2 \mathrm{H}), 1.50(\mathrm{t}, J=7.1 \mathrm{~Hz}, 3 \mathrm{H}) ;{ }^{13} \mathrm{C} \mathrm{NMR}\left(101 \mathrm{MHz}, \mathrm{CDCl}_{3}\right) \delta 162.0,131.5,128.8,128.6$, 127.5, 126.1, 121.7, 121.2, 118.8, 118.4, 117.5, 117.4, 104.0, 59.6, 14.7; MS (ESI): $\mathrm{m} / \mathrm{z}=273.04$ $[\mathrm{M}]^{+} ; \mathrm{IR}(\mathrm{KBr}): 2981,1661,1428,1184,1121,1044 \mathrm{~cm}^{-1}$.

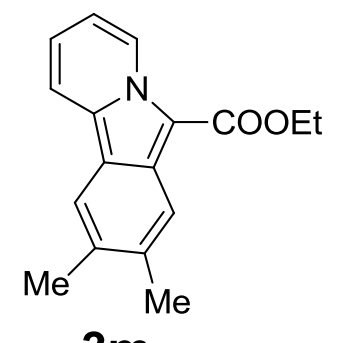

$3 m$

Ethyl 8,9-dimethylpyrido[2,1-a]isoindole-6-carboxylate (3m) : yellow solid; $40.6 \mathrm{mg}, 76 \%$ yield; m.p. 101.1-102.8 ${ }^{\circ} \mathrm{C} .{ }^{1} \mathrm{H}$ NMR $\left(400 \mathrm{MHz}, \mathrm{CDCl}_{3}\right) \delta 9.90(\mathrm{~d}, J=6.4 \mathrm{~Hz}, 1 \mathrm{H}), 8.01(\mathrm{~d}, J=8.4$ $\mathrm{Hz}, 2 \mathrm{H}), 7.80$ (s, 1H), $7.26-7.20(\mathrm{~m}, 1 \mathrm{H}), 7.13(\mathrm{t}, J=6.9 \mathrm{~Hz}, 1 \mathrm{H}), 4.51$ (q, $J=7.1 \mathrm{~Hz}, 2 \mathrm{H}), 2.47$ (s, $3 \mathrm{H}), 2.43(\mathrm{~s}, 3 \mathrm{H}), 1.52(\mathrm{t}, J=7.1 \mathrm{~Hz}, 3 \mathrm{H}) ;{ }^{13} \mathrm{C} \mathrm{NMR}\left(101 \mathrm{MHz}, \mathrm{CDCl}_{3}\right) \delta 162.5,138.2,132.4,130.2$, 127.5, 121.1, 119.4, 118.8, 117.7, 117.1, 116.5, 103.3, 59.3, 21.3, 20.4, 14.9; HR-MS (ESI) calcd for $[\mathrm{M}+1]^{+}: \mathrm{C}_{17} \mathrm{H}_{17} \mathrm{NO}_{2}: 268.1332$, found: 268.1335; IR (KBr): 2979, 1661, 1447, 1325, 1182, 1119 , $1048 \mathrm{~cm}^{-1}$.<smiles>CCOC(=O)c1c2cccc(C)c2c2ccccn12</smiles>

$3 n$

Ethyl 10-methylpyrido[2,1-a]isoindole-6-carboxylate (3n) ${ }^{[5]}$ : yellow solid; $43.5 \mathrm{mg}$, $86 \%$ yield; m.p. 75.7-77.1 ${ }^{\circ} \mathrm{C} .{ }^{1} \mathrm{H}$ NMR $\left(400 \mathrm{MHz}, \mathrm{CDCl}_{3}\right) \delta 10.07(\mathrm{~d}, J=7.0 \mathrm{~Hz}, 1 \mathrm{H}), 8.26(\mathrm{~d}, J=8.7 \mathrm{~Hz}, 1 \mathrm{H})$, $8.19(\mathrm{~d}, J=8.5 \mathrm{~Hz}, 1 \mathrm{H}), 7.43(\mathrm{dd}, J=8.3,7.1 \mathrm{~Hz}, 1 \mathrm{H}), 7.31-7.25(\mathrm{~m}, 1 \mathrm{H}), 7.20(\mathrm{td}, J=6.9,1.2 \mathrm{~Hz}$, $1 \mathrm{H}), 7.03(\mathrm{~d}, J=6.9 \mathrm{~Hz}, 1 \mathrm{H}), 4.51(\mathrm{q}, J=7.1 \mathrm{~Hz}, 2 \mathrm{H}), 2.86(\mathrm{~s}, 3 \mathrm{H}), 1.52(\mathrm{t}, J=7.1 \mathrm{~Hz}, 3 \mathrm{H}) ;{ }^{13} \mathrm{C}$ NMR $\left(101 \mathrm{MHz}, \mathrm{CDCl}_{3}\right) \delta 162.6,133.1,131.9,131.4,127.7,127.4,122.2,121.4,119.9,117.8,117.6,116.5$, 104.2, 59.5, 21.6, 14.8; MS (ESI): m/z = 253.10 [M] ; IR (KBr): 2977, 1661, 1428, 1315, 1213, $1121,1038 \mathrm{~cm}^{-1}$.<smiles>CCOC(=O)c1c2sccc2c2ccccn12</smiles>

30 
Ethyl thieno[3,2-a]indolizine-9-carboxylate (3o) : White solid; $29.9 \mathrm{mg}, 61 \%$ yield; m.p. 86.5$87.4{ }^{\circ} \mathrm{C} .{ }^{1} \mathrm{H}$ NMR $\left(400 \mathrm{MHz}, \mathrm{CDCl}_{3}\right) \delta 9.60(\mathrm{~d}, J=6.3 \mathrm{~Hz}, 1 \mathrm{H}), 7.78(\mathrm{~d}, J=8.7 \mathrm{~Hz}, 1 \mathrm{H}), 7.37(\mathrm{~d}, J=$ $5.3 \mathrm{~Hz}, 1 \mathrm{H}), 7.19-7.12(\mathrm{~m}, 2 \mathrm{H}), 6.97(\mathrm{t}, J=7.0 \mathrm{~Hz}, 1 \mathrm{H}), 4.46(\mathrm{q}, J=7.1 \mathrm{~Hz}, 2 \mathrm{H}), 1.48(\mathrm{t}, J=7.1 \mathrm{~Hz}$, $3 \mathrm{H}) ;{ }^{13} \mathrm{C}$ NMR (101 MHz, $\left.\mathrm{CDCl}_{3}\right) \delta$ 160.6, 129.5, 127.6, 125.0, 123.4, 121.6, 117.3, 116.4, 113.7, 59.9, 14.7; HR-MS (ESI) calcd for $[\mathrm{M}+1]^{+}: \mathrm{C}_{13} \mathrm{H}_{11} \mathrm{NO}_{2} \mathrm{~S}: 246.0583$, found: 246.0587; IR (KBr): 2977 , $1668,1423,1331,1195,1119,1038 \mathrm{~cm}^{-1}$.<smiles>CCOC(=O)c1c2ccsc2c2ccccn12</smiles>

$3 p$

Ethyl thieno[2,3-a]indolizine-4-carboxylate (3p) ${ }^{[5]}$ : White solid; $25.9 \mathrm{mg}, 53 \%$ yield; m.p. 78.2-79.4 ${ }^{\circ} \mathrm{C} .{ }^{1} \mathrm{H}$ NMR $\left(400 \mathrm{MHz}, \mathrm{CDCl}_{3}\right) \delta 9.74(\mathrm{~d}, J=6.9 \mathrm{~Hz}, 1 \mathrm{H}), 7.70(\mathrm{~d}, J=8.8 \mathrm{~Hz}, 1 \mathrm{H}), 7.52(\mathrm{~d}$, $J=5.1 \mathrm{~Hz}, 1 \mathrm{H}), 7.48(\mathrm{~d}, J=5.0 \mathrm{~Hz}, 1 \mathrm{H}), 7.16(\mathrm{t}, J=7.8 \mathrm{~Hz}, 1 \mathrm{H}), 6.99(\mathrm{t}, J=6.8 \mathrm{~Hz}, 1 \mathrm{H}), 4.47(\mathrm{q}, J=$ $7.2 \mathrm{~Hz}, 2 \mathrm{H}), 1.49(\mathrm{t}, J=7.1 \mathrm{~Hz}, 3 \mathrm{H}) ;{ }^{13} \mathrm{C} \mathrm{NMR}\left(101 \mathrm{MHz}, \mathrm{CDCl}_{3}\right) \delta 161.2,140.8,131.3,129.4,128.4$, 121.3, 118.3, 117.1, 116.3, 113.8, 104.2, 59.6, 14.7; MS (ESI): $\mathrm{m} / \mathrm{z}=245.04[\mathrm{M}]^{+} ; \mathrm{IR}(\mathrm{KBr}):=2978$, $1670,1415,1338,1217,1123,1093 \mathrm{~cm}^{-1}$.<smiles>CCOC(=O)c1c2ccccc2c2ccc(C)cn12</smiles>

$3 q$

Ethyl 3-methylpyrido[2,1-a]isoindole-6-carboxylate (3q) : yellow solid; $40.5 \mathrm{mg}$, $80 \%$ yield; m.p. 72.6-73.9 ${ }^{\circ} \mathrm{C} .{ }^{1} \mathrm{H}$ NMR $\left(400 \mathrm{MHz}, \mathrm{CDCl}_{3}\right) \delta 9.82(\mathrm{~s}, 1 \mathrm{H}), 8.24(\mathrm{~d}, J=8.5 \mathrm{~Hz}, 1 \mathrm{H}), 8.04(\mathrm{~d}, J=$ $8.2 \mathrm{~Hz}, 1 \mathrm{H}), 7.99(\mathrm{~d}, J=8.7 \mathrm{~Hz}, 1 \mathrm{H}), 7.56-7.49(\mathrm{~m}, 1 \mathrm{H}), 7.28-7.23(\mathrm{~m}, 1 \mathrm{H}), 7.13(\mathrm{~d}, J=8.7 \mathrm{~Hz}$, $1 \mathrm{H}), 4.50(\mathrm{q}, J=7.1 \mathrm{~Hz}, 2 \mathrm{H}), 2.46(\mathrm{~s}, 3 \mathrm{H}), 1.52(\mathrm{t}, J=7.1 \mathrm{~Hz}, 3 \mathrm{H}) ;{ }^{13} \mathrm{C}$ NMR $\left(101 \mathrm{MHz}, \mathrm{CDCl}_{3}\right) \delta$ 162.6, 131.5, 130.9, 127.7, 127.4, 126.0, 124.4, 120.5, 119.8, 119.2, 118.7, 116.7, 103.5, 59.4, 19.0, 14.8; HR-MS (ESI) calcd for $[\mathrm{M}+1]^{+}: \mathrm{C}_{16} \mathrm{H}_{15} \mathrm{NO}_{2}$ : 254.1176, found: 254.1178; IR (KBr): 2975, $1665,1441,1329,1172,1123,1051 \mathrm{~cm}^{-1}$. 
<smiles>CCOC(=O)c1c2ccccc2c2ccc(OC)cn12</smiles>

Ethyl 3-methoxypyrido[2,1-a]isoindole-6-carboxylate (3r) ${ }^{[5]}$ : yellow solid; $39.3 \mathrm{mg}$, $73 \%$ yield; m.p. 99.9-101.2 ${ }^{\circ} \mathrm{C} .{ }^{1} \mathrm{H}$ NMR $\left(400 \mathrm{MHz}, \mathrm{CDCl}_{3}\right) \delta 9.72(\mathrm{~s}, 1 \mathrm{H}), 8.23(\mathrm{~d}, J=8.4 \mathrm{~Hz}, 1 \mathrm{H}), 8.00(\mathrm{dd}, J=$ 12.2, $8.8 \mathrm{~Hz}, 2 \mathrm{H}), 7.53-7.47(\mathrm{~m}, 1 \mathrm{H}), 7.27-7.22(\mathrm{~m}, 1 \mathrm{H}), 7.06(\mathrm{dd}, J=9.3,2.2 \mathrm{~Hz}, 1 \mathrm{H}), 4.51(\mathrm{q}, J=$ $7.1 \mathrm{~Hz}, 2 \mathrm{H}), 3.97$ (s, 3H), $1.53(\mathrm{t}, J=7.1 \mathrm{~Hz}, 3 \mathrm{H}) ;{ }^{13} \mathrm{C} \mathrm{NMR}\left(101 \mathrm{MHz}, \mathrm{CDCl}_{3}\right) \delta 162.7,152.8,130.7$, $128.8,127.1,120.8,119.7,119.0,118.7,117.3,115.4,110.7,104.6,59.4,56.0,14.8 ; \mathrm{MS}$ (ESI): $\mathrm{m} / \mathrm{z}=$ 269.04 [M] $]^{+}$IR (KBr): 2981, 1657, 1429, 1317, 1168, 1128, $1041 \mathrm{~cm}^{-1}$.<smiles>CCOC(=O)c1c2ccccc2c2ccc(Cl)cn12</smiles>

3s

Ethyl 3-chloropyrido[2,1-a]isoindole-6-carboxylate (3s) : yellow solid; $48.6 \mathrm{mg}$, $89 \%$ yield; m.p. 94.2-95.7 ${ }^{\circ} \mathrm{C} .{ }^{1} \mathrm{H}$ NMR (400 MHz, $\left.\mathrm{CDCl}_{3}\right) \delta 10.07(\mathrm{~s}, 1 \mathrm{H}), 8.26(\mathrm{~d}, J=8.5 \mathrm{~Hz}, 1 \mathrm{H}), 8.06(\mathrm{dd}, J=$ 8.4, $6.6 \mathrm{~Hz}, 2 \mathrm{H}), 7.58-7.53(\mathrm{~m}, 1 \mathrm{H}), 7.30(\mathrm{t}, J=7.5 \mathrm{~Hz}, 1 \mathrm{H}), 7.26(\mathrm{dd}, J=6.2,2.9 \mathrm{~Hz}, 1 \mathrm{H}), 4.52(\mathrm{q}, J$ $=7.1 \mathrm{~Hz}, 2 \mathrm{H}), 1.53(\mathrm{t}, J=7.1 \mathrm{~Hz}, 3 \mathrm{H}) .{ }^{13} \mathrm{C} \mathrm{NMR}\left(101 \mathrm{MHz}, \mathrm{CDCl}_{3}\right) \delta 162.3,130.7,130.5,128.1$, 125.5, 125.3 122.0, 121.4, 119.9, 119.1, 118.7, 117.4, 104.7, 59.8, 14.7; HR-MS (ESI) calcd for [M + $1^{+}: \mathrm{C}_{15} \mathrm{H}_{12} \mathrm{ClNO}_{2}$ : 274.0629, found: 274.0633; IR (KBr): 2979, 1658, 1439, 1321, 1177, 1109 , $1038 \mathrm{~cm}^{-1}$.<smiles>CCOC(=O)c1c2ccccc2c2ccc(C(F)(F)F)cn12</smiles>

$3 t$

Ethyl 3-(trifluoromethyl)pyrido[2,1-a]isoindole-6-carboxylate (3t) : yellow solid; $56.5 \mathrm{mg}, 92 \%$ yield; m.p. $131.0-132.3{ }^{\circ} \mathrm{C} .{ }^{1} \mathrm{H}$ NMR (400 MHz, $\left.\mathrm{CDCl}_{3}\right) \delta 10.34$ (s, $\left.1 \mathrm{H}\right), 8.25(\mathrm{~d}, J=8.6 \mathrm{~Hz}, 1 \mathrm{H})$, $8.15(\mathrm{~d}, J=9.0 \mathrm{~Hz}, 1 \mathrm{H}), 8.07(\mathrm{~d}, J=8.3 \mathrm{~Hz}, 1 \mathrm{H}), 7.59-7.54(\mathrm{~m}, 1 \mathrm{H}), 7.38(\mathrm{dd}, J=9.0,1.2 \mathrm{~Hz}, 1 \mathrm{H})$, 
$7.33-7.28(\mathrm{~m}, 1 \mathrm{H}), 4.52(\mathrm{q}, J=7.1 \mathrm{~Hz}, 2 \mathrm{H}), 1.53(\mathrm{t}, J=7.1 \mathrm{~Hz}, 3 \mathrm{H}) ;{ }^{13} \mathrm{C} \mathrm{NMR}\left(101 \mathrm{MHz}, \mathrm{CDCl}_{3}\right) \delta$ 162.2, 131.8 (d, J = 77.8 Hz), 128.8, 125.7, 123.6 (d, J = 272.9 Hz), 121.8, 121.0, 120.7, 120.1, 119.5, 118.4, 117.8, 116.5, 105.6, 60.0, 14.6; HR-MS (ESI) calcd for $[\mathrm{M}+1]^{+}: \mathrm{C}_{16} \mathrm{H}_{12} \mathrm{~F}_{3} \mathrm{NO}_{2}$ : 308.0893, found: 308.0896; IR (KBr): 2975, 1657, 1432, 1321, 1179, 1125, $1057 \mathrm{~cm}^{-1}$.

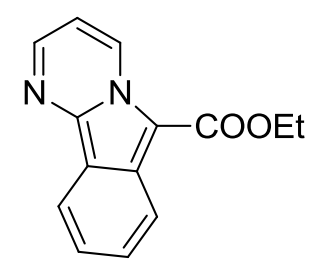

\section{$3 u$}

Ethyl pyrimido[2,1-a]isoindole-6-carboxylate (3u) : yellow solid; $31.2 \mathrm{mg}, 61 \%$ yield; m.p. 87.1-88.2 ${ }^{\circ} \mathrm{C} .{ }^{1} \mathrm{H}$ NMR $\left(400 \mathrm{MHz}, \mathrm{CDCl}_{3}\right) \delta 10.13(\mathrm{~d}, J=6.8 \mathrm{~Hz}, 1 \mathrm{H}), 8.51(\mathrm{dd}, J=4.1,1.6 \mathrm{~Hz}, 1 \mathrm{H})$, $8.40(\mathrm{~d}, J=8.3 \mathrm{~Hz}, 1 \mathrm{H}), 8.27(\mathrm{~d}, J=8.5 \mathrm{~Hz}, 1 \mathrm{H}), 7.67-7.60(\mathrm{~m}, 1 \mathrm{H}), 7.40-7.33(\mathrm{~m}, 1 \mathrm{H}), 7.23(\mathrm{dd}, J$ $=7.1,4.2 \mathrm{~Hz}, 1 \mathrm{H}), 4.52(\mathrm{q}, J=7.1 \mathrm{~Hz}, 2 \mathrm{H}), 1.53(\mathrm{t}, J=7.1 \mathrm{~Hz}, 3 \mathrm{H}) ;{ }^{13} \mathrm{C} \mathrm{NMR}\left(101 \mathrm{MHz}, \mathrm{CDCl}_{3}\right) \delta$ 162.5, 143.5, 139.3, 133.0, 131.6, 129.6, 121.8, 120.7, 119.6, 117.2, 112.5, 101.3, 59.9, 14.7; HR-MS (ESI) calcd for $[\mathrm{M}+1]^{+}: \mathrm{C}_{14} \mathrm{H}_{12} \mathrm{~N}_{2} \mathrm{O}_{3}: 257.0921$, found: 257.0927; IR (KBr): 2977, 1661, 1435, $1338,1168,1119,1043 \mathrm{~cm}^{-1}$.<smiles>CCOC(=O)c1c2ccccc2c2c(C)cccn12</smiles>

$3 \mathbf{v}$

Ethyl 1-methylpyrido[2,1-a]isoindole-6-carboxylate (3v) ${ }^{[5]}$ : yellow solid; $42.0 \mathrm{mg}$, 83\% yield; m.p. 83.2-84.5 ${ }^{\circ} \mathrm{C} .{ }^{1} \mathrm{H}$ NMR $\left(400 \mathrm{MHz}, \mathrm{CDCl}_{3}\right) \delta 9.98(\mathrm{~d}, J=6.7 \mathrm{~Hz}, 1 \mathrm{H}), 8.35(\mathrm{~d}, J=8.5 \mathrm{~Hz}, 1 \mathrm{H})$, $8.25(\mathrm{~d}, J=8.4 \mathrm{~Hz}, 1 \mathrm{H}), 7.57-7.50(\mathrm{~m}, 1 \mathrm{H}), 7.29-7.24(\mathrm{~m}, 1 \mathrm{H}), 7.13(\mathrm{td}, J=7.0,2.9 \mathrm{~Hz}, 1 \mathrm{H}), 7.08$ $(\mathrm{d}, J=6.5 \mathrm{~Hz}, 1 \mathrm{H}), 4.51(\mathrm{q}, J=7.1 \mathrm{~Hz}, 2 \mathrm{H}), 2.89(\mathrm{~s}, 3 \mathrm{H}), 1.53(\mathrm{dd}, J=8.6,5.6 \mathrm{~Hz}, 3 \mathrm{H}) ;{ }^{13} \mathrm{C} \mathrm{NMR}$ $\left(101 \mathrm{MHz}, \mathrm{CDCl}_{3}\right) \delta 162.5,131.7,131.2,130.5,127.2,125.5,123.1,122.0,120.6,119.7,119.4,116.6$, 104.0, 59.5, 20.7, 14.8; MS (ESI): m/z = 253.10 [M] ${ }^{+}$; IR (KBr): 2976, 1661, 1447, 1327, 1183, $1114,1053 \mathrm{~cm}^{-1}$.<smiles>CCOC(=O)c1c2ccccc2c2cc(C)ccn12</smiles> 
Ethyl 2-methylpyrido[2,1-a]isoindole-6-carboxylate (3w) : yellow solid; $42.5 \mathrm{mg}$, $84 \%$ yield; m.p. $79.2-80.5{ }^{\circ} \mathrm{C} .{ }^{1} \mathrm{H}$ NMR $\left(400 \mathrm{MHz}, \mathrm{CDCl}_{3}\right) \delta 9.88(\mathrm{~d}, J=6.8 \mathrm{~Hz}, 1 \mathrm{H}), 8.24(\mathrm{~d}, J=8.5 \mathrm{~Hz}, 1 \mathrm{H})$, $8.05(\mathrm{~d}, J=8.2 \mathrm{~Hz}, 1 \mathrm{H}), 7.89(\mathrm{~s}, 1 \mathrm{H}), 7.55-7.48(\mathrm{~m}, 1 \mathrm{H}), 7.26-7.21(\mathrm{~m}, 1 \mathrm{H}), 7.10-7.01(\mathrm{~m}, 1 \mathrm{H})$, $4.50(\mathrm{q}, J=7.1 \mathrm{~Hz}, 2 \mathrm{H}), 2.52(\mathrm{~s}, 3 \mathrm{H}), 1.51(\mathrm{t}, J=7.1 \mathrm{~Hz}, 3 \mathrm{H}) ;{ }^{13} \mathrm{C} \mathrm{NMR}\left(101 \mathrm{MHz}, \mathrm{CDCl}_{3}\right) \delta 162.5$, 133.2, 133.0, 131.4, 128.1, 127.2, 120.2, 119.7, 119.5, 118.0, 116.6, 103.1, 59.3, 21.1, 14.8; HR-MS (ESI) calcd for $[\mathrm{M}+1]^{+}: \mathrm{C}_{16} \mathrm{H}_{15} \mathrm{NO}_{2}: 254.1176$, found: 254.1179; IR (KBr): 2975, 1663, 1439, $1318,1188,1122,1032 \mathrm{~cm}^{-1}$.<smiles>CCOC(=O)c1cc(C)c2ccccn12</smiles>

$3 x$

Ethyl 1-methylindolizine-3-carboxylate (3x) : white oil; $25.6 \mathrm{mg}, 63 \%$ yield. ${ }^{1} \mathrm{H} \mathrm{NMR}(400 \mathrm{MHz}$, $\left.\mathrm{CDCl}_{3}\right) \delta 9.39(\mathrm{~d}, J=7.1 \mathrm{~Hz}, 1 \mathrm{H}), 7.42(\mathrm{~d}, J=8.9 \mathrm{~Hz}, 1 \mathrm{H}), 7.35(\mathrm{~s}, 1 \mathrm{H}), 6.97(\mathrm{t}, J=7.7 \mathrm{~Hz}, 1 \mathrm{H}), 6.76$ $(\mathrm{t}, J=6.8 \mathrm{~Hz}, 1 \mathrm{H}), 4.38(\mathrm{q}, J=7.1 \mathrm{~Hz}, 2 \mathrm{H}), 2.35(\mathrm{~s}, 3 \mathrm{H}), 1.42(\mathrm{t}, J=7.1 \mathrm{~Hz}, 3 \mathrm{H}) ;{ }^{13} \mathrm{C}$ NMR $(101 \mathrm{MHz}$, $\left.\mathrm{CDCl}_{3}\right) \delta 161.3,136.1,127.2,121.6,120.3,116.9,112.5,112.3,110.0,59.5,14.6,10.3$; HR-MS (ESI) calcd for $[\mathrm{M}+1]^{+}: \mathrm{C}_{12} \mathrm{H}_{13} \mathrm{NO}_{2}: 204.0941$, found: 204.0944; IR (KBr): 2978, 1682, 1469, 1377, $1301,1214,1077 \mathrm{~cm}^{-1}$.

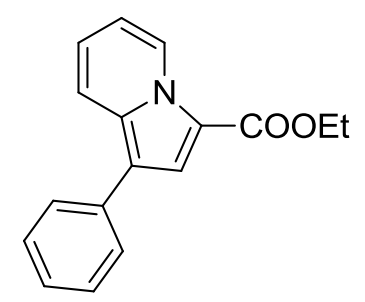

\section{3y}

Ethyl 1-phenylindolizine-3-carboxylate (3y) : white oil; $36.6 \mathrm{mg}, 69 \%$ yield. ${ }^{1} \mathrm{H} \mathrm{NMR}(400 \mathrm{MHz}$, $\left.\mathrm{CDCl}_{3}\right) \delta 9.48(\mathrm{~d}, J=7.1 \mathrm{~Hz}, 1 \mathrm{H}), 7.81(\mathrm{~d}, J=9.0 \mathrm{~Hz}, 1 \mathrm{H}), 7.67(\mathrm{~s}, 1 \mathrm{H}), 7.58(\mathrm{~d}, J=7.4 \mathrm{~Hz}, 2 \mathrm{H}), 7.44$ $(\mathrm{t}, J=7.5 \mathrm{~Hz}, 2 \mathrm{H}), 7.29(\mathrm{t}, J=7.4 \mathrm{~Hz}, 1 \mathrm{H}), 7.06(\mathrm{t}, J=7.7 \mathrm{~Hz}, 1 \mathrm{H}), 6.83(\mathrm{t}, J=6.8 \mathrm{~Hz}, 1 \mathrm{H}), 4.40(\mathrm{q}, J$ $=7.1 \mathrm{~Hz}, 2 \mathrm{H}), 1.42(\mathrm{t}, J=7.1 \mathrm{~Hz}, 3 \mathrm{H}) ;{ }^{13} \mathrm{C} \mathrm{NMR}\left(101 \mathrm{MHz}, \mathrm{CDCl}_{3}\right) \delta 161.4,135.1,134.7,128.8$, $127.8,127.5,126.2,122.3,120.6,117.8,116.3,113.9,113.0,59.8,14.6$; HR-MS (ESI) calcd for [M + $1]^{+}: \mathrm{C}_{17} \mathrm{H}_{15} \mathrm{NO}_{2}$ : 266.1124, found: 266.1127; IR (KBr): 2979, 1678, 1454, 1357, 1289, 1207 , $1053 \mathrm{~cm}^{-1}$. 


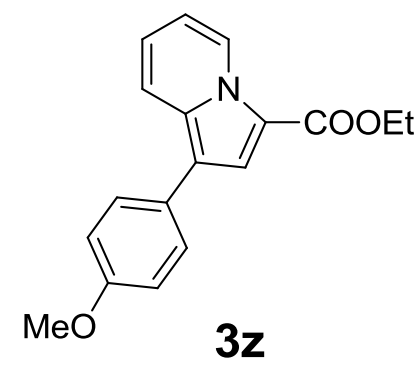

Ethyl 1-(4-methoxyphenyl)indolizine-3-carboxylate (3z) : white solid; $43.7 \mathrm{mg}, 74 \%$ yield; m.p. 87.4-88.6 ${ }^{\circ} \mathrm{C} .{ }^{1} \mathrm{H}$ NMR $\left(400 \mathrm{MHz}, \mathrm{CDCl}_{3}\right) \delta 9.46(\mathrm{~d}, J=7.1 \mathrm{~Hz}, 1 \mathrm{H}), 7.75(\mathrm{~d}, J=9.0 \mathrm{~Hz}, 1 \mathrm{H}), 7.60(\mathrm{~s}$, $1 \mathrm{H}), 7.49(\mathrm{~d}, J=8.3 \mathrm{~Hz}, 2 \mathrm{H}), 7.01(\mathrm{dd}, J=18.3,7.9 \mathrm{~Hz}, 3 \mathrm{H}), 6.81(\mathrm{t}, J=6.9 \mathrm{~Hz}, 1 \mathrm{H}), 4.39(\mathrm{q}, J=7.1$ $\mathrm{Hz}, 2 \mathrm{H}), 3.85(\mathrm{~s}, 3 \mathrm{H}), 1.41(\mathrm{t}, J=7.1 \mathrm{~Hz}, 3 \mathrm{H}) ;{ }^{13} \mathrm{C} \mathrm{NMR}\left(101 \mathrm{MHz}, \mathrm{CDCl}_{3}\right) \delta 161.4,158.2,134.6$, 129.0, 127.6, 127.5, 121.9, 120.4, 117.7, 116.1, 114.3, 113.7, 112.9, 59.8, 55.3, 14.6; HR-MS (ESI) calcd for $[\mathrm{M}+1]^{+}: \mathrm{C}_{18} \mathrm{H}_{17} \mathrm{NO}_{3}: 296.1281$, found: 296.1280; IR (KBr): 2977, 1684, 1469, 1374 , $1301,1207,1055 \mathrm{~cm}^{-1}$.<smiles>CCOC(=O)c1cc(-c2ccc(Cl)cc2)c2ccccn12</smiles>

Ethyl 1-(4-chlorophenyl)indolizine-3-carboxylate (3az) : white solid; $34.7 \mathrm{mg}$, 58\% yield; m.p. 107.1-108.9 ${ }^{\circ} \mathrm{C} .{ }^{1} \mathrm{H}$ NMR (400 MHz, $\left.\mathrm{CDCl}_{3}\right) \delta 9.48(\mathrm{~d}, J=7.1 \mathrm{~Hz}, 1 \mathrm{H}), 7.75$ (d, J=9.0 Hz, 1H), 7.64 (s, 1H), $7.50(\mathrm{~d}, J=8.4 \mathrm{~Hz}, 2 \mathrm{H}), 7.41(\mathrm{~d}, J=8.4 \mathrm{~Hz}, 2 \mathrm{H}), 7.12-7.04(\mathrm{~m}, 1 \mathrm{H}), 6.85(\mathrm{t}, J=6.7 \mathrm{~Hz}, 1 \mathrm{H})$, $4.40(\mathrm{q}, J=7.1 \mathrm{~Hz}, 2 \mathrm{H}), 1.42(\mathrm{t}, J=7.1 \mathrm{~Hz}, 3 \mathrm{H}) ;{ }^{13} \mathrm{C} \mathrm{NMR}\left(101 \mathrm{MHz}, \mathrm{CDCl}_{3}\right) \delta$ 161.3, 134.6, 133.6, 131.9, 129.0, 128.9, 127.6, 122.6, 120.5, 117.5, 114.9, 114.1, 113.1, 59.9, 14.5; HR-MS (ESI) calcd for $[\mathrm{M}+1]^{+}: \mathrm{C}_{17} \mathrm{H}_{14} \mathrm{ClNO}_{2}: 300.0786$, found: 300.0790; IR (KBr): 2979, 1677, 1439, 1372, 1306 , $1222,1063 \mathrm{~cm}^{-1}$.<smiles>CCOC(=O)c1c2c(c3ccccn13)CCC2</smiles>

$3 \mathrm{bz}$

Ethyl 2,3-dihydro-1H-cyclopenta[a]indolizine-4-carboxylate (3bz) : white oil; $27.5 \mathrm{mg}, 60 \%$ yield. ${ }^{1} \mathrm{H}$ NMR $\left(400 \mathrm{MHz}, \mathrm{CDCl}_{3}\right) \delta 9.35(\mathrm{~d}, J=7.1 \mathrm{~Hz}, 1 \mathrm{H}), 7.27(\mathrm{~d}, J=8.2 \mathrm{~Hz}, 1 \mathrm{H}), 6.96-6.89(\mathrm{~m}$, $1 \mathrm{H}), 6.65(\mathrm{t}, J=6.9 \mathrm{~Hz}, 1 \mathrm{H}), 4.34(\mathrm{q}, J=7.1 \mathrm{~Hz}, 2 \mathrm{H}), 3.07(\mathrm{t}, J=7.2 \mathrm{~Hz}, 2 \mathrm{H}), 2.86(\mathrm{t}, J=7.1 \mathrm{~Hz}, 2 \mathrm{H})$, $2.50-2.42(\mathrm{~m}, 2 \mathrm{H}), 1.38(\mathrm{t}, J=7.1 \mathrm{~Hz}, 3 \mathrm{H}) ;{ }^{13} \mathrm{C} \mathrm{NMR}\left(101 \mathrm{MHz}, \mathrm{CDCl}_{3}\right) \delta 161.7,144.6,131.1,128.1$, 
121.2, 121.0, 116.8, 111.1, 107.1, 59.3, 29.9, 28.3, 24.9, 14.6; HR-MS (ESI) calcd for $[\mathrm{M}+1]^{+}$: $\mathrm{C}_{14} \mathrm{H}_{15} \mathrm{NO}_{2}$ : 230.1176, found: 230.1176; IR (KBr): 2973, 1680, 1443, 1359, 1221, 1089, $1037 \mathrm{~cm}^{-}$ 1.

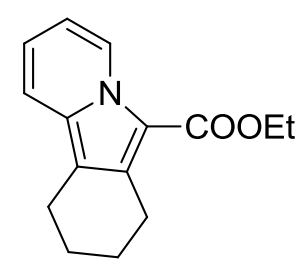

$3 c z$

Ethyl 7,8,9,10-tetrahydropyrido[2,1-a]isoindole-6-carboxylate (3cz) : white oil; $32.1 \mathrm{mg}, 66 \%$ yield. ${ }^{1} \mathrm{H}$ NMR $\left(400 \mathrm{MHz}, \mathrm{CDCl}_{3}\right) \delta 9.43(\mathrm{~d}, J=7.1 \mathrm{~Hz}, 1 \mathrm{H}), 7.30(\mathrm{~d}, J=8.7 \mathrm{~Hz}, 1 \mathrm{H}), 6.94(\mathrm{t}, J=7.6$ $\mathrm{Hz}, 1 \mathrm{H}), 6.69(\mathrm{t}, J=6.8 \mathrm{~Hz}, 1 \mathrm{H}), 4.36(\mathrm{q}, J=6.7 \mathrm{~Hz}, 2 \mathrm{H}), 3.01(\mathrm{~s}, 2 \mathrm{H}), 2.70(\mathrm{~s}, 2 \mathrm{H}), 1.85(\mathrm{~s}, 4 \mathrm{H}), 1.39$ $(\mathrm{t}, J=7.0 \mathrm{~Hz}, 3 \mathrm{H}) ;{ }^{13} \mathrm{C} \mathrm{NMR}\left(101 \mathrm{MHz}, \mathrm{CDCl}_{3}\right) \delta 162.2,134.6,134.0,127.4,120.7,115.8,111.8$, 111.7, 109.9, 59.2, 24.5, 23.5, 22.9, 20.7, 14.6; HR-MS (ESI) calcd for $[\mathrm{M}+1]^{+}: \mathrm{C}_{15} \mathrm{H}_{17} \mathrm{NO}_{2}$ : 244.1332, found: 244.1333; IR (KBr): 2973, 1679, 1450, 1369, 1218, 1109, $1041 \mathrm{~cm}^{-1}$.<smiles>CCOC(=O)c1c2c(c3c(C)cccn13)CCCC2</smiles>

$3 d z$

Ethyl 1-methyl-7,8,9,10-tetrahydropyrido[2,1-a]isoindole-6-carboxylate (3dz) : white oil; 29.8 mg, $58 \%$ yield. ${ }^{1} \mathrm{H}$ NMR $\left(400 \mathrm{MHz}, \mathrm{CDCl}_{3}\right) \delta 9.35(\mathrm{~d}, J=7.0 \mathrm{~Hz}, 1 \mathrm{H}), 6.60(\mathrm{~d}, J=6.6 \mathrm{~Hz}, 1 \mathrm{H}), 6.52$ (t, $J=6.9 \mathrm{~Hz}, 1 \mathrm{H}), 4.35(\mathrm{q}, J=7.1 \mathrm{~Hz}, 2 \mathrm{H}), 3.04$ (s, 2H), 2.99 (s, 2H), 2.56 (s, 3H), 1.79 (s, 4H), 1.39 $(\mathrm{t}, J=7.1 \mathrm{~Hz}, 3 \mathrm{H}) ;{ }^{13} \mathrm{C} \mathrm{NMR}\left(101 \mathrm{MHz}, \mathrm{CDCl}_{3}\right) \delta 162.2,134.7,133.9,128.0,125.4,121.4,112.5$, 111.4, 110.0, 59.2, 24.8, 23.9, 23.6, 22.9, 20.0, 14.6; HR-MS (ESI) calcd for $[\mathrm{M}+1]^{+}: \mathrm{C}_{16} \mathrm{H}_{19} \mathrm{NO}_{2}$ : 258.1489, found: 258.1487; IR (KBr): 2975, 1680, 1453, 1358, 1221, 1100, $1032 \mathrm{~cm}^{-1}$.<smiles>CCOC(=O)c1c2c(c3cc(C)ccn13)CCCC2</smiles>

3ez

Ethyl 2-methyl-7,8,9,10-tetrahydropyrido[2,1-a]isoindole-6-carboxylate (3ez) : white oil; 32.9 $\mathrm{mg}, 64 \%$ yield. ${ }^{1} \mathrm{H}$ NMR $\left(400 \mathrm{MHz}, \mathrm{CDCl}_{3}\right) \delta 9.32(\mathrm{~d}, J=7.2 \mathrm{~Hz}, 1 \mathrm{H}), 7.06(\mathrm{~s}, 1 \mathrm{H}), 6.54(\mathrm{~d}, J=7.2$ $\mathrm{Hz}, 1 \mathrm{H}), 4.35$ (q, $J=7.1 \mathrm{~Hz}, 2 \mathrm{H}), 2.99$ (s, 2H), 2.67 (s, 2H), 2.34 (s, 3H), 1.84 (s, 4H), 1.39 (t, $J=7.1$ 
$\mathrm{Hz}, 3 \mathrm{H}) ;{ }^{13} \mathrm{C} \mathrm{NMR}\left(101 \mathrm{MHz}, \mathrm{CDCl}_{3}\right) \delta 162.2,135.1,134.3,131.5,126.9,114.4,110.4,109.2,59.1$, 24.5, 23.5, 23.0, 21.1, 20.7, 14.7; HR-MS (ESI) calcd for $[\mathrm{M}+1]^{+}: \mathrm{C}_{16} \mathrm{H}_{19} \mathrm{NO}_{2}: 258.1489$, found: 258.1483; IR (KBr): 2976, 1682, 1459, 1366, 1209, 1098, $1029 \mathrm{~cm}^{-1}$.

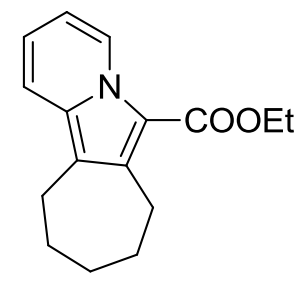

$3 f z$

Ethyl 8,9,10,11-tetrahydro-7H-cyclohepta[a]indolizine-6-carboxylate (3fz) : white oil; 36.5 $\mathrm{mg}, 71 \%$ yield. ${ }^{1} \mathrm{H}$ NMR (400 MHz, $\left.\mathrm{CDCl}_{3}\right) \delta 9.38(\mathrm{~d}, J=7.2 \mathrm{~Hz}, 1 \mathrm{H}), 7.34(\mathrm{~d}, J=8.9 \mathrm{~Hz}, 1 \mathrm{H}), 6.90$ $(\mathrm{t}, J=7.7 \mathrm{~Hz}, 1 \mathrm{H}), 6.62(\mathrm{t}, J=6.8 \mathrm{~Hz}, 1 \mathrm{H}), 4.39(\mathrm{q}, J=7.0 \mathrm{~Hz}, 2 \mathrm{H}), 3.30-3.23(\mathrm{~m}, 2 \mathrm{H}), 2.83-2.76$ $(\mathrm{m}, 2 \mathrm{H}), 1.89(\mathrm{~d}, J=5.1 \mathrm{~Hz}, 2 \mathrm{H}), 1.74-1.64(\mathrm{~m}, 4 \mathrm{H}), 1.41(\mathrm{t}, J=7.1 \mathrm{~Hz}, 3 \mathrm{H}) ;{ }^{13} \mathrm{C}$ NMR $(101 \mathrm{MHz}$, $\left.\mathrm{CDCl}_{3}\right) \delta 162.6,139.0,134.5,127.7,120.7,117.2,115.8,111.2,110.5,59.5,32.7,28.6,28.1,27.3,24.9$, 14.5; HR-MS (ESI) calcd for $[\mathrm{M}+1]^{+}: \mathrm{C}_{16} \mathrm{H}_{1} \mathrm{NO}_{2}: 258.1489$, found: 258.1487; IR (KBr): 2977, $1681,1469,1372,1223,1109,1040 \mathrm{~cm}^{-1}$.

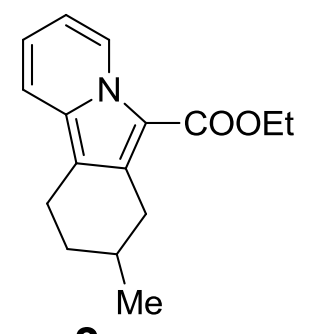

$3 g z$

Ethyl 8-methyl-7,8,9,10-tetrahydropyrido[2,1-a]isoindole-6-carboxylate (3gz) : white oil; 33.9 mg, 66\% yield. ${ }^{1} \mathrm{H}$ NMR $\left(400 \mathrm{MHz}, \mathrm{CDCl}_{3}\right) \delta 9.42(\mathrm{~d}, J=7.2 \mathrm{~Hz}, 1 \mathrm{H}), 7.30(\mathrm{~d}, J=8.8 \mathrm{~Hz}, 1 \mathrm{H}), 6.94$ $(\mathrm{dd}, J=8.2,7.2 \mathrm{~Hz}, 1 \mathrm{H}), 6.73-6.66(\mathrm{~m}, 1 \mathrm{H}), 4.41-4.33(\mathrm{~m}, 2 \mathrm{H}), 3.24(\mathrm{dd}, J=17.7,5.1 \mathrm{~Hz}, 1 \mathrm{H})$, 2.82 (ddd, $J=15.5,5.1,3.2 \mathrm{~Hz}, 1 \mathrm{H}), 2.71-2.59$ (m, 1H), 2.46 (dd, $J=17.7,10.0 \mathrm{~Hz}, 1 \mathrm{H}), 1.93$ (ddd, $J=15.9,8.8,4.5 \mathrm{~Hz}, 2 \mathrm{H}), 1.46(\mathrm{dd}, J=10.9,5.5 \mathrm{~Hz}, 1 \mathrm{H}), 1.40(\mathrm{dd}, J=9.2,5.0 \mathrm{~Hz}, 3 \mathrm{H}), 1.13(\mathrm{~d}, J=$ $6.6 \mathrm{~Hz}, 3 \mathrm{H}) ;{ }^{13} \mathrm{C} \mathrm{NMR}\left(101 \mathrm{MHz}, \mathrm{CDCl}_{3}\right) \delta 162.2,134.4,134.1,127.5,120.7,115.8,111.8,111.4$, 109.8, 59.2, 32.9, 31.2, 29.6, 21.9, 20.3, 14.7; HR-MS (ESI) calcd for $[\mathrm{M}+1]^{+}: \mathrm{C}_{16} \mathrm{H}_{9}{ }_{9} \mathrm{NO}_{2}$ : 258.1476, found: 258.1479; IR (KBr): 2978, 1678, 1469, 1355, 1209, 1092, $1029 \mathrm{~cm}^{-1}$. 


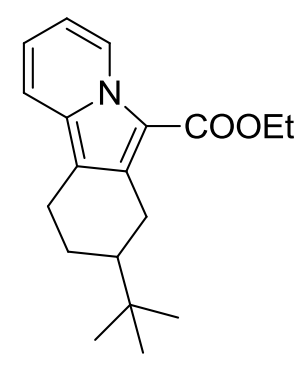

3hz

Ethyl 8-(tert-butyl)-7,8,9,10-tetrahydropyrido[2,1-a]isoindole-6-carboxylate (3hz) : white oil; $43.7 \mathrm{mg}, 73 \%$ yield. ${ }^{1} \mathrm{H}$ NMR (400 MHz, $\left.\mathrm{CDCl}_{3}\right) \delta 9.42(\mathrm{~d}, J=7.2 \mathrm{~Hz}, 1 \mathrm{H}), 7.29(\mathrm{~d}, J=8.8 \mathrm{~Hz}, 1 \mathrm{H})$, $6.97-6.89(\mathrm{~m}, 1 \mathrm{H}), 6.69(\mathrm{td}, J=7.0,1.3 \mathrm{~Hz}, 1 \mathrm{H}), 4.42-4.30(\mathrm{~m}, 2 \mathrm{H}), 3.27(\mathrm{dd}, J=17.9,4.5 \mathrm{~Hz}, 1 \mathrm{H})$, $2.92-2.83(\mathrm{~m}, 1 \mathrm{H}), 2.62-2.51(\mathrm{~m}, 2 \mathrm{H}), 2.10(\mathrm{ddd}, J=12.4,4.9,1.9 \mathrm{~Hz}, 1 \mathrm{H}), 1.49$ (tdd, $J=12.0,4.9$, $1.8 \mathrm{~Hz}, 1 \mathrm{H}), 1.41(\mathrm{t}, J=7.1 \mathrm{~Hz}, 3 \mathrm{H}), 1.33(\mathrm{dd}, J=12.3,5.0 \mathrm{~Hz}, 1 \mathrm{H}), 1.00(\mathrm{~s}, 9 \mathrm{H}) ;{ }^{13} \mathrm{C}$ NMR $(101 \mathrm{MHz}$, $\left.\mathrm{CDCl}_{3}\right) \delta 162.2,135.0,134.3,127.4,120.7,115.8,111.9,111.7,110.0,59.2,45.4,32.6,27.4,26.1,24.5$, 21.7, 14.6; HR-MS (ESI) calcd for [M + 1] $]^{+}: \mathrm{C}_{19} \mathrm{H}_{25} \mathrm{NO}_{2}: 300.1936$, found : 300.1937; IR (KBr): $2973,1682,1482,1368,1223,1108,1051 \mathrm{~cm}^{-1}$.

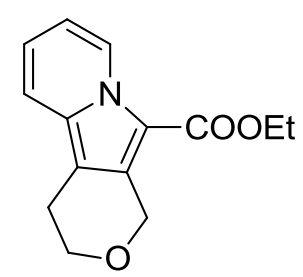

$3 i z$

Ethyl 3,4-dihydro-1H-pyrano[4,3-a]indolizine-10-carboxylate (3iz) : white solid; $22.5 \mathrm{mg}, 46 \%$ yield; m.p. 66.7-67.8 ${ }^{\circ} \mathrm{C} .{ }^{1} \mathrm{H}$ NMR $\left(400 \mathrm{MHz}, \mathrm{CDCl}_{3}\right) \delta 9.42(\mathrm{~d}, J=7.1 \mathrm{~Hz}, 1 \mathrm{H}), 7.36(\mathrm{dd}, J=7.8,1.0$ $\mathrm{Hz}, 1 \mathrm{H}), 7.01$ (ddd, $J=8.8,6.7,1.0 \mathrm{~Hz}, 1 \mathrm{H}), 6.77(\mathrm{td}, J=7.0,1.3 \mathrm{~Hz}, 1 \mathrm{H}), 5.07$ (s, 2H), 4.36 (q, $J=$ $7.1 \mathrm{~Hz}, 2 \mathrm{H}), 4.01(\mathrm{t}, J=5.6 \mathrm{~Hz}, 2 \mathrm{H}), 2.84(\mathrm{t}, J=5.5 \mathrm{~Hz}, 2 \mathrm{H}), 1.40(\mathrm{t}, J=7.1 \mathrm{~Hz}, 3 \mathrm{H}) ;{ }^{13} \mathrm{C}$ NMR $(101$ $\left.\mathrm{MHz}, \mathrm{CDCl}_{3}\right) \delta 161.4,134.5,131.5,127.3,121.3,115.9,112.4,108.0,107.9,65.7,64.8,59.6,21.3$, 14.6; HR-MS (ESI) calcd for $[\mathrm{M}+1]^{+}: \mathrm{C}_{14} \mathrm{H}_{15} \mathrm{NO}_{3}: 246.1172$, found : 246.1176; IR (KBr): 2976, $1679,1465,1378,1236,1112,1045 \mathrm{~cm}^{-1}$.

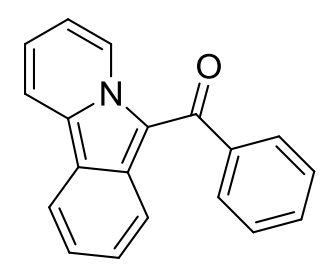

$3 \mathbf{j z}$

Phenyl(pyrido[2,1-a]isoindol-6-yl)methanone (3jz) ${ }^{[6]}$ : yellow solid; $10.3 \mathrm{mg}, 19 \%$ yield; m.p. 101.6-102.8 ${ }^{\circ} \mathrm{C} .{ }^{1} \mathrm{H}$ NMR $\left(400 \mathrm{MHz}, \mathrm{CDCl}_{3}\right) \delta 10.66(\mathrm{~d}, J=7.0 \mathrm{~Hz}, 1 \mathrm{H}), 8.23(\mathrm{~d}, J=8.5 \mathrm{~Hz}, 1 \mathrm{H})$, $8.12(\mathrm{dd}, J=6.6,1.4 \mathrm{~Hz}, 1 \mathrm{H}), 7.71(\mathrm{dd}, J=7.9,1.4 \mathrm{~Hz}, 2 \mathrm{H}), 7.56(\mathrm{dtd}, J=8.3,7.0,5.0 \mathrm{~Hz}, 4 \mathrm{H}), 7.39$ 
$(\mathrm{td}, J=7.0,1.3 \mathrm{~Hz}, 1 \mathrm{H}), 7.29-7.22(\mathrm{~m}, 2 \mathrm{H}), 6.86(\mathrm{dd}, J=7.3,1.3 \mathrm{~Hz}, 1 \mathrm{H}) ;{ }^{13} \mathrm{C}$ NMR $(101 \mathrm{MHz}$, $\left.\mathrm{CDCl}_{3}\right) \delta 183.2,142.5,134.9,132.5,130.1,129.4,128.5,128.2,128.1,125.3,121.3,119.9,119.7$, 119.2, 118.4, 117.2, 114.1; MS (ESI): $\mathrm{m} / \mathrm{z}=271.03$ [M] ${ }^{+}$; IR (KBr): 2979, 1708, 1475, 1319, 1295, $1230,1120 \mathrm{~cm}^{-1}$.<smiles>CC(=O)c1c2ccccc2c2ccccn12</smiles>

\section{3kz}

1-(Pyrido[2,1-a]isoindol-6-yl)ethanone (3kz) ${ }^{[6]}$ : yellow solid; $8.8 \mathrm{mg}, 21 \%$ yield; m.p. 135.1136.2 ${ }^{\circ} \mathrm{C} .{ }^{1} \mathrm{H}$ NMR $\left(400 \mathrm{MHz}, \mathrm{CDCl}_{3}\right) \delta 10.66(\mathrm{~d}, J=7.0 \mathrm{~Hz}, 1 \mathrm{H}), 8.22-8.15(\mathrm{~m}, 2 \mathrm{H}), 8.02(\mathrm{~d}, J=$ $8.6 \mathrm{~Hz}, 1 \mathrm{H}), 7.65-7.60(\mathrm{~m}, 1 \mathrm{H}), 7.49(\mathrm{ddd}, J=8.3,7.0,1.0 \mathrm{~Hz}, 1 \mathrm{H}), 7.36-7.30(\mathrm{~m}, 2 \mathrm{H}), 2.85(\mathrm{~s}, 3 \mathrm{H})$; ${ }^{13} \mathrm{C}$ NMR $\left(101 \mathrm{MHz}, \mathrm{CDCl}_{3}\right) \delta 184.1,133.9,131.5,129.2,128.8,124.5,120.8,120.2,119.8,118.8$, 118.3, 117.0, 114.4, 30.4; MS (ESI): m/z = 209.15 [M] $]^{+}$IR (KBr): 2978, 1723, 1469, 1324, 1282, $1233,1115 \mathrm{~cm}^{-1}$.

\section{Controlled experiments for mechanism studies}

(a): The effect of the reaction temperature on a $[4+1]$ cyclative capture access to indolizine.

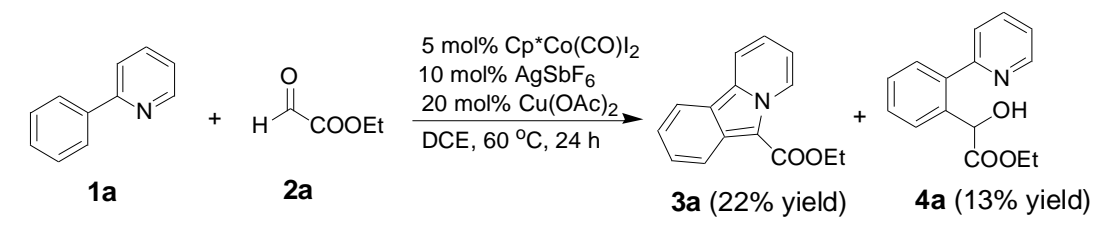

A $10 \mathrm{~mL}$ of reaction tube was charged with 2-phenylpyridine $\mathbf{1}(31.0 \mathrm{mg}, 0.2 \mathrm{mmol})$, $\mathrm{Cp} * \mathrm{Co}(\mathrm{CO}) \mathrm{I}_{2}(4.8 \mathrm{mg}, 5 \mathrm{~mol} \%), \mathrm{AgSbF}_{6}(6.8 \mathrm{mg}, 10 \mathrm{~mol} \%), \mathrm{Cu}(\mathrm{OAc})_{2}(7.2 \mathrm{mg}, 20 \mathrm{~mol} \%)$ and DCE $(1.5 \mathrm{~mL})$ under Ar atmosphere. Then ethyl glyoxylate $2(40.8 \mathrm{mg}, 0.4 \mathrm{mmol})$ in DCE $(0.5 \mathrm{~mL})$ was added in one-pot under Ar and the mixture was stirred at $60{ }^{\circ} \mathrm{C}$ for $24 \mathrm{~h}$. The corresponding reaction mixture was filtered through a pad of Celite, washed with EtOAc and concentrated under reduced pressure. The residue was purified by flash chromatography on silical gel to afford the compounds $3 \mathbf{a}$ (22\% yield) and $\mathbf{4 a}$ (13\% yield).

Ethyl 2-hydroxy-2-(2-(pyridin-2-yl)phenyl)acetate (4a) ${ }^{[7]}$ : yellow solid; m.p. 86.7-87.9 ${ }^{\circ} \mathrm{C} .{ }^{1} \mathrm{H}$ NMR $\left(400 \mathrm{MHz}, \mathrm{CDCl}_{3}\right) \delta 8.62(\mathrm{~d}, J=4.2 \mathrm{~Hz}, 1 \mathrm{H}), 7.84(\mathrm{td}, J=7.8,1.8 \mathrm{~Hz}, 1 \mathrm{H}), 7.59(\mathrm{~d}, J=8.0 \mathrm{~Hz}$, $1 \mathrm{H}), 7.54(\mathrm{dd}, J=5.7,2.9 \mathrm{~Hz}, 1 \mathrm{H}), 7.45(\mathrm{dt}, J=5.7,3.6 \mathrm{~Hz}, 3 \mathrm{H}), 7.32(\mathrm{ddd}, J=7.5,5.0,1.0 \mathrm{~Hz}, 1 \mathrm{H})$, $5.19(\mathrm{~s}, 1 \mathrm{H}), 3.95-3.86(\mathrm{~m}, 2 \mathrm{H}), 0.98(\mathrm{t}, J=7.1 \mathrm{~Hz}, 3 \mathrm{H}),{ }^{13} \mathrm{C} \mathrm{NMR}\left(101 \mathrm{MHz}, \mathrm{CDCl}_{3}\right) \delta 172.7,158.8$, $147.8,138.7,137.7,131.1,130.5,129.3,128.6,123.9,122.5,74.5,60.9,13.9 ; \mathrm{MS}(\mathrm{ESI}): \mathrm{m} / \mathrm{z}=$ $257.09[\mathrm{M}]^{+}$; IR (KBr): 3436, 2974, 1661, 1472, 1345, 1210, 1163, $1036 \mathrm{~cm}^{-1}$. 
(b): The transformation of intermediate $4 \mathrm{a}$ in absence of $\mathrm{Co}$ (III) catalyst.<smiles>CCOC(=O)C(O)c1ccccc1-c1ccccn1</smiles>

$4 a$

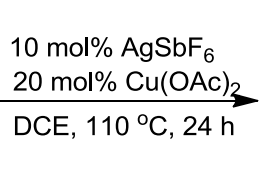<smiles>CCOC(=O)C(O)c1ccccc1-c1ccccn1</smiles>

$4 a(62 \%$ recovery yield)

A $10 \mathrm{~mL}$ of reaction tube was charged with 2-phenylpyridine $4 \mathbf{a}(51.4 \mathrm{mg}, 0.2 \mathrm{mmol}), \mathrm{AgSbF}_{6}$ (6.8 mg, $10 \mathrm{~mol} \%), \mathrm{Cu}(\mathrm{OAc})_{2}(7.2 \mathrm{mg}, 20 \mathrm{~mol} \%)$ and DCE (2 mL) under Ar atmosphere. Then the mixture was stirred at $110{ }^{\circ} \mathrm{C}$ for $24 \mathrm{~h}$. The corresponding reaction mixture was filtered through a pad of Celite, washed with EtOAc and concentrated under reduced pressure. The residue was purified by flash chromatography on silical gel to afford the compounds $\mathbf{3 a}$ in $31 \%$ yield (7.4 $\mathrm{mg})$.

(c): The transformation of intermediate 4 a by using the standard condition.

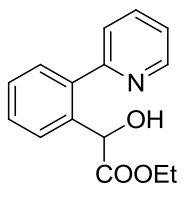

$4 a$

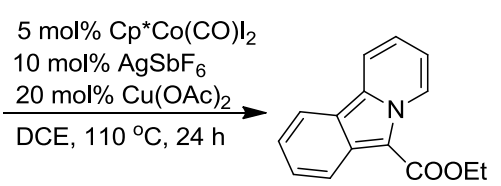

$3 a(92 \%$ yield)

A $10 \mathrm{~mL}$ of reaction tube was charged with 2-phenylpyridine 1 (51.4 mg, $0.2 \mathrm{mmol})$, $\mathrm{Cp} * \mathrm{Co}(\mathrm{CO}) \mathrm{I}_{2}(4.8 \mathrm{mg}, 5 \mathrm{~mol} \%), \mathrm{AgSbF}_{6}(6.8 \mathrm{mg}, 10 \mathrm{~mol} \%), \mathrm{Cu}(\mathrm{OAc})_{2}(7.2 \mathrm{mg}, 20 \mathrm{~mol} \%)$ and DCE $(1.5 \mathrm{~mL})$ under Ar atmosphere. Then the mixture was stirred at $110{ }^{\circ} \mathrm{C}$ for $24 \mathrm{~h}$. The corresponding reaction mixture was filtered through a pad of Celite, washed with EtOAc and concentrated under reduced pressure. The residue was purified by flash chromatography on silical gel to afford the compounds $3 \mathbf{a}$ in $92 \%$ yield $(7.4 \mathrm{mg})$.

(d): H/D exchange of 2-phenylpyridine (1a) by using the standard condition<smiles>c1ccc(-c2ccccn2)cc1</smiles>

$1 \mathrm{a}$

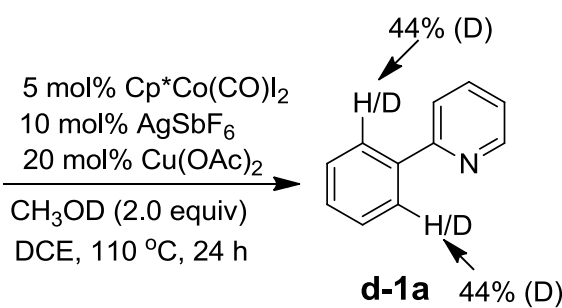

d-1a $44 \%$ (D)

To the solution of 2-phenylpyridine $\mathbf{1}(0.2 \mathrm{mmol})$ in DCE $(1.0 \mathrm{~mL})$ were added $\mathrm{Cp} * \mathrm{Co}(\mathrm{CO}) \mathrm{I}_{2}(4.8$ $\mathrm{mg}, 5 \mathrm{~mol} \%), \mathrm{AgSbF}_{6}(6.8 \mathrm{mg}, 10 \mathrm{~mol} \%), \mathrm{Cu}(\mathrm{OAc})_{2}(7.2 \mathrm{mg}, 20 \mathrm{~mol} \%)$ and $\mathrm{CD}_{3} \mathrm{OD}(2.0$ equiv) under Ar. The reaction mixture was stirred at $110{ }^{\circ} \mathrm{C}$ for $24 \mathrm{~h}$ and then cooled down to room temperature. The corresponding reaction mixture was filtered through a pad of Celite, washed with EtOAc and concentrated under reduced pressure. The residue was purified by flash chromatography on silical gel to afford the desired compound $\boldsymbol{d}$-1a (95\% yield) as white oil. The deuterium incorporation was determined to be $44 \%$ by ${ }^{1} \mathrm{H}$ NMR method (Figure 2). ${ }^{1} \mathrm{H}$ NMR (400 
$\left.\mathrm{MHz} \mathrm{CDCl}_{3}\right) \delta 8.69(\mathrm{~d}, J=4.3 \mathrm{~Hz}, 1.12 \mathrm{H}), 7.99(\mathrm{~d}, J=7.6 \mathrm{~Hz}, 1 \mathrm{H}), 7.72(\mathrm{~d}, J=5.5 \mathrm{~Hz}, 2 \mathrm{H}), 7.46(\mathrm{~s}$, $2 \mathrm{H}), 7.43-7.38(\mathrm{~m}, 1 \mathrm{H}), 7.21(\mathrm{t}, J=5.4 \mathrm{~Hz}, 1 \mathrm{H})$.

\begin{tabular}{|c|c|c|c|}
\hline $\begin{array}{l}g, \infty \\
0 \\
\infty \\
\infty \\
\infty\end{array}$ & $\begin{array}{l}8 \% \\
8 \\
\infty \\
\infty \\
1 \\
1\end{array}$ & 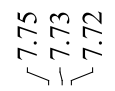 & 守尔永 \\
\hline
\end{tabular}

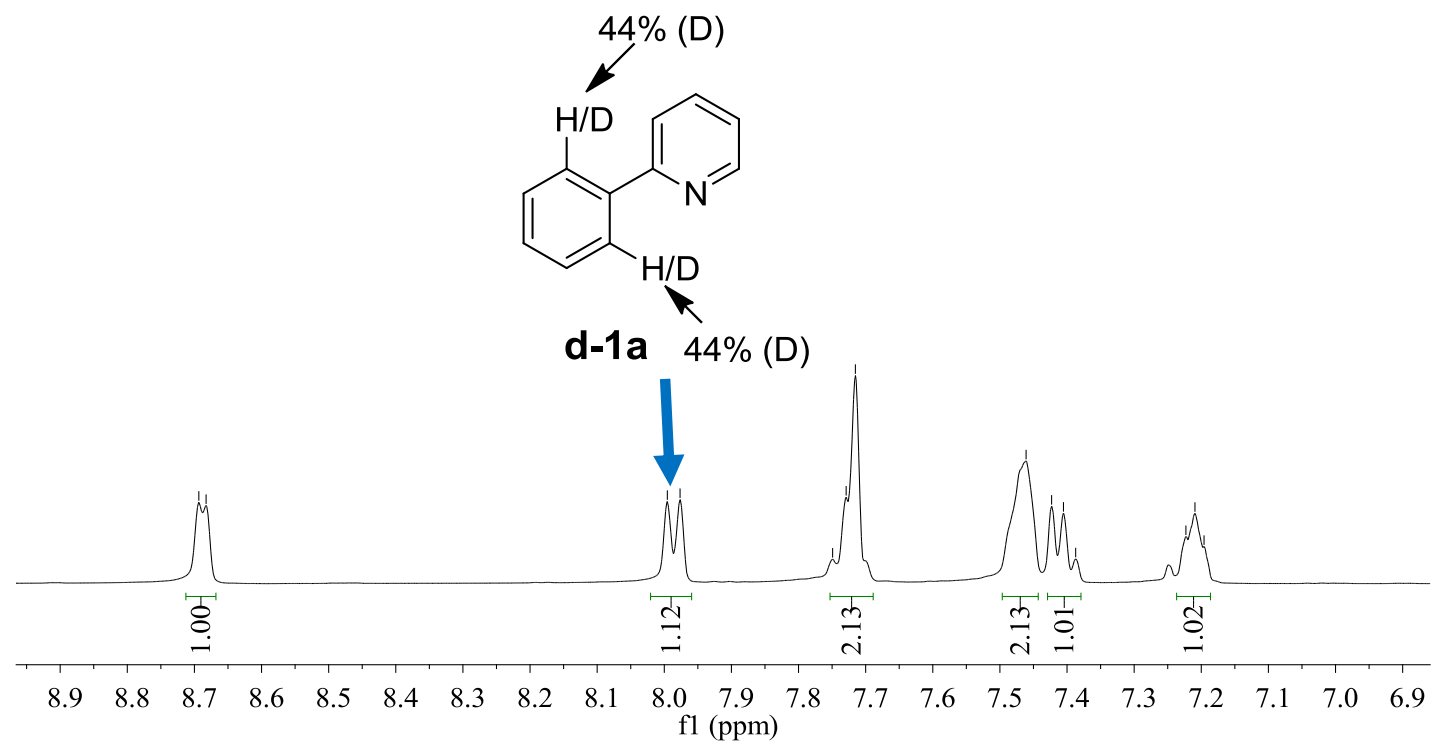

Figure 1. The ${ }^{1} \mathrm{H}$ NMR spectra of $\boldsymbol{d}-\mathbf{1 a}$

(e): The preparation of $\boldsymbol{d}$-1a for KIE test (94\% deuterium incorporation)<smiles>c1ccc(-c2ccccn2)cc1</smiles>

$1 \mathbf{a}$

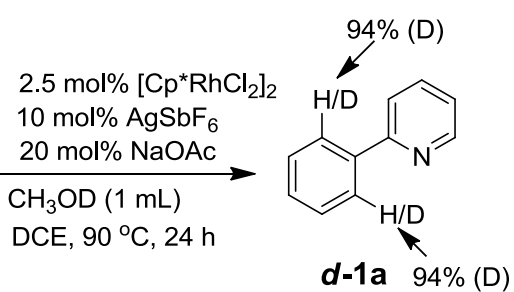

d-1a $94 \%$ (D)

To the solution of 2-phenylpyridine $\mathbf{1}(0.2 \mathrm{mmol})$ in DCE $(1.0 \mathrm{~mL})$ were added [Cp* $\left.\mathrm{RhCl}_{2}\right]_{2}(3.2$ $\mathrm{mg}, 2.5 \mathrm{~mol} \%), \mathrm{AgSbF}_{6}(6.8 \mathrm{mg}, 10 \mathrm{~mol} \%), \mathrm{NaOAc}(3.2 \mathrm{mg}, 20 \mathrm{~mol} \%)$ and $\mathrm{CD}_{3} \mathrm{OD}(1 \mathrm{ml})$ under Ar. The reaction mixture was stirred at $90{ }^{\circ} \mathrm{C}$ for $24 \mathrm{~h}$ and then cooled down to room temperature. The corresponding reaction mixture was filtered through a pad of Celite, washed with EtOAc and concentrated under reduced pressure. The residue was purified by flash chromatography on silical gel to afford the desired compound $\boldsymbol{d}$-1a (97\% yield) as white oil. The deuterium incorporation was determined to be $94 \%$ by ${ }^{1} \mathrm{H}$ NMR method (Figure 2). ${ }^{1} \mathrm{H}$ NMR (400 $\left.\mathrm{MHz}, \mathrm{CDCl}_{3}\right) \delta 8.69(\mathrm{~d}, J=4.6 \mathrm{~Hz}, 1 \mathrm{H}), 7.99(\mathrm{~d}, J=7.7 \mathrm{~Hz}, 0.13 \mathrm{H}), 7.75-7.69(\mathrm{~m}, 2 \mathrm{H}), 7.47(\mathrm{~d}, J=$ $7.1 \mathrm{~Hz}, 2 \mathrm{H}), 7.43-7.38(\mathrm{~m}, 1 \mathrm{H}), 7.23-7.18(\mathrm{~m}, 1 \mathrm{H})$. 


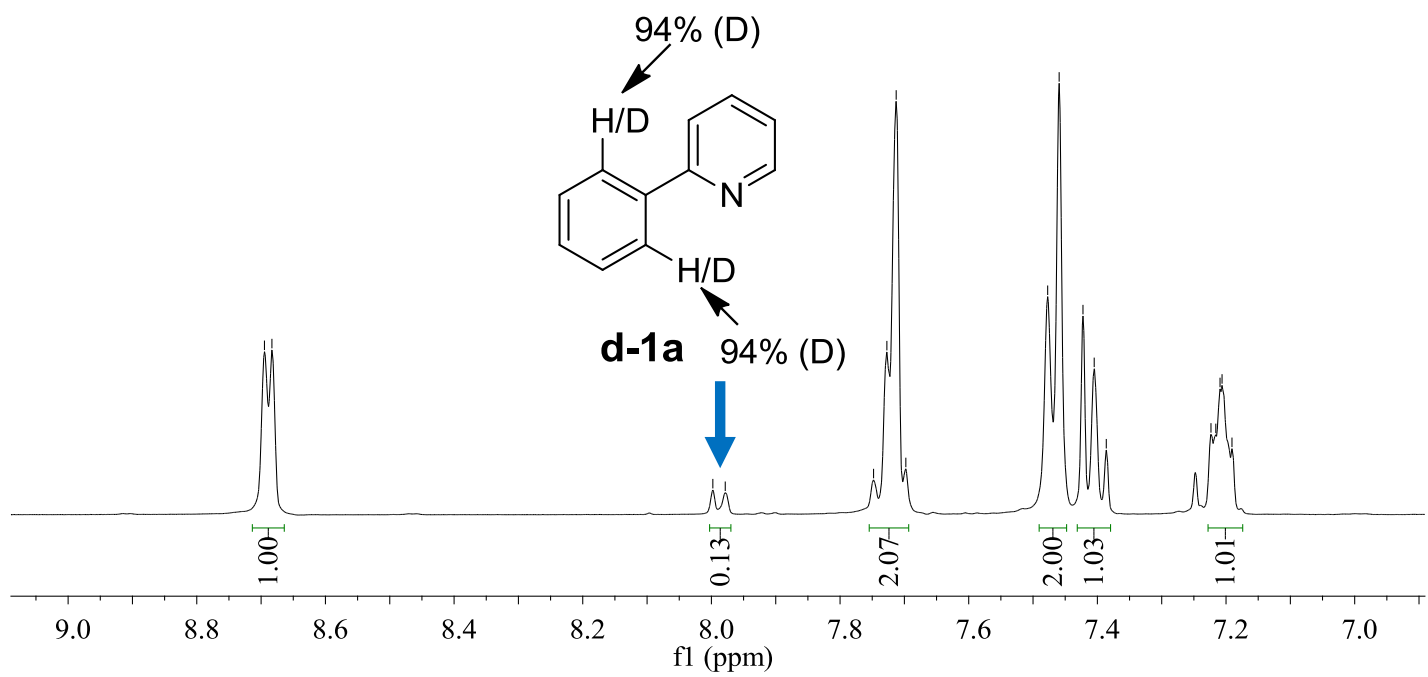

Figure 2. The ${ }^{1} \mathrm{H}$ NMR spectra of $\boldsymbol{d}$-1a

(f): KIE of a [4 + 1] cyclative capture access to indolizines.<smiles>c1ccc2ncccc2c1</smiles>

$1 \mathrm{a}$

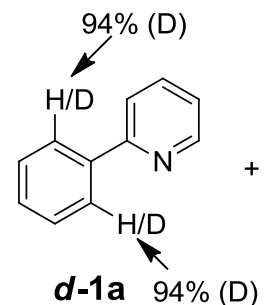<smiles>CCOC(=O)C=O</smiles>

$2 a$

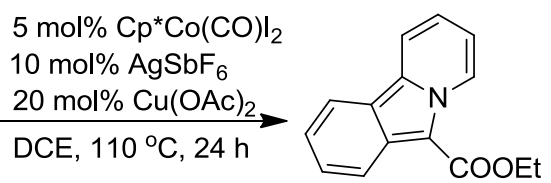

$3 a$

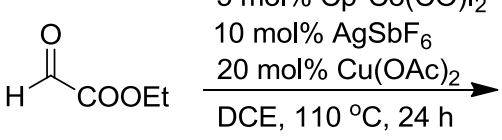

$2 a$

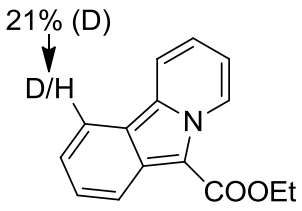

$d-3 a$

A $10 \mathrm{~mL}$ of reaction tube was charged with $N$-carbamoyl indole (1a: $31.0 \mathrm{mg}, 0.2 \mathrm{mmol}$; or $\boldsymbol{d}$-1a: $31.0 \mathrm{mg}, 0.2 \mathrm{mmol}), \mathrm{Cp} * \mathrm{Co}(\mathrm{CO}) \mathrm{I}_{2}(4.8 \mathrm{mg}, 5 \mathrm{~mol} \%), \mathrm{AgSbF}_{6}(6.8 \mathrm{mg}, 10 \mathrm{~mol} \%), \mathrm{Cu}(\mathrm{OAc})_{2}$ $(7.2 \mathrm{mg}, 20 \mathrm{~mol} \%)$ and DCE $(2.0 \mathrm{~mL})$ under Ar atmosphere. Ethyl glyoxylate 2a (40.8 mg, 0.4 $\mathrm{mmol})$ in DCE $(0.5 \mathrm{~mL})$ was then added in one-pot under Ar and the mixture was stirred at $110{ }^{\circ} \mathrm{C}$. Aliquots $(0.5 \mathrm{~mL})$ were extracted at 1 hour intervals for the first 4 hours of the reaction. The corresponding reaction mixture was filtered through a pad of Celite, washed with EtOAc and concentrated under reduced pressure. Then the reaction mixtures were analyzed by ${ }^{1} \mathrm{H}$ NMR spectrum (see Figure 3 and Figure 4). A sample plot of the initial rate data for the reactionn of 
both 1a and $\boldsymbol{d}$-1a was shown in Figure 5. The reaction progress in the early stage (0-4 hours) indicated a kinetic isotope effect (KIE) of 1.13.

$\boldsymbol{d}$-3a: yellow solid; ${ }^{1} \mathrm{H}$ NMR $\left(400 \mathrm{MHz}, \mathrm{CDCl}_{3}\right) \delta 10.03(\mathrm{~d}, J=6.9 \mathrm{~Hz}, 1 \mathrm{H}), 8.32(\mathrm{~d}, J=8.5 \mathrm{~Hz}, 1 \mathrm{H})$, $8.15(\mathrm{dd}, J=11.7,8.4 \mathrm{~Hz}, 1.79 \mathrm{H}), 7.59(\mathrm{dd}, J=8.2,7.1 \mathrm{~Hz}, 1 \mathrm{H}), 7.36-7.25(\mathrm{~m}, 3 \mathrm{H}), 4.55(\mathrm{q}, J=7.1$ $\mathrm{Hz}, 2 \mathrm{H}), 1.56$ (t, $J=7.1 \mathrm{~Hz}, 3 \mathrm{H}) ;{ }^{13} \mathrm{C}$ NMR $\left(101 \mathrm{MHz}, \mathrm{CDCl}_{3}\right) \delta 162.5,132.7,130.9,128.1,127.5$, $121.5,120.7,120.6,119.8,119.4,118.6,117.4,117.2,59.4,14.8$.

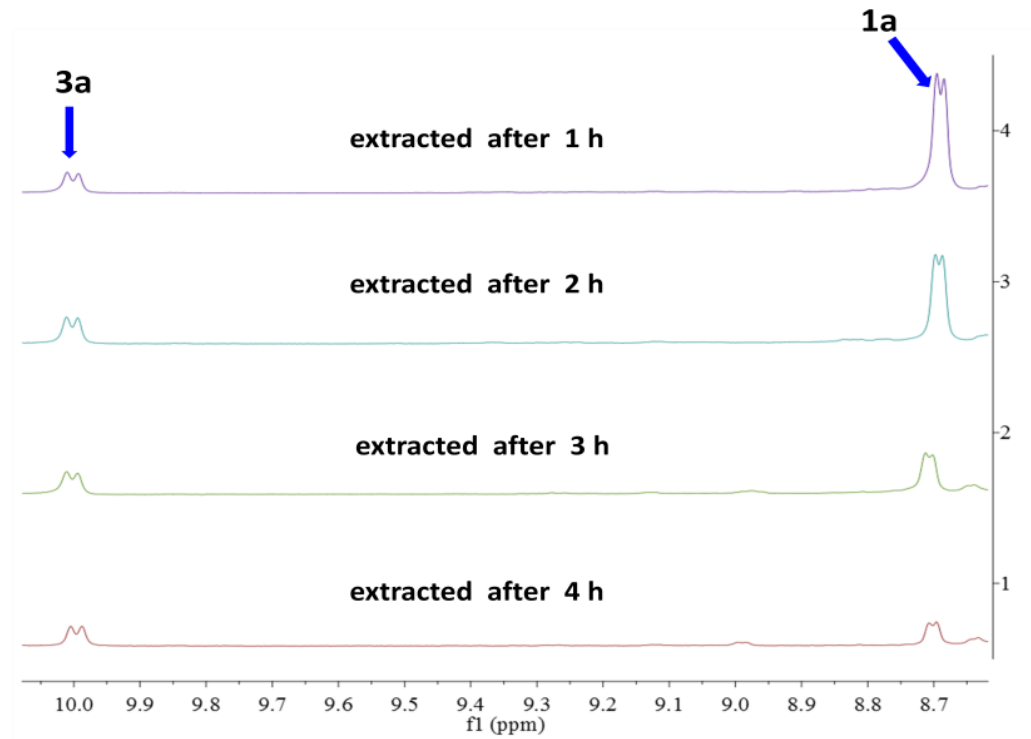

Figure 3, The conversion of $\mathbf{1 a}$ was monitored by ${ }^{1} \mathrm{H}$ NMR method

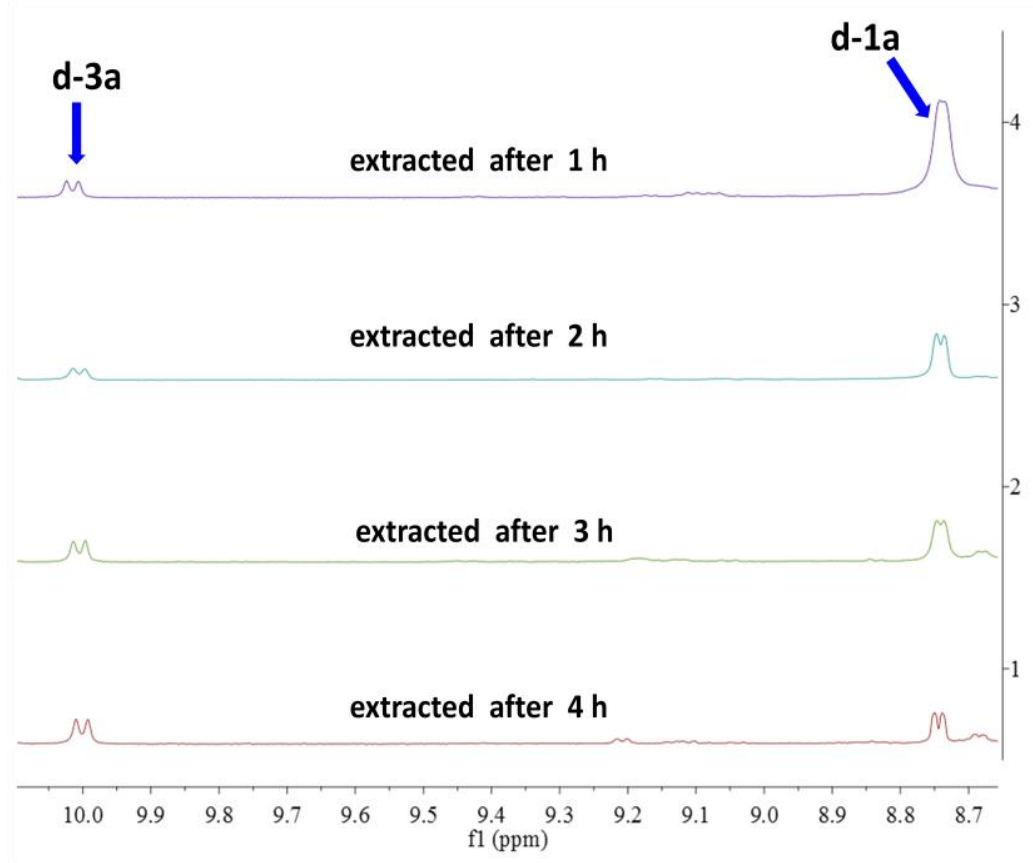

Figure 4. The conversion of $\boldsymbol{d}$-1a was monitored by ${ }^{1} \mathrm{H}$ NMR method 


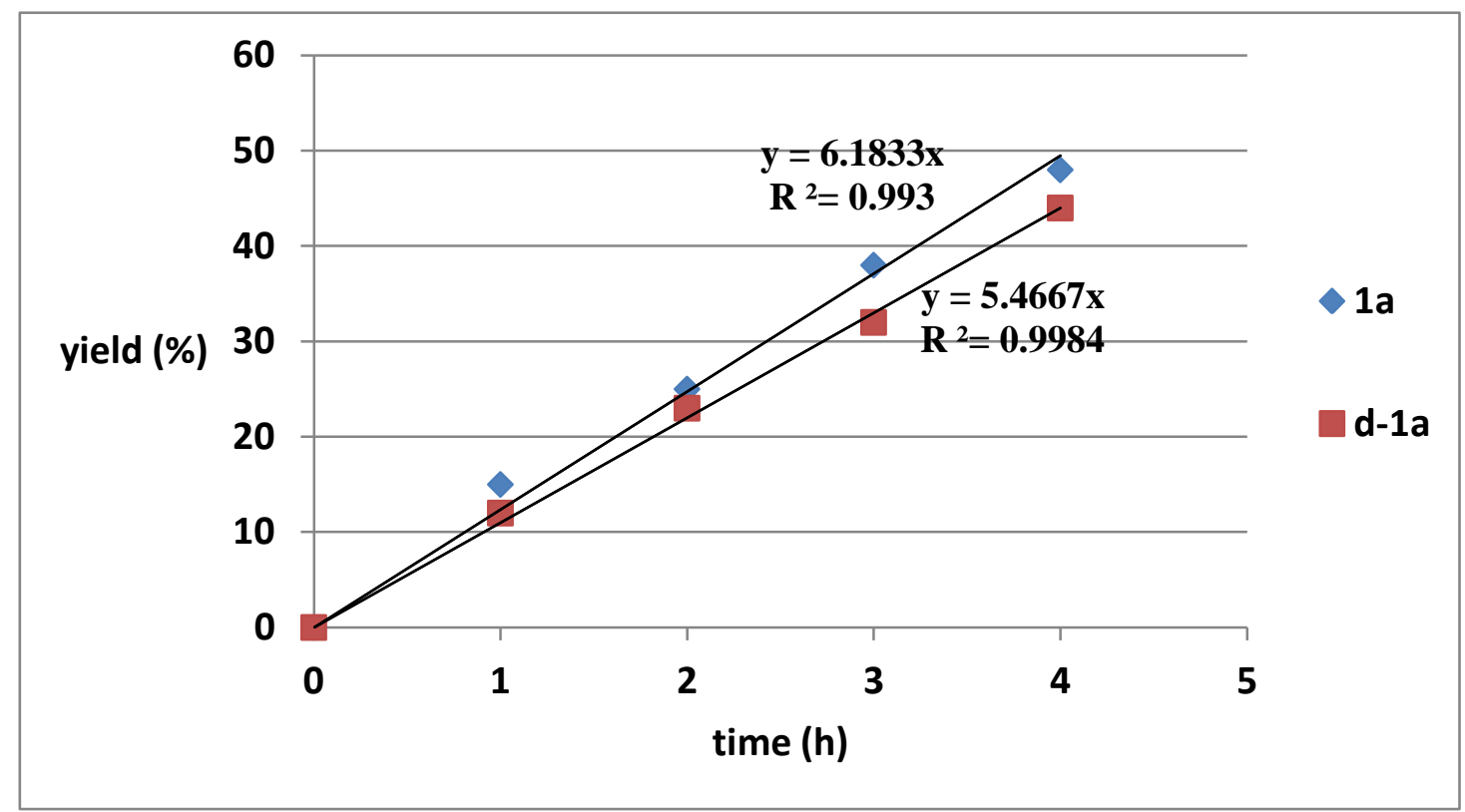

Figure 5. The plot of initial rates for KIE measurements

4. Single crystal structure and crystallographic data for $3 \mathrm{~s}$<smiles>CCOC(=O)c1c2ccccc2c2ccc(Cl)cn12</smiles>

$3 s$

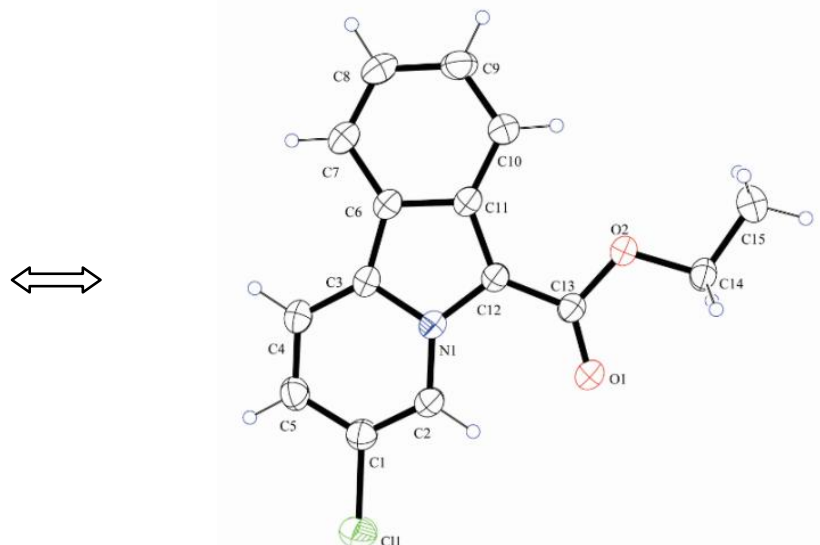

Figure 6. The Single crystal structure of compound 3s (The ellipsoid contour probability level is $\mathbf{3 0 \%}$ )

Table 8. Crystal data and structure refinement for $3 \mathrm{s.}$

Identification code

Empirical formula

Formula weight

Temperature

Wavelength 3s

$\mathrm{C}_{15} \mathrm{H}_{12} \mathrm{ClNO}_{2}$

273.71

571(2) K

$1.54184 \mathrm{~A}$ 
Unit cell dimensions

Volume

Z, Calculated density

Absorption coefficient

$\mathrm{F}(000)$

Crystal size

Theta range for data collection

Limiting indices

Reflections collected / unique

Completeness to theta $=27.51$

Refinement method

Data / restraints / parameters

Goodness-of-fit on $\mathrm{F}^{\wedge} 2$

Final $\mathrm{R}$ indices [I>2sigma(I)]

$\mathrm{R}$ indices (all data)

Largest diff. peak and hole $\mathrm{a}=11.4660(3)$ A alpha $=90 \mathrm{deg}$.

$\mathrm{b}=16.5965(4)$ A $\quad$ beta $=93.942(3) \mathrm{deg}$.

$\mathrm{c}=20.5339(7) \mathrm{A}$ gamma $=90 \mathrm{deg}$.

$3898.3(2) \mathrm{A}^{\wedge} 3$

4, $1.399 \mathrm{Mg} / \mathrm{m}^{\wedge} 3$

$2.579 \mathrm{~mm}^{\wedge}-1$

1704

$0.28 \times 0.26 \times 0.20 \mathrm{~mm}^{3}$

3.43 to $62.80 \mathrm{deg}$.

$-13<=\mathrm{h}<=11,-10<=\mathrm{k}<=18,-19<=\mathrm{l}<=23$

$12776 / 6161[\mathrm{R}(\mathrm{int})=0.0281]$

$98.2 \%$

Full-matrix least-squares on $\mathrm{F}^{\wedge} 2$

$6161 / 0 / 515$

1.037

$\mathrm{R} 1=0.0405, \mathrm{wR} 2=0.0906$

$\mathrm{R} 1=0.0638, \mathrm{wR} 2=0.1033$

0.160 and -0.212 e. $\mathrm{A}^{\wedge}-3$

Table 9. Atomic coordinates $\left(\times 10^{4}\right)$ and equivalent isotropic displacement parameters $\left(\AA^{2} \times\right.$ $10^{3}$ ) for $3 \mathbf{s}$.

$\mathrm{U}(\mathrm{eq})$ is defined as one third of the trace of the orthogonalized $\mathrm{U}^{\mathrm{ij}}$ tensor.

$\begin{array}{llll}\mathrm{x} & \mathrm{z} & \mathrm{U}(\mathrm{eq})\end{array}$

$\begin{array}{lrrrr}\mathrm{C} 1(1) & 6708(1) & 3968(1) & 2317(1) & 86(1) \\ \mathrm{C} 1(2) & 6431(1) & 2538(1) & 724(1) & 84(1) \\ \mathrm{Cl}(3) & 12999(1) & 7521(1) & 1028(1) & 87(1) \\ 0(1) & 10757(1) & 2873(1) & 2624(1) & 59(1) \\ 0(2) & 12619(1) & 3309(1) & 2732(1) & 53(1) \\ 0(3) & 12348(1) & 3174(1) & 1086(1) & 54(1) \\ 0(4) & 10501(1) & 3624(1) & 911(1) & 61(1) \\ 0(5) & 8952(1) & 8607(1) & 733(1) & 59(1) \\ 0(6) & 7097(1) & 8166(1) & 635(1) & 56(1) \\ \mathrm{N}(1) & 10036(2) & 4543(1) & 2532(1) & 43(1) \\ \mathrm{C}(1) & 7998(2) & 4518(1) & 2379(1) & 55(1)\end{array}$




\begin{tabular}{|c|c|c|c|c|}
\hline $\mathrm{C}(2)$ & $9014(2)$ & $4115(1)$ & $2472(1)$ & $51(1)$ \\
\hline$C(3)$ & $10017(2)$ & $5385(1)$ & $2500(1)$ & $45(1)$ \\
\hline C (4) & $8958(2)$ & $5784(1)$ & $2404(1)$ & $53(1)$ \\
\hline$C(5)$ & $7936(2)$ & $5358(1)$ & $2342(1)$ & $57(1)$ \\
\hline$C(6)$ & $11187(2)$ & $5649(1)$ & $2578(1)$ & $45(1)$ \\
\hline$C(7)$ & $11656(2)$ & $6431(1)$ & $2582(1)$ & $56(1)$ \\
\hline $\mathrm{C}(8)$ & $12839(2)$ & $6516(2)$ & $2658(1)$ & $65(1)$ \\
\hline $\mathrm{C}(9)$ & $13565(2)$ & $5836(2)$ & $2729(1)$ & $63(1)$ \\
\hline$C(10)$ & $13132(2)$ & $5069(1)$ & $2729(1)$ & $55(1)$ \\
\hline$C(11)$ & $11911(2)$ & $4961(1)$ & $2652(1)$ & $45(1)$ \\
\hline C (12) & $11184(2)$ & $4272(1)$ & $2623(1)$ & $44(1)$ \\
\hline C (13) & $11453(2)$ & 3425 (1) & 2657 (1) & $45(1)$ \\
\hline C (14) & $12997(2)$ & $2480(1)$ & 2747 (1) & $52(1)$ \\
\hline$C(15)$ & $14293(2)$ & $2473(2)$ & $2835(2)$ & $82(1)$ \\
\hline$C(16)$ & $7712(2)$ & $1983(2)$ & $735(1)$ & $58(1)$ \\
\hline$C(17)$ & $8741(2)$ & 2387 (1) & $818(1)$ & $53(1)$ \\
\hline C (18) & $9741(2)$ & $1112(1)$ & $811(1)$ & $48(1)$ \\
\hline$C(19)$ & $8668(2)$ & $721(1)$ & $717(1)$ & $58(1)$ \\
\hline$C(20)$ & 7657 (2) & $1148(2)$ & $674(1)$ & $63(1)$ \\
\hline$C(21)$ & $10907(2)$ & $842(1)$ & $897(1)$ & $47(1)$ \\
\hline$C(22)$ & $11373(2)$ & $59(1)$ & $912(1)$ & $58(1)$ \\
\hline C (23) & $12551(2)$ & $-26(2)$ & $1020(1)$ & $67(1)$ \\
\hline$C(24)$ & $13277(2)$ & $646(2)$ & $1109(1)$ & $64(1)$ \\
\hline$C(25)$ & $12853(2)$ & $1419(2)$ & $1085(1)$ & $56(1)$ \\
\hline$C(26)$ & $11635(2)$ & $1532(1)$ & $982(1)$ & $47(1)$ \\
\hline $\mathrm{C}(27)$ & $10912(2)$ & $2220(1)$ & $945(1)$ & $46(1)$ \\
\hline$C(28)$ & $11190(2)$ & 3064 (1) & $978(1)$ & $47(1)$ \\
\hline C (29) & $12754(2)$ & 3997 (1) & $1093(1)$ & $52(1)$ \\
\hline$C(30)$ & $14045(2)$ & $3976(2)$ & $1237(2)$ & $74(1)$ \\
\hline$C(31)$ & $11726(2)$ & $6963(2)$ & $911(1)$ & $58(1)$ \\
\hline C (32) & $10699(2)$ & $7365(1)$ & $848(1)$ & $52(1)$ \\
\hline C (33) & $9705(2)$ & $6093(1)$ & $756(1)$ & $47(1)$ \\
\hline C (34) & $10777(2)$ & $5700(2)$ & $817(1)$ & $59(1)$ \\
\hline C (35) & $11788(2)$ & $6124(2)$ & $894(1)$ & $66(1)$ \\
\hline C (36) & $8535(2)$ & $5824(1)$ & $684(1)$ & $46(1)$ \\
\hline C (37) & $8056(2)$ & $5047(1)$ & $660(1)$ & $56(1)$ \\
\hline C (38) & $6873(2)$ & $4965(2)$ & $602(1)$ & $65(1)$ \\
\hline C (39) & $6148(2)$ & $5644(2)$ & $570(1)$ & $64(1)$ \\
\hline
\end{tabular}




$\begin{array}{lllll}\text { C(40) } & 6589(2) & 6414(1) & 588(1) & 56(1) \\ \text { C (41) } & 7806(2) & 6517(1) & 649(1) & 45(1) \\ \text { C (42) } & 8534(2) & 7207(1) & 698(1) & 45(1) \\ \text { C (43) } & 8257(2) & 8052(1) & 691(1) & 47(1) \\ \text { C (44) } & 6707(2) & 8992(1) & 624(1) & 56(1) \\ \text { C (45) } & 5409(2) & 8981(2) & 539(2) & 88(1) \\ \text { N(3) } & 9681(2) & 6934(1) & 766(1) & 44(1) \\ \text { N(2) } & 9760(2) & 1954(1) & 851(1) & 46(1)\end{array}$

Table 10. Bond lengths $[A]$ and angles $[\mathrm{deg}]$ for $3 \mathrm{~s}$.

\begin{tabular}{|c|c|}
\hline $\mathrm{Cl}(1)-\mathrm{C}(1)$ & 1. $735(2)$ \\
\hline $\mathrm{Cl}(2)-\mathrm{C}(16)$ & 1. $732(3)$ \\
\hline $\mathrm{Cl}(3)-\mathrm{C}(31)$ & 1. $732(2)$ \\
\hline $0(1)-C(13)$ & $1.213(2)$ \\
\hline $0(2)-C(13)$ & $1.349(3)$ \\
\hline $0(2)-C(14)$ & 1. $441(3)$ \\
\hline $0(3)-C(28)$ & $1.343(3)$ \\
\hline $0(3)-C(29)$ & 1. $443(3)$ \\
\hline $0(4)-C(28)$ & 1. $223(3)$ \\
\hline $0(5)-C(43)$ & 1. $218(3)$ \\
\hline $0(6)-C(43)$ & 1. 341 (3) \\
\hline $0(6)-C(44)$ & 1. $441(3)$ \\
\hline $\mathrm{N}(1)-\mathrm{C}(2)$ & $1.369(3)$ \\
\hline$N(1)-C(12)$ & 1. $392(3)$ \\
\hline $\mathrm{N}(1)-\mathrm{C}(3)$ & $1.398(3)$ \\
\hline$C(1)-C(2)$ & $1.345(3)$ \\
\hline$C(1)-C(5)$ & $1.397(3)$ \\
\hline $\mathrm{C}(2)-\mathrm{H}(2 \mathrm{~A})$ & 0.9300 \\
\hline$C(3)-C(4)$ & 1. $385(3)$ \\
\hline$C(3)-C(6)$ & $1.411(3)$ \\
\hline$C(4)-C(5)$ & $1.367(3)$ \\
\hline $\mathrm{C}(4)-\mathrm{H}(4 \mathrm{~A})$ & 0.9300 \\
\hline $\mathrm{C}(5)-\mathrm{H}(5 \mathrm{~A})$ & 0.9300 \\
\hline$C(6)-C(7)$ & 1. $404(3)$ \\
\hline$C(6)-C(11)$ & 1. $415(3)$ \\
\hline$C(7)-C(8)$ & 1. $362(4)$ \\
\hline $\mathrm{C}(7)-\mathrm{H}(7 \mathrm{~A})$ & 0.9300 \\
\hline
\end{tabular}




\begin{tabular}{|c|c|}
\hline C (8)-C (9) & 1. $405(4)$ \\
\hline $\mathrm{C}(8)-\mathrm{H}(8 \mathrm{~A})$ & 0.9300 \\
\hline$C(9)-C(10)$ & $1.367(3)$ \\
\hline $\mathrm{C}(9)-\mathrm{H}(9 \mathrm{~A})$ & 0.9300 \\
\hline$C(10)-C(11)$ & 1. $409(3)$ \\
\hline $\mathrm{C}(10)-\mathrm{H}(10 \mathrm{~A})$ & 0.9300 \\
\hline$C(11)-C(12)$ & $1.414(3)$ \\
\hline$C(12)-C(13)$ & 1. $439(3)$ \\
\hline$C(14)-C(15)$ & 1. $484(4)$ \\
\hline $\mathrm{C}(14)-\mathrm{H}(14 \mathrm{~A})$ & 0.9700 \\
\hline $\mathrm{C}(14)-\mathrm{H}(14 \mathrm{~B})$ & 0.9700 \\
\hline $\mathrm{C}(15)-\mathrm{H}(15 \mathrm{~A})$ & 0.9600 \\
\hline $\mathrm{C}(15)-\mathrm{H}(15 \mathrm{~B})$ & 0.9600 \\
\hline $\mathrm{C}(15)-\mathrm{H}(15 \mathrm{C})$ & 0.9600 \\
\hline$C(16)-C(17)$ & 1. $357(3)$ \\
\hline$C(16)-C(20)$ & $1.393(3)$ \\
\hline $\mathrm{C}(17)-\mathrm{N}(2)$ & 1. $369(3)$ \\
\hline $\mathrm{C}(17)-\mathrm{H}(17 \mathrm{~A})$ & 0.9300 \\
\hline$C(18)-C(19)$ & 1. $392(3)$ \\
\hline $\mathrm{C}(18)-\mathrm{N}(2)$ & 1. $400(3)$ \\
\hline$C(18)-C(21)$ & 1. $410(3)$ \\
\hline$C(19)-C(20)$ & 1. $357(4)$ \\
\hline$C(19)-H(19 A)$ & 0.9300 \\
\hline $\mathrm{C}(20)-\mathrm{H}(20 \mathrm{~A})$ & 0.9300 \\
\hline$C(21)-C(22)$ & $1.404(3)$ \\
\hline$C(21)-C(26)$ & 1. $422(3)$ \\
\hline$C(22)-C(23)$ & 1. $361(4)$ \\
\hline $\mathrm{C}(22)-\mathrm{H}(22 \mathrm{~A})$ & 0.9300 \\
\hline$C(23)-C(24)$ & $1.397(4)$ \\
\hline $\mathrm{C}(23)-\mathrm{H}(23 \mathrm{~A})$ & 0.9300 \\
\hline$C(24)-C(25)$ & 1. $371(3)$ \\
\hline $\mathrm{C}(24)-\mathrm{H}(24 \mathrm{~A})$ & 0.9300 \\
\hline$C(25)-C(26)$ & 1. $410(3)$ \\
\hline $\mathrm{C}(25)-\mathrm{H}(25 \mathrm{~A})$ & 0.9300 \\
\hline$C(26)-C(27)$ & 1. $410(3)$ \\
\hline $\mathrm{C}(27)-\mathrm{N}(2)$ & 1. $393(3)$ \\
\hline$C(27)-C(28)$ & 1. $437(3)$ \\
\hline $\mathrm{C}(29)-\mathrm{C}(30)$ & 1. $489(3)$ \\
\hline
\end{tabular}




\begin{tabular}{|c|c|}
\hline $\mathrm{C}(29)-\mathrm{H}(29 \mathrm{~A})$ & 0.9700 \\
\hline C (29) -H (29B) & 0.9700 \\
\hline $\mathrm{C}(30)-\mathrm{H}(30 \mathrm{~A})$ & 0.9600 \\
\hline $\mathrm{C}(30)-\mathrm{H}(30 \mathrm{~B})$ & 0.9600 \\
\hline $\mathrm{C}(30)-\mathrm{H}(30 \mathrm{C})$ & 0.9600 \\
\hline$C(31)-C(32)$ & 1. $351(3)$ \\
\hline$C(31)-C(35)$ & $1.395(4)$ \\
\hline $\mathrm{C}(32)-\mathrm{N}(3)$ & $1.370(3)$ \\
\hline $\mathrm{C}(32)-\mathrm{H}(32 \mathrm{~A})$ & 0.9300 \\
\hline$C(33)-C(34)$ & $1.390(3)$ \\
\hline $\mathrm{C}(33)-\mathrm{N}(3)$ & 1. $397(3)$ \\
\hline$C(33)-C(36)$ & 1. $412(3)$ \\
\hline$C(34)-C(35)$ & $1.357(4)$ \\
\hline $\mathrm{C}(34)-\mathrm{H}(34 \mathrm{~A})$ & 0.9300 \\
\hline $\mathrm{C}(35)-\mathrm{H}(35 \mathrm{~A})$ & 0.9300 \\
\hline$C(36)-C(37)$ & 1. $401(3)$ \\
\hline$C(36)-C(41)$ & 1. $420(3)$ \\
\hline$C(37)-C(38)$ & $1.360(4)$ \\
\hline $\mathrm{C}(37)-\mathrm{H}(37 \mathrm{~A})$ & 0.9300 \\
\hline$C(38)-C(39)$ & 1. $400(4)$ \\
\hline $\mathrm{C}(38)-\mathrm{H}(38 \mathrm{~A})$ & 0.9300 \\
\hline$C(39)-C(40)$ & 1. $374(3)$ \\
\hline $\mathrm{C}(39)-\mathrm{H}(39 \mathrm{~A})$ & 0.9300 \\
\hline$C(40)-C(41)$ & 1. $403(3)$ \\
\hline $\mathrm{C}(40)-\mathrm{H}(40 \mathrm{~A})$ & 0.9300 \\
\hline$C(41)-C(42)$ & 1. $417(3)$ \\
\hline $\mathrm{C}(42)-\mathrm{N}(3)$ & $1.389(3)$ \\
\hline$C(42)-C(43)$ & $1.438(3)$ \\
\hline$C(44)-C(45)$ & 1. $487(4)$ \\
\hline $\mathrm{C}(44)-\mathrm{H}(44 \mathrm{~A})$ & 0.9700 \\
\hline $\mathrm{C}(44)-\mathrm{H}(44 \mathrm{~B})$ & 0.9700 \\
\hline $\mathrm{C}(45)-\mathrm{H}(45 \mathrm{~A})$ & 0.9600 \\
\hline $\mathrm{C}(45)-\mathrm{H}(45 \mathrm{~B})$ & 0.9600 \\
\hline $\mathrm{C}(45)-\mathrm{H}(45 \mathrm{C})$ & 0.9600 \\
\hline$C(13)-0(2)-C(14)$ & $115.68(16)$ \\
\hline$C(28)-0(3)-C(29)$ & $116.46(17)$ \\
\hline$C(43)-0(6)-C(44)$ & $116.13(17)$ \\
\hline
\end{tabular}




\begin{tabular}{|c|c|}
\hline $\mathrm{C}(2)-\mathrm{N}(1)-\mathrm{C}(12)$ & 129. $77(18)$ \\
\hline$C(2)-N(1)-C(3)$ & $120.27(18)$ \\
\hline $\mathrm{C}(12)-\mathrm{N}(1)-\mathrm{C}(3)$ & $109.96(17)$ \\
\hline$C(2)-C(1)-C(5)$ & $123.0(2)$ \\
\hline $\mathrm{C}(2)-\mathrm{C}(1)-\mathrm{Cl}(1)$ & 118. 28(18) \\
\hline $\mathrm{C}(5)-\mathrm{C}(1)-\mathrm{Cl}(1)$ & 118. $74(18)$ \\
\hline $\mathrm{C}(1)-\mathrm{C}(2)-\mathrm{N}(1)$ & $118.8(2)$ \\
\hline $\mathrm{C}(1)-\mathrm{C}(2)-\mathrm{H}(2 \mathrm{~A})$ & 120.6 \\
\hline $\mathrm{N}(1)-\mathrm{C}(2)-\mathrm{H}(2 \mathrm{~A})$ & 120.6 \\
\hline $\mathrm{C}(4)-\mathrm{C}(3)-\mathrm{N}(1)$ & $119.6(2)$ \\
\hline$C(4)-C(3)-C(6)$ & 133. $3(2)$ \\
\hline$N(1)-C(3)-C(6)$ & $107.07(18)$ \\
\hline$C(5)-C(4)-C(3)$ & $120.3(2)$ \\
\hline $\mathrm{C}(5)-\mathrm{C}(4)-\mathrm{H}(4 \mathrm{~A})$ & 119.9 \\
\hline $\mathrm{C}(3)-\mathrm{C}(4)-\mathrm{H}(4 \mathrm{~A})$ & 119.9 \\
\hline$C(4)-C(5)-C(1)$ & $118.1(2)$ \\
\hline$C(4)-C(5)-H(5 A)$ & 121.0 \\
\hline $\mathrm{C}(1)-\mathrm{C}(5)-\mathrm{H}(5 \mathrm{~A})$ & 121.0 \\
\hline$C(7)-C(6)-C(3)$ & $130.5(2)$ \\
\hline$C(7)-C(6)-C(11)$ & $121.6(2)$ \\
\hline$C(3)-C(6)-C(11)$ & $107.93(17)$ \\
\hline$C(8)-C(7)-C(6)$ & $118.3(2)$ \\
\hline $\mathrm{C}(8)-\mathrm{C}(7)-\mathrm{H}(7 \mathrm{~A})$ & 120.8 \\
\hline $\mathrm{C}(6)-\mathrm{C}(7)-\mathrm{H}(7 \mathrm{~A})$ & 120.8 \\
\hline$C(7)-C(8)-C(9)$ & $120.4(2)$ \\
\hline $\mathrm{C}(7)-\mathrm{C}(8)-\mathrm{H}(8 \mathrm{~A})$ & 119.8 \\
\hline $\mathrm{C}(9)-\mathrm{C}(8)-\mathrm{H}(8 \mathrm{~A})$ & 119.8 \\
\hline$C(10)-C(9)-C(8)$ & $122.4(2)$ \\
\hline $\mathrm{C}(10)-\mathrm{C}(9)-\mathrm{H}(9 \mathrm{~A})$ & 118.8 \\
\hline $\mathrm{C}(8)-\mathrm{C}(9)-\mathrm{H}(9 \mathrm{~A})$ & 118.8 \\
\hline$C(9)-C(10)-C(11)$ & $118.5(2)$ \\
\hline $\mathrm{C}(9)-\mathrm{C}(10)-\mathrm{H}(10 \mathrm{~A})$ & 120.8 \\
\hline $\mathrm{C}(11)-\mathrm{C}(10)-\mathrm{H}(10 \mathrm{~A})$ & 120.8 \\
\hline$C(10)-C(11)-C(12)$ & $133.3(2)$ \\
\hline$C(10)-C(11)-C(6)$ & $118.70(19)$ \\
\hline$C(12)-C(11)-C(6)$ & $107.96(18)$ \\
\hline$N(1)-C(12)-C(11)$ & 107. $07(17)$ \\
\hline$N(1)-C(12)-C(13)$ & $121.33(18)$ \\
\hline
\end{tabular}




\begin{tabular}{|c|c|}
\hline$C(11)-C(12)-C(13)$ & $131.58(19)$ \\
\hline $0(1)-C(13)-0(2)$ & $122.73(18)$ \\
\hline $0(1)-C(13)-C(12)$ & 126. $64(19)$ \\
\hline $0(2)-C(13)-C(12)$ & $110.63(18)$ \\
\hline $0(2)-C(14)-C(15)$ & $107.92(19)$ \\
\hline $0(2)-\mathrm{C}(14)-\mathrm{H}(14 \mathrm{~A})$ & 110.1 \\
\hline $\mathrm{C}(15)-\mathrm{C}(14)-\mathrm{H}(14 \mathrm{~A})$ & 110.1 \\
\hline $0(2)-\mathrm{C}(14)-\mathrm{H}(14 \mathrm{~B})$ & 110.1 \\
\hline $\mathrm{C}(15)-\mathrm{C}(14)-\mathrm{H}(14 \mathrm{~B})$ & 110.1 \\
\hline $\mathrm{H}(14 \mathrm{~A})-\mathrm{C}(14)-\mathrm{H}(14 \mathrm{~B})$ & 108.4 \\
\hline $\mathrm{C}(14)-\mathrm{C}(15)-\mathrm{H}(15 \mathrm{~A})$ & 109.5 \\
\hline $\mathrm{C}(14)-\mathrm{C}(15)-\mathrm{H}(15 \mathrm{~B})$ & 109.5 \\
\hline $\mathrm{H}(15 \mathrm{~A})-\mathrm{C}(15)-\mathrm{H}(15 \mathrm{~B})$ & 109.5 \\
\hline $\mathrm{C}(14)-\mathrm{C}(15)-\mathrm{H}(15 \mathrm{C})$ & 109.5 \\
\hline $\mathrm{H}(15 \mathrm{~A})-\mathrm{C}(15)-\mathrm{H}(15 \mathrm{C})$ & 109.5 \\
\hline $\mathrm{H}(15 \mathrm{~B})-\mathrm{C}(15)-\mathrm{H}(15 \mathrm{C})$ & 109.5 \\
\hline$C(17)-C(16)-C(20)$ & $122.5(2)$ \\
\hline$C(17)-C(16)-C 1(2)$ & $117.88(19)$ \\
\hline$C(20)-C(16)-C 1(2)$ & 119.63(19) \\
\hline $\mathrm{C}(16)-\mathrm{C}(17)-\mathrm{N}(2)$ & $118.6(2)$ \\
\hline $\mathrm{C}(16)-\mathrm{C}(17)-\mathrm{H}(17 \mathrm{~A})$ & 120.7 \\
\hline $\mathrm{N}(2)-\mathrm{C}(17)-\mathrm{H}(17 \mathrm{~A})$ & 120.7 \\
\hline $\mathrm{C}(19)-\mathrm{C}(18)-\mathrm{N}(2)$ & $118.9(2)$ \\
\hline$C(19)-C(18)-C(21)$ & $133.6(2)$ \\
\hline$N(2)-C(18)-C(21)$ & 107. $41(19)$ \\
\hline$C(20)-C(19)-C(18)$ & $120.6(2)$ \\
\hline $\mathrm{C}(20)-\mathrm{C}(19)-\mathrm{H}(19 \mathrm{~A})$ & 119.7 \\
\hline $\mathrm{C}(18)-\mathrm{C}(19)-\mathrm{H}(19 \mathrm{~A})$ & 119.7 \\
\hline$C(19)-C(20)-C(16)$ & $118.7(2)$ \\
\hline $\mathrm{C}(19)-\mathrm{C}(20)-\mathrm{H}(20 \mathrm{~A})$ & 120.7 \\
\hline $\mathrm{C}(16)-\mathrm{C}(20)-\mathrm{H}(20 \mathrm{~A})$ & 120.7 \\
\hline$C(22)-C(21)-C(18)$ & $130.8(2)$ \\
\hline$C(22)-C(21)-C(26)$ & $121.5(2)$ \\
\hline$C(18)-C(21)-C(26)$ & $107.64(18)$ \\
\hline$C(23)-C(22)-C(21)$ & $118.2(2)$ \\
\hline $\mathrm{C}(23)-\mathrm{C}(22)-\mathrm{H}(22 \mathrm{~A})$ & 120.9 \\
\hline $\mathrm{C}(21)-\mathrm{C}(22)-\mathrm{H}(22 \mathrm{~A})$ & 120.9 \\
\hline$C(22)-C(23)-C(24)$ & $120.9(2)$ \\
\hline
\end{tabular}




\begin{tabular}{|c|c|}
\hline $\mathrm{C}(22)-\mathrm{C}(23)-\mathrm{H}(23 \mathrm{~A})$ & 119.5 \\
\hline $\mathrm{C}(24)-\mathrm{C}(23)-\mathrm{H}(23 \mathrm{~A})$ & 119.5 \\
\hline$C(25)-C(24)-C(23)$ & $122.4(2)$ \\
\hline $\mathrm{C}(25)-\mathrm{C}(24)-\mathrm{H}(24 \mathrm{~A})$ & 118.8 \\
\hline $\mathrm{C}(23)-\mathrm{C}(24)-\mathrm{H}(24 \mathrm{~A})$ & 118.8 \\
\hline$C(24)-C(25)-C(26)$ & $118.4(2)$ \\
\hline $\mathrm{C}(24)-\mathrm{C}(25)-\mathrm{H}(25 \mathrm{~A})$ & 120.8 \\
\hline $\mathrm{C}(26)-\mathrm{C}(25)-\mathrm{H}(25 \mathrm{~A})$ & 120.8 \\
\hline$C(27)-C(26)-C(25)$ & $133.6(2)$ \\
\hline$C(27)-C(26)-C(21)$ & 107. $88(19)$ \\
\hline$C(25)-C(26)-C(21)$ & $118.55(19)$ \\
\hline $\mathrm{N}(2)-\mathrm{C}(27)-\mathrm{C}(26)$ & $107.47(17)$ \\
\hline$N(2)-C(27)-C(28)$ & $121.4(2)$ \\
\hline$C(26)-C(27)-C(28)$ & 131. 2(2) \\
\hline $0(4)-C(28)-0(3)$ & $122.58(19)$ \\
\hline $0(4)-C(28)-C(27)$ & 126. $6(2)$ \\
\hline $0(3)-C(28)-C(27)$ & 110. $77(19)$ \\
\hline $0(3)-C(29)-C(30)$ & 107. $16(19)$ \\
\hline $0(3)-C(29)-H(29 A)$ & 110.3 \\
\hline $\mathrm{C}(30)-\mathrm{C}(29)-\mathrm{H}(29 \mathrm{~A})$ & 110.3 \\
\hline $0(3)-C(29)-H(29 B)$ & 110.3 \\
\hline$C(30)-C(29)-H(29 B)$ & 110.3 \\
\hline$H(29 A)-C(29)-H(29 B)$ & 108.5 \\
\hline $\mathrm{C}(29)-\mathrm{C}(30)-\mathrm{H}(30 \mathrm{~A})$ & 109.5 \\
\hline $\mathrm{C}(29)-\mathrm{C}(30)-\mathrm{H}(30 \mathrm{~B})$ & 109.5 \\
\hline $\mathrm{H}(30 \mathrm{~A})-\mathrm{C}(30)-\mathrm{H}(30 \mathrm{~B})$ & 109.5 \\
\hline $\mathrm{C}(29)-\mathrm{C}(30)-\mathrm{H}(30 \mathrm{C})$ & 109.5 \\
\hline $\mathrm{H}(30 \mathrm{~A})-\mathrm{C}(30)-\mathrm{H}(30 \mathrm{C})$ & 109.5 \\
\hline $\mathrm{H}(30 \mathrm{~B})-\mathrm{C}(30)-\mathrm{H}(30 \mathrm{C})$ & 109.5 \\
\hline$C(32)-C(31)-C(35)$ & $122.5(2)$ \\
\hline$C(32)-C(31)-C 1(3)$ & $118.0(2)$ \\
\hline$C(35)-C(31)-C 1(3)$ & $119.55(19)$ \\
\hline $\mathrm{C}(31)-\mathrm{C}(32)-\mathrm{N}(3)$ & $118.9(2)$ \\
\hline $\mathrm{C}(31)-\mathrm{C}(32)-\mathrm{H}(32 \mathrm{~A})$ & 120.6 \\
\hline $\mathrm{N}(3)-\mathrm{C}(32)-\mathrm{H}(32 \mathrm{~A})$ & 120.6 \\
\hline $\mathrm{C}(34)-\mathrm{C}(33)-\mathrm{N}(3)$ & $119.1(2)$ \\
\hline$C(34)-C(33)-C(36)$ & 133. 6(2) \\
\hline$N(3)-C(33)-C(36)$ & $107.30(19)$ \\
\hline
\end{tabular}




\begin{tabular}{|c|c|}
\hline$C(35)-C(34)-C(33)$ & $120.7(2)$ \\
\hline $\mathrm{C}(35)-\mathrm{C}(34)-\mathrm{H}(34 \mathrm{~A})$ & 119.7 \\
\hline $\mathrm{C}(33)-\mathrm{C}(34)-\mathrm{H}(34 \mathrm{~A})$ & 119.7 \\
\hline$C(34)-C(35)-C(31)$ & 118. $4(2)$ \\
\hline $\mathrm{C}(34)-\mathrm{C}(35)-\mathrm{H}(35 \mathrm{~A})$ & 120.8 \\
\hline $\mathrm{C}(31)-\mathrm{C}(35)-\mathrm{H}(35 \mathrm{~A})$ & 120.8 \\
\hline$C(37)-C(36)-C(33)$ & $131.4(2)$ \\
\hline$C(37)-C(36)-C(41)$ & $121.1(2)$ \\
\hline$C(33)-C(36)-C(41)$ & $107.52(18)$ \\
\hline$C(38)-C(37)-C(36)$ & $118.7(2)$ \\
\hline $\mathrm{C}(38)-\mathrm{C}(37)-\mathrm{H}(37 \mathrm{~A})$ & 120.6 \\
\hline $\mathrm{C}(36)-\mathrm{C}(37)-\mathrm{H}(37 \mathrm{~A})$ & 120.6 \\
\hline$C(37)-C(38)-C(39)$ & $120.7(2)$ \\
\hline $\mathrm{C}(37)-\mathrm{C}(38)-\mathrm{H}(38 \mathrm{~A})$ & 119.7 \\
\hline $\mathrm{C}(39)-\mathrm{C}(38)-\mathrm{H}(38 \mathrm{~A})$ & 119.7 \\
\hline$C(40)-C(39)-C(38)$ & $122.0(2)$ \\
\hline $\mathrm{C}(40)-\mathrm{C}(39)-\mathrm{H}(39 \mathrm{~A})$ & 119.0 \\
\hline C (38) - C (39)-H (39A) & 119.0 \\
\hline$C(39)-C(40)-C(41)$ & $118.5(2)$ \\
\hline $\mathrm{C}(39)-\mathrm{C}(40)-\mathrm{H}(40 \mathrm{~A})$ & 120.7 \\
\hline $\mathrm{C}(41)-\mathrm{C}(40)-\mathrm{H}(40 \mathrm{~A})$ & 120.7 \\
\hline$C(40)-C(41)-C(42)$ & $133.0(2)$ \\
\hline$C(40)-C(41)-C(36)$ & 118. $97(19)$ \\
\hline$C(42)-C(41)-C(36)$ & $108.04(18)$ \\
\hline$N(3)-C(42)-C(41)$ & 106. $98(17)$ \\
\hline$N(3)-C(42)-C(43)$ & $121.75(19)$ \\
\hline$C(41)-C(42)-C(43)$ & 131. 3(2) \\
\hline $0(5)-C(43)-0(6)$ & $122.6(2)$ \\
\hline $0(5)-C(43)-C(42)$ & $126.5(2)$ \\
\hline $0(6)-C(43)-C(42)$ & $110.85(19)$ \\
\hline $0(6)-C(44)-C(45)$ & $107.3(2)$ \\
\hline $0(6)-C(44)-H(44 A)$ & 110.3 \\
\hline $\mathrm{C}(45)-\mathrm{C}(44)-\mathrm{H}(44 \mathrm{~A})$ & 110.3 \\
\hline $0(6)-\mathrm{C}(44)-\mathrm{H}(44 \mathrm{~B})$ & 110.3 \\
\hline $\mathrm{C}(45)-\mathrm{C}(44)-\mathrm{H}(44 \mathrm{~B})$ & 110.3 \\
\hline $\mathrm{H}(44 \mathrm{~A})-\mathrm{C}(44)-\mathrm{H}(44 \mathrm{~B})$ & 108.5 \\
\hline $\mathrm{C}(44)-\mathrm{C}(45)-\mathrm{H}(45 \mathrm{~A})$ & 109.5 \\
\hline $\mathrm{C}(44)-\mathrm{C}(45)-\mathrm{H}(45 \mathrm{~B})$ & 109.5 \\
\hline
\end{tabular}




$\begin{array}{ll}\mathrm{H}(45 \mathrm{~A})-\mathrm{C}(45)-\mathrm{H}(45 \mathrm{~B}) & 109.5 \\ \mathrm{C}(44)-\mathrm{C}(45)-\mathrm{H}(45 \mathrm{C}) & 109.5 \\ \mathrm{H}(45 \mathrm{~A})-\mathrm{C}(45)-\mathrm{H}(45 \mathrm{C}) & 109.5 \\ \mathrm{H}(45 \mathrm{~B})-\mathrm{C}(45)-\mathrm{H}(45 \mathrm{C}) & 109.5 \\ \mathrm{C}(32)-\mathrm{N}(3)-\mathrm{C}(42) & 129.43(18) \\ \mathrm{C}(32)-\mathrm{N}(3)-\mathrm{C}(33) & 120.41(19) \\ \mathrm{C}(42)-\mathrm{N}(3)-\mathrm{C}(33) & 110.15(17) \\ \mathrm{C}(17)-\mathrm{N}(2)-\mathrm{C}(27) & 129.69(18) \\ \mathrm{C}(17)-\mathrm{N}(2)-\mathrm{C}(18) & 120.70(19) \\ \mathrm{C}(27)-\mathrm{N}(2)-\mathrm{C}(18) & 109.57(18)\end{array}$

Symmetry transformations used to generate equivalent atoms:

Table 11. Anisotropic displacement parameters $\left(A^{\wedge} 2 \times 10^{\wedge} 3\right)$ for $3 \mathrm{~s}$. The anisotropic displacement factor exponent takes the form: $-2 \pi^{2}\left[h^{2} a^{2} U^{11}+\ldots+2 h k a * b * U^{12}\right]$

\begin{tabular}{lcccccr}
\hline & $\mathrm{U} 11$ & $\mathrm{U} 22$ & $\mathrm{U} 33$ & $\mathrm{U} 23$ & $\mathrm{U} 13$ & $\mathrm{U} 12$ \\
$\mathrm{C} 1(1)$ & $53(1)$ & $74(1)$ & $129(1)$ & $2(1)$ & $-14(1)$ & $-11(1)$ \\
$\mathrm{C} 1(2)$ & $57(1)$ & $73(1)$ & $120(1)$ & $-9(1)$ & $-4(1)$ & $5(1)$ \\
$\mathrm{C} 1(3)$ & $56(1)$ & $87(1)$ & $117(1)$ & $10(1)$ & $-8(1)$ & $-16(1)$ \\
$0(1)$ & $53(1)$ & $38(1)$ & $85(1)$ & $-2(1)$ & $2(1)$ & $-2(1)$ \\
$0(2)$ & $48(1)$ & $37(1)$ & $74(1)$ & $-1(1)$ & $2(1)$ & $2(1)$ \\
$0(3)$ & $50(1)$ & $40(1)$ & $71(1)$ & $-1(1)$ & $-2(1)$ & $-4(1)$ \\
$0(4)$ & $55(1)$ & $38(1)$ & $88(1)$ & $3(1)$ & $0(1)$ & $-1(1)$ \\
$0(5)$ & $60(1)$ & $39(1)$ & $76(1)$ & $1(1)$ & $1(1)$ & $-7(1)$ \\
$0(6)$ & $53(1)$ & $38(1)$ & $77(1)$ & $-1(1)$ & $4(1)$ & $-1(1)$ \\
$\mathrm{N}(1)$ & $46(1)$ & $37(1)$ & $45(1)$ & $-2(1)$ & $3(1)$ & $-1(1)$ \\
$\mathrm{C}(1)$ & $51(1)$ & $51(1)$ & $60(2)$ & $-2(1)$ & $-3(1)$ & $-2(1)$ \\
$\mathrm{C}(2)$ & $53(1)$ & $42(1)$ & $58(1)$ & $-2(1)$ & $2(1)$ & $-3(1)$ \\
$\mathrm{C}(3)$ & $56(1)$ & $37(1)$ & $43(1)$ & $0(1)$ & $5(1)$ & $3(1)$ \\
$\mathrm{C}(4)$ & $59(1)$ & $43(1)$ & $56(1)$ & $2(1)$ & $2(1)$ & $7(1)$ \\
$\mathrm{C}(5)$ & $53(1)$ & $55(2)$ & $63(2)$ & $1(1)$ & $-1(1)$ & $10(1)$ \\
$\mathrm{C}(6)$ & $55(1)$ & $39(1)$ & $43(1)$ & $0(1)$ & $6(1)$ & $-2(1)$ \\
$\mathrm{C}(7)$ & $68(2)$ & $39(1)$ & $62(2)$ & $4(1)$ & $4(1)$ & $-4(1)$ \\
$\mathrm{C}(8)$ & $75(2)$ & $45(1)$ & $76(2)$ & $4(1)$ & $6(1)$ & $-17(1)$ \\
$\mathrm{C}(9)$ & $56(1)$ & $60(2)$ & $74(2)$ & $4(1)$ & $6(1)$ & $-16(1)$ \\
$\mathrm{C}(10)$ & $52(1)$ & $49(1)$ & $64(2)$ & $1(1)$ & $6(1)$ & $-2(1)$
\end{tabular}




\begin{tabular}{|c|c|c|c|c|c|c|}
\hline$C(11)$ & $52(1)$ & $38(1)$ & $45(1)$ & $-1(1)$ & $6(1)$ & $-1(1)$ \\
\hline$C(12)$ & $46(1)$ & $38(1)$ & $48(1)$ & $0(1)$ & $3(1)$ & $0(1)$ \\
\hline$C(13)$ & $48(1)$ & $39(1)$ & $47(1)$ & $-2(1)$ & $3(1)$ & $-1(1)$ \\
\hline C (14) & $56(1)$ & $38(1)$ & $61(1)$ & $0(1)$ & $6(1)$ & $7(1)$ \\
\hline$C(15)$ & $57(2)$ & $63(2)$ & $125(3)$ & $3(2)$ & $7(2)$ & $13(1)$ \\
\hline$C(16)$ & $56(1)$ & $56(2)$ & $63(2)$ & $-3(1)$ & $1(1)$ & $-1(1)$ \\
\hline$C(17)$ & $58(1)$ & $43(1)$ & $56(1)$ & $-1(1)$ & $0(1)$ & $-1(1)$ \\
\hline$C(18)$ & $64(1)$ & $37(1)$ & $42(1)$ & $-1(1)$ & $4(1)$ & $-5(1)$ \\
\hline C (19) & $68(2)$ & $43(1)$ & $62(2)$ & $-5(1)$ & $5(1)$ & $-12(1)$ \\
\hline$C(20)$ & $58(2)$ & $54(2)$ & $78(2)$ & $-7(1)$ & $-2(1)$ & $-12(1)$ \\
\hline$C(21)$ & $62(1)$ & $41(1)$ & $40(1)$ & $0(1)$ & $5(1)$ & $-2(1)$ \\
\hline$C(22)$ & $79(2)$ & $41(1)$ & $55(1)$ & $-3(1)$ & $9(1)$ & 1(1) \\
\hline$C(23)$ & $82(2)$ & $50(2)$ & $70(2)$ & $-1(1)$ & $8(1)$ & $17(1)$ \\
\hline$C(24)$ & $65(2)$ & $61(2)$ & $66(2)$ & $-4(1)$ & $5(1)$ & $13(1)$ \\
\hline$C(25)$ & $58(1)$ & $54(2)$ & $56(1)$ & $-5(1)$ & $5(1)$ & $-1(1)$ \\
\hline$C(26)$ & $60(1)$ & $40(1)$ & $41(1)$ & $-2(1)$ & $5(1)$ & $-1(1)$ \\
\hline$C(27)$ & $50(1)$ & $40(1)$ & $49(1)$ & $-2(1)$ & $4(1)$ & $-5(1)$ \\
\hline C (28) & $53(1)$ & $42(1)$ & $45(1)$ & 1(1) & $1(1)$ & $-4(1)$ \\
\hline C (29) & $53(1)$ & $44(1)$ & $58(1)$ & $-2(1)$ & $4(1)$ & $-10(1)$ \\
\hline$C(30)$ & $56(2)$ & $72(2)$ & $95(2)$ & $10(2)$ & $-2(1)$ & $-14(1)$ \\
\hline C (31) & $51(1)$ & $64(2)$ & $58(2)$ & $4(1)$ & $-1(1)$ & $-4(1)$ \\
\hline$C(32)$ & $54(1)$ & $46(1)$ & $55(1)$ & 2(1) & $2(1)$ & $-8(1)$ \\
\hline C (33) & $59(1)$ & $38(1)$ & $43(1)$ & $-1(1)$ & $5(1)$ & $0(1)$ \\
\hline C (34) & $65(2)$ & $50(1)$ & $63(2)$ & $-1(1)$ & $2(1)$ & $8(1)$ \\
\hline C (35) & $56(2)$ & $65(2)$ & $76(2)$ & $0(1)$ & $-1(1)$ & $9(1)$ \\
\hline$C(36)$ & $57(1)$ & $40(1)$ & $41(1)$ & $-2(1)$ & $7(1)$ & $-5(1)$ \\
\hline C (37) & $74(2)$ & $37(1)$ & $59(1)$ & $-1(1)$ & $7(1)$ & $-4(1)$ \\
\hline C (38) & $77(2)$ & $42(1)$ & $75(2)$ & $-3(1)$ & $9(1)$ & $-16(1)$ \\
\hline C (39) & $61(2)$ & $56(2)$ & $76(2)$ & $-4(1)$ & $10(1)$ & $-17(1)$ \\
\hline$C(40)$ & $56(1)$ & $48(1)$ & $64(2)$ & $-3(1)$ & $9(1)$ & $-5(1)$ \\
\hline$C(41)$ & $56(1)$ & $38(1)$ & $43(1)$ & $-2(1)$ & $7(1)$ & $-5(1)$ \\
\hline$C(42)$ & $48(1)$ & $38(1)$ & $50(1)$ & $-3(1)$ & $5(1)$ & $-1(1)$ \\
\hline C (43) & $53(1)$ & $42(1)$ & $45(1)$ & $-1(1)$ & $5(1)$ & $-4(1)$ \\
\hline$C(44)$ & $64(1)$ & $39(1)$ & $65(2)$ & $-1(1)$ & $5(1)$ & $5(1)$ \\
\hline$C(45)$ & $64(2)$ & $62(2)$ & $139(3)$ & $13(2)$ & $11(2)$ & $13(1)$ \\
\hline $\mathrm{N}(3)$ & $51(1)$ & $38(1)$ & $43(1)$ & $0(1)$ & $4(1)$ & $-4(1)$ \\
\hline $\mathrm{N}(2)$ & $52(1)$ & $40(1)$ & $46(1)$ & $-1(1)$ & $2(1)$ & $-4(1)$ \\
\hline
\end{tabular}


Table 12. Hydrogen coordinates $\left(\times 10^{\wedge} 4\right)$ and isotropic displacement parameters $\left(A^{\wedge} 2 \times 10^{\wedge} 3\right)$ for 3s.

\begin{tabular}{|c|c|c|c|c|}
\hline & $\mathrm{x}$ & $\mathrm{y}$ & z & $\mathrm{U}(\mathrm{eq})$ \\
\hline $\mathrm{H}(2 \mathrm{~A})$ & 9021 & 3555 & 2494 & 61 \\
\hline $\mathrm{H}(4 \mathrm{~A})$ & 8942 & 6343 & 2382 & 64 \\
\hline $\mathrm{H}(5 \mathrm{~A})$ & 7220 & 5619 & 2277 & 68 \\
\hline $\mathrm{H}(7 \mathrm{~A})$ & 11171 & 6879 & 2534 & 68 \\
\hline H(8A) & 13168 & 7029 & 2664 & 78 \\
\hline H(9A) & 14370 & 5910 & 2777 & 76 \\
\hline $\mathrm{H}(10 \mathrm{~A})$ & 13631 & 4628 & 2778 & 66 \\
\hline $\mathrm{H}(14 \mathrm{~A})$ & 12739 & 2214 & 2342 & 62 \\
\hline $\mathrm{H}(14 \mathrm{~B})$ & 12666 & 2198 & 3104 & 62 \\
\hline $\mathrm{H}(15 \mathrm{~A})$ & 14566 & 1926 & 2846 & 122 \\
\hline $\mathrm{H}(15 \mathrm{~B})$ & 14538 & 2735 & 3238 & 122 \\
\hline $\mathrm{H}(15 \mathrm{C})$ & 14612 & 2752 & 2479 & 122 \\
\hline $\mathrm{H}(17 \mathrm{~A})$ & 8753 & 2945 & 852 & 63 \\
\hline $\mathrm{H}(19 \mathrm{~A})$ & 8645 & 162 & 685 & 69 \\
\hline $\mathrm{H}(20 \mathrm{~A})$ & 6940 & 888 & 604 & 76 \\
\hline $\mathrm{H}(22 \mathrm{~A})$ & 10890 & -388 & 851 & 70 \\
\hline $\mathrm{H}(23 \mathrm{~A})$ & 12877 & -540 & 1035 & 80 \\
\hline $\mathrm{H}(24 \mathrm{~A})$ & 14078 & 567 & 1186 & 77 \\
\hline $\mathrm{H}(25 \mathrm{~A})$ & 13356 & 1857 & 1136 & 67 \\
\hline $\mathrm{H}(29 \mathrm{~A})$ & 12386 & 4303 & 1425 & 62 \\
\hline $\mathrm{H}(29 \mathrm{~B})$ & 12564 & 4249 & 673 & 62 \\
\hline $\mathrm{H}(30 \mathrm{~A})$ & 14347 & 4516 & 1245 & 112 \\
\hline $\mathrm{H}(30 \mathrm{~B})$ & 14398 & 3672 & 905 & 112 \\
\hline $\mathrm{H}(30 \mathrm{C})$ & 14221 & 3726 & 1654 & 112 \\
\hline $\mathrm{H}(32 \mathrm{~A})$ & 10684 & 7925 & 860 & 63 \\
\hline $\mathrm{H}(34 \mathrm{~A})$ & 10802 & 5140 & 806 & 71 \\
\hline $\mathrm{H}(35 \mathrm{~A})$ & 12507 & 5862 & 933 & 79 \\
\hline $\mathrm{H}(37 \mathrm{~A})$ & 8538 & 4595 & 682 & 68 \\
\hline $\mathrm{H}(38 \mathrm{~A})$ & 6543 & 4453 & 584 & 78 \\
\hline $\mathrm{H}(39 \mathrm{~A})$ & 5341 & 5572 & 535 & 77 \\
\hline
\end{tabular}




$\begin{array}{llrrr}\text { H(40A) } & 6092 & 6857 & 560 & 67 \\ \text { H(44A) } & 7028 & 9279 & 266 & 67 \\ \text { H(44B) } & 6962 & 9259 & 1029 & 67 \\ \text { H(45A) } & 5119 & 9524 & 530 & 132 \\ \text { H(45B) } & 5102 & 8695 & 896 & 132 \\ \text { H(45C) } & 5168 & 8717 & 137 & 132\end{array}$

Table 13. Torsion angles [deg] for sad.

\begin{tabular}{lc}
\hline $\mathrm{C}(5)-\mathrm{C}(1)-\mathrm{C}(2)-\mathrm{N}(1)$ & $-0.1(4)$ \\
$\mathrm{C} 1(1)-\mathrm{C}(1)-\mathrm{C}(2)-\mathrm{N}(1)$ & $-178.91(17)$ \\
$\mathrm{C}(12)-\mathrm{N}(1)-\mathrm{C}(2)-\mathrm{C}(1)$ & $-179.2(2)$ \\
$\mathrm{C}(3)-\mathrm{N}(1)-\mathrm{C}(2)-\mathrm{C}(1)$ & $0.2(3)$ \\
$\mathrm{C}(2)-\mathrm{N}(1)-\mathrm{C}(3)-\mathrm{C}(4)$ & $-0.2(3)$ \\
$\mathrm{C}(12)-\mathrm{N}(1)-\mathrm{C}(3)-\mathrm{C}(4)$ & $179.3(2)$ \\
$\mathrm{C}(2)-\mathrm{N}(1)-\mathrm{C}(3)-\mathrm{C}(6)$ & $179.98(19)$ \\
$\mathrm{C}(12)-\mathrm{N}(1)-\mathrm{C}(3)-\mathrm{C}(6)$ & $-0.5(2)$ \\
$\mathrm{N}(1)-\mathrm{C}(3)-\mathrm{C}(4)-\mathrm{C}(5)$ & $0.1(3)$ \\
$\mathrm{C}(6)-\mathrm{C}(3)-\mathrm{C}(4)-\mathrm{C}(5)$ & $179.8(2)$ \\
$\mathrm{C}(3)-\mathrm{C}(4)-\mathrm{C}(5)-\mathrm{C}(1)$ & $0.1(4)$ \\
$\mathrm{C}(2)-\mathrm{C}(1)-\mathrm{C}(5)-\mathrm{C}(4)$ & $-0.1(4)$ \\
$\mathrm{C} 1(1)-\mathrm{C}(1)-\mathrm{C}(5)-\mathrm{C}(4)$ & $178.74(18)$ \\
$\mathrm{C}(4)-\mathrm{C}(3)-\mathrm{C}(6)-\mathrm{C}(7)$ & $0.0(4)$ \\
$\mathrm{N}(1)-\mathrm{C}(3)-\mathrm{C}(6)-\mathrm{C}(7)$ & $179.7(2)$ \\
$\mathrm{C}(4)-\mathrm{C}(3)-\mathrm{C}(6)-\mathrm{C}(11)$ & $-179.3(2)$ \\
$\mathrm{N}(1)-\mathrm{C}(3)-\mathrm{C}(6)-\mathrm{C}(11)$ & $0.5(2)$ \\
$\mathrm{C}(3)-\mathrm{C}(6)-\mathrm{C}(7)-\mathrm{C}(8)$ & $-179.0(2)$ \\
$\mathrm{C}(11)-\mathrm{C}(6)-\mathrm{C}(7)-\mathrm{C}(8)$ & $0.1(3)$ \\
$\mathrm{C}(6)-\mathrm{C}(7)-\mathrm{C}(8)-\mathrm{C}(9)$ & $0.2(4)$ \\
$\mathrm{C}(7)-\mathrm{C}(8)-\mathrm{C}(9)-\mathrm{C}(10)$ & $-0.4(4)$ \\
$\mathrm{C}(8)-\mathrm{C}(9)-\mathrm{C}(10)-\mathrm{C}(11)$ & $0.3(4)$ \\
$\mathrm{C}(9)-\mathrm{C}(10)-\mathrm{C}(11)-\mathrm{C}(12)$ & $-0.2(3)$ \\
$\mathrm{C}(9)-\mathrm{C}(10)-\mathrm{C}(11)-\mathrm{C}(6)$ & $179.1(2)$ \\
$\mathrm{C}(7)-\mathrm{C}(6)-\mathrm{C}(11)-\mathrm{C}(10)$ & $-179.6(2)$ \\
$\mathrm{C}(3)-\mathrm{C}(6)-\mathrm{C}(11)-\mathrm{C}(10)$ & \\
$\mathrm{C}(7)-\mathrm{C}(6)-\mathrm{C}(11)-\mathrm{C}(12)$ & \\
$\mathrm{C}(3)-\mathrm{C}(6)-\mathrm{C}(11)-\mathrm{C}(12)$ & \\
& \\
&
\end{tabular}




\begin{tabular}{|c|c|}
\hline $\mathrm{C}(2)-\mathrm{N}(1)-\mathrm{C}(12)-\mathrm{C}(11)$ & $179.8(2)$ \\
\hline $\mathrm{C}(3)-\mathrm{N}(1)-\mathrm{C}(12)-\mathrm{C}(11)$ & $0.3(2)$ \\
\hline $\mathrm{C}(2)-\mathrm{N}(1)-\mathrm{C}(12)-\mathrm{C}(13)$ & 1. $0(3)$ \\
\hline $\mathrm{C}(3)-\mathrm{N}(1)-\mathrm{C}(12)-\mathrm{C}(13)$ & $-178.49(19)$ \\
\hline $\mathrm{C}(10)-\mathrm{C}(11)-\mathrm{C}(12)-\mathrm{N}(1)$ & $-179.3(2)$ \\
\hline $\mathrm{C}(6)-\mathrm{C}(11)-\mathrm{C}(12)-\mathrm{N}(1)$ & $0.0(2)$ \\
\hline$C(10)-C(11)-C(12)-C(13)$ & $-0.6(4)$ \\
\hline$C(6)-C(11)-C(12)-C(13)$ & $178.6(2)$ \\
\hline$C(14)-0(2)-C(13)-0(1)$ & 2. $2(3)$ \\
\hline$C(14)-0(2)-C(13)-C(12)$ & $-177.65(18)$ \\
\hline $\mathrm{N}(1)-\mathrm{C}(12)-\mathrm{C}(13)-0(1)$ & $-1.2(3)$ \\
\hline$C(11)-C(12)-C(13)-0(1)$ & $-179.6(2)$ \\
\hline$N(1)-C(12)-C(13)-0(2)$ & 178. $72(18)$ \\
\hline$C(11)-C(12)-C(13)-0(2)$ & $0.3(3)$ \\
\hline$C(13)-0(2)-C(14)-C(15)$ & $-179.8(2)$ \\
\hline $\mathrm{C}(20)-\mathrm{C}(16)-\mathrm{C}(17)-\mathrm{N}(2)$ & $-0.8(4)$ \\
\hline $\mathrm{C} 1(2)-\mathrm{C}(16)-\mathrm{C}(17)-\mathrm{N}(2)$ & $177.68(17)$ \\
\hline$N(2)-C(18)-C(19)-C(20)$ & $-0.7(3)$ \\
\hline$C(21)-C(18)-C(19)-C(20)$ & 177. $3(2)$ \\
\hline$C(18)-C(19)-C(20)-C(16)$ & $-1.0(4)$ \\
\hline$C(17)-C(16)-C(20)-C(19)$ & $1.9(4)$ \\
\hline $\mathrm{Cl}(2)-\mathrm{C}(16)-\mathrm{C}(20)-\mathrm{C}(19)$ & $-176.6(2)$ \\
\hline$C(19)-C(18)-C(21)-C(22)$ & $0.5(4)$ \\
\hline$N(2)-C(18)-C(21)-C(22)$ & $178.7(2)$ \\
\hline$C(19)-C(18)-C(21)-C(26)$ & $-178.9(2)$ \\
\hline$N(2)-C(18)-C(21)-C(26)$ & $-0.7(2)$ \\
\hline$C(18)-C(21)-C(22)-C(23)$ & $-178.5(2)$ \\
\hline$C(26)-C(21)-C(22)-C(23)$ & $0.8(3)$ \\
\hline$C(21)-C(22)-C(23)-C(24)$ & $-0.4(4)$ \\
\hline$C(22)-C(23)-C(24)-C(25)$ & $-0.7(4)$ \\
\hline$C(23)-C(24)-C(25)-C(26)$ & 1. $4(4)$ \\
\hline$C(24)-C(25)-C(26)-C(27)$ & 178. 4(2) \\
\hline$C(24)-C(25)-C(26)-C(21)$ & $-0.9(3)$ \\
\hline$C(22)-C(21)-C(26)-C(27)$ & $-179.7(2)$ \\
\hline$C(18)-C(21)-C(26)-C(27)$ & $-0.2(2)$ \\
\hline$C(22)-C(21)-C(26)-C(25)$ & $-0.2(3)$ \\
\hline$C(18)-C(21)-C(26)-C(25)$ & 179. $30(19)$ \\
\hline $\mathrm{C}(25)-\mathrm{C}(26)-\mathrm{C}(27)-\mathrm{N}(2)$ & $-178.3(2)$ \\
\hline
\end{tabular}




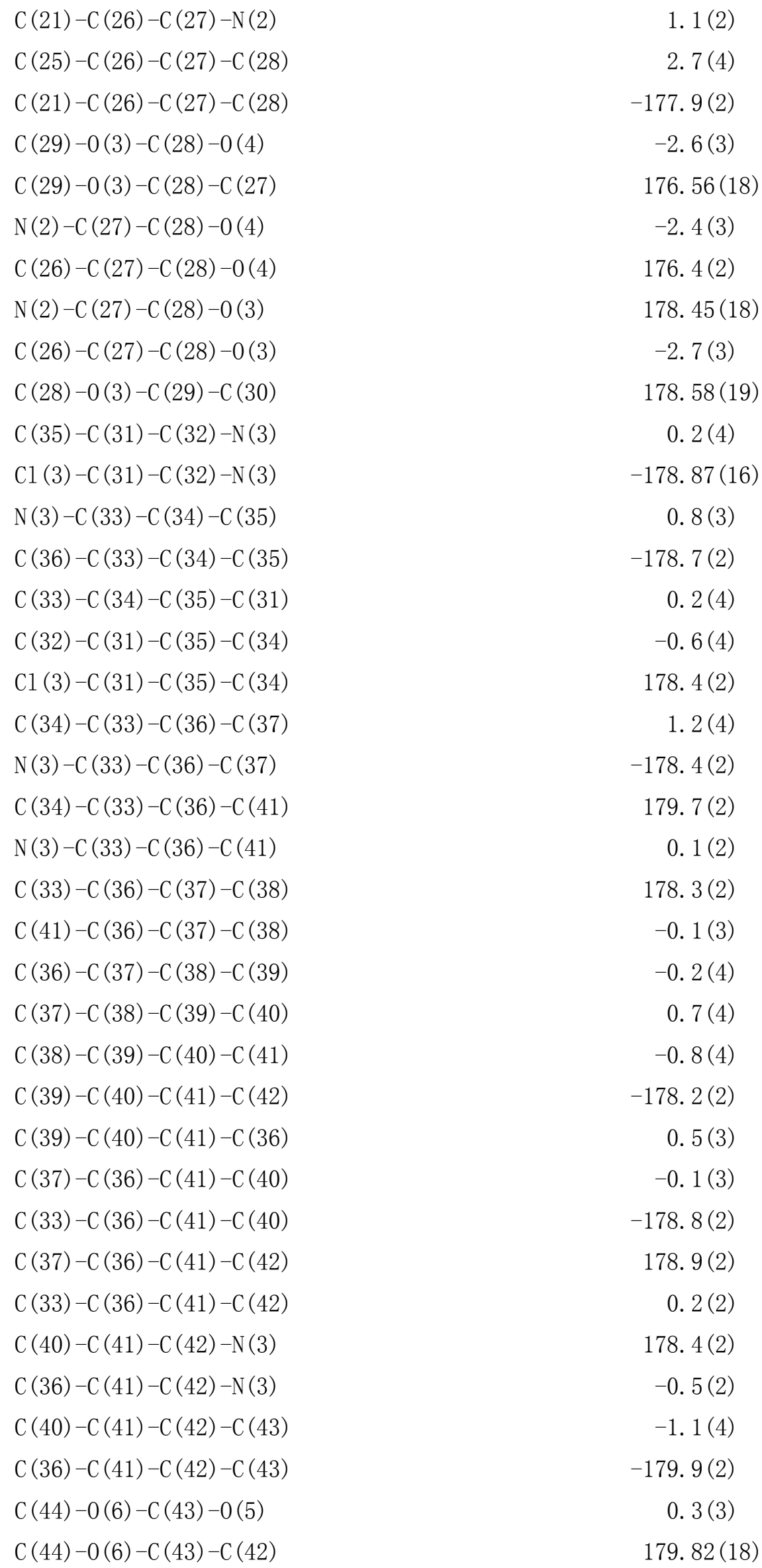




$$
\begin{aligned}
& N(3)-C(42)-C(43)-0(5) \\
& 0.9(3) \\
& \text { C (41) }-\mathrm{C}(42)-\mathrm{C}(43)-0(5) \\
& -179.6(2) \\
& \mathrm{N}(3)-\mathrm{C}(42)-\mathrm{C}(43)-0 \text { (6) } \\
& -178.57(18) \\
& \text { C (41) }-\mathrm{C}(42)-\mathrm{C}(43)-0 \text { (6) } \\
& 0.8(3) \\
& \mathrm{C}(43)-0(6)-\mathrm{C}(44)-\mathrm{C}(45) \\
& \text { 178. } 3(2) \\
& \mathrm{C}(31)-\mathrm{C}(32)-\mathrm{N}(3)-\mathrm{C}(42) \\
& \text { 179. } 4(2) \\
& \mathrm{C}(31)-\mathrm{C}(32)-\mathrm{N}(3)-\mathrm{C}(33) \\
& 0.8(3) \\
& \mathrm{C}(41)-\mathrm{C}(42)-\mathrm{N}(3)-\mathrm{C}(32) \\
& \mathrm{C}(43)-\mathrm{C}(42)-\mathrm{N}(3)-\mathrm{C}(32) \\
& -178.1(2) \\
& \mathrm{C}(41)-\mathrm{C}(42)-\mathrm{N}(3)-\mathrm{C}(33) \\
& \text { 1. } 4(3) \\
& \mathrm{C}(43)-\mathrm{C}(42)-\mathrm{N}(3)-\mathrm{C}(33) \\
& 0.6(2) \\
& \mathrm{C}(34)-\mathrm{C}(33)-\mathrm{N}(3)-\mathrm{C}(32) \\
& -179.90(19) \\
& \mathrm{C}(36)-\mathrm{C}(33)-\mathrm{N}(3)-\mathrm{C}(32) \\
& -1.3(3) \\
& \mathrm{C}(34)-\mathrm{C}(33)-\mathrm{N}(3)-\mathrm{C}(42) \\
& \text { 178. } 36(18) \\
& \mathrm{C}(36)-\mathrm{C}(33)-\mathrm{N}(3)-\mathrm{C}(42) \\
& \mathrm{C}(16)-\mathrm{C}(17)-\mathrm{N}(2)-\mathrm{C}(27) \\
& \mathrm{C}(16)-\mathrm{C}(17)-\mathrm{N}(2)-\mathrm{C}(18) \\
& \mathrm{C}(26)-\mathrm{C}(27)-\mathrm{N}(2)-\mathrm{C}(17) \\
& \text { 179. } 9(2) \\
& -0.4(2) \\
& -178.8(2) \\
& -1.0(3) \\
& \mathrm{C}(28)-\mathrm{C}(27)-\mathrm{N}(2)-\mathrm{C}(17) \\
& \text { 176. } 4(2) \\
& \mathrm{C}(26)-\mathrm{C}(27)-\mathrm{N}(2)-\mathrm{C}(18) \\
& -4.5(3) \\
& \mathrm{C}(28)-\mathrm{C}(27)-\mathrm{N}(2)-\mathrm{C}(18) \\
& -1.6(2) \\
& \mathrm{C}(19)-\mathrm{C}(18)-\mathrm{N}(2)-\mathrm{C}(17) \\
& \text { 177. } 51(19) \\
& \mathrm{C}(21)-\mathrm{C}(18)-\mathrm{N}(2)-\mathrm{C}(17) \\
& \text { 1. } 8(3) \\
& \mathrm{C}(19)-\mathrm{C}(18)-\mathrm{N}(2)-\mathrm{C}(27) \\
& -176.73(18) \\
& \mathrm{C}(21)-\mathrm{C}(18)-\mathrm{N}(2)-\mathrm{C}(27)
\end{aligned}
$$

\section{References}

1. Sun, B.; Yoshino, T.; Matsunaga, S.; Kanai, M. Adv. Synth. Catal. 2014, 356, 1491.

2. Yu, D. G.; Gensch, T.; Azambuja, F. d.; Céspedes, S. V.; Glorius, F. J. Am. Chem. Soc. 2014, 136,17722 .

3. (a) Chen, X.; Goodhue, C. E.; Yu, J. -Q. J. Am. Chem. Soc. 2006, 128, 12634; (b) Mizuno, H.; Takaya, J.; Iwasawa, N. J. Am. Chem. Soc. 2011, 133, 1251.

4. (a) Waser, J.; Gaspar, B.; Nambu, H.; Carreira, E. M. J. Am. Chem. Soc. 2006, 128, 11693; (b) Itoh, M.; Hirano, K.; Satoh, T.; Miura, M. Org. Lett. 2014, 16, 2050; (c) Chaitanya, M.; Anbarasan, P. Org. Lett. 2015, 17, 3766.

5. Kim, C.; Baek, Y.; Kim, S. H.; Lee, P. H. Adv. Synth. Catal. 2015, 357, 2903. 
6. Huang, X.; Zhang, T. Tetrahedron Lett. 2009, 50, 208.

7. Yang, L.; Correia, C. A.; Li, C. -J. Adv. Synth. Catal. 2011, 353, 1269. 
5. ${ }^{1} \mathrm{H}$ NMR and ${ }^{13} \mathrm{C}$ NMR spectrum for all isolated products.

(1) The ${ }^{1} \mathrm{H}$ NMR and ${ }^{13} \mathrm{C}$ NMR spectrum for $\mathbf{3 a}$ (using $\mathrm{CDCl}_{3}$ as solvent)
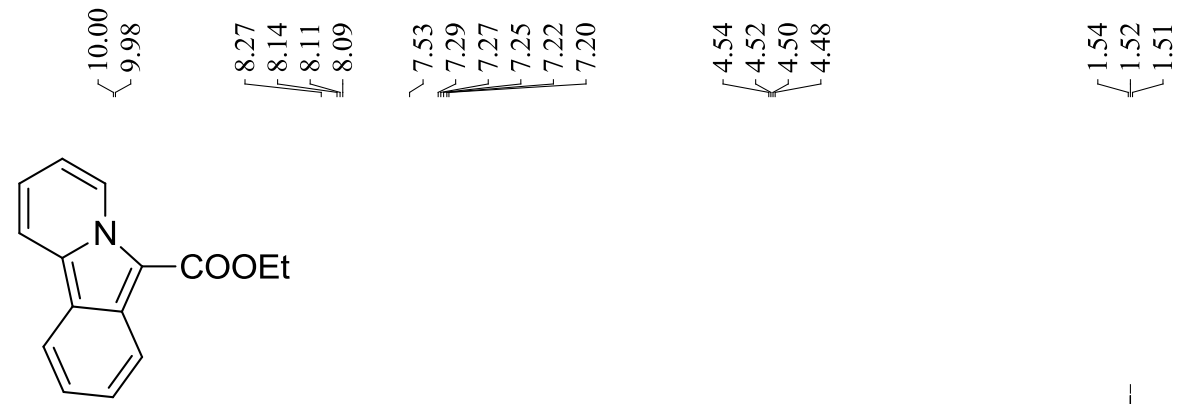

$3 a$

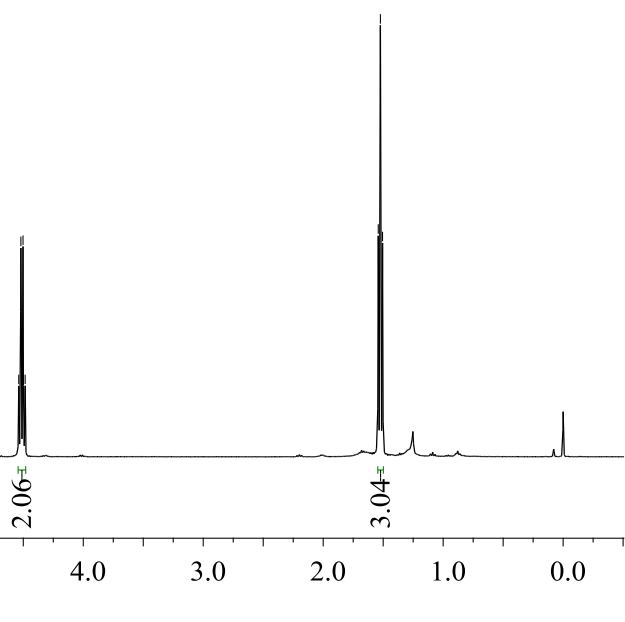

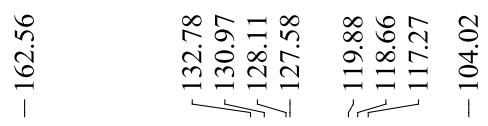

i⿱n

$\stackrel{\infty}{\infty}$<smiles>CCOC(=O)c1c2ccccc2c2ccccn12</smiles>

$3 a$ 
(2) The ${ }^{1} \mathrm{H}$ NMR and ${ }^{13} \mathrm{C}$ NMR spectrum for $\mathbf{3 b}$ (using $\mathrm{CDCl}_{3}$ as solvent)

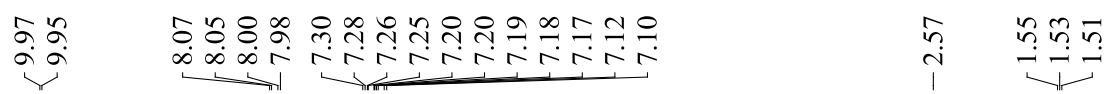

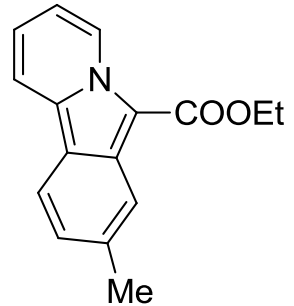

$3 b$

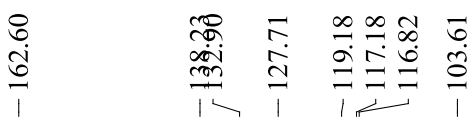

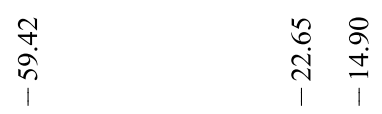<smiles>CCOC(=O)c1c2cc(C)ccc2c2ccccn12</smiles>

$3 b$

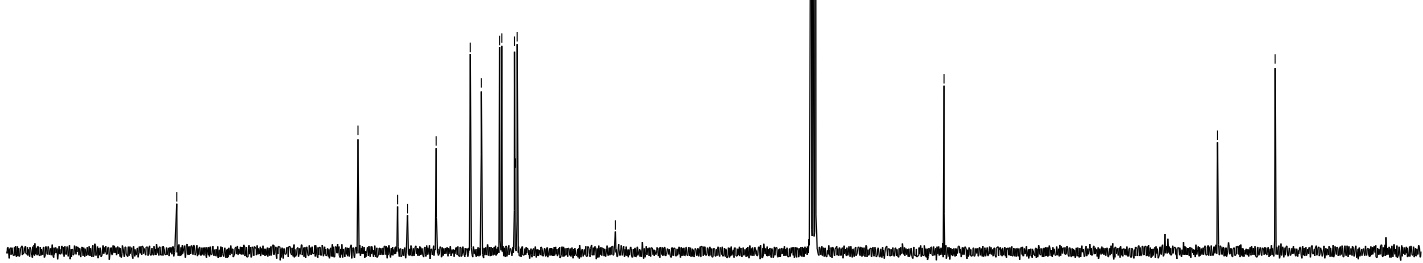

$\begin{array}{lllllllllllllllllll}180 & 170 & 160 & 150 & 140 & 130 & 120 & 110 & 100 & 90 & 80 & 70 & 60 & 50 & 40 & 30 & 20 & 10 & 0\end{array}$ 
(3) The ${ }^{1} \mathrm{H}$ NMR and ${ }^{13} \mathrm{C}$ NMR spectrum for $3 \mathbf{c}$ (using $\mathrm{CDCl}_{3}$ as solvent)

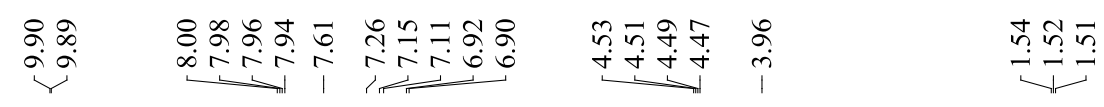<smiles>CCOC(=O)c1c2cc(OC)ccc2c2ccccn12</smiles>

3c
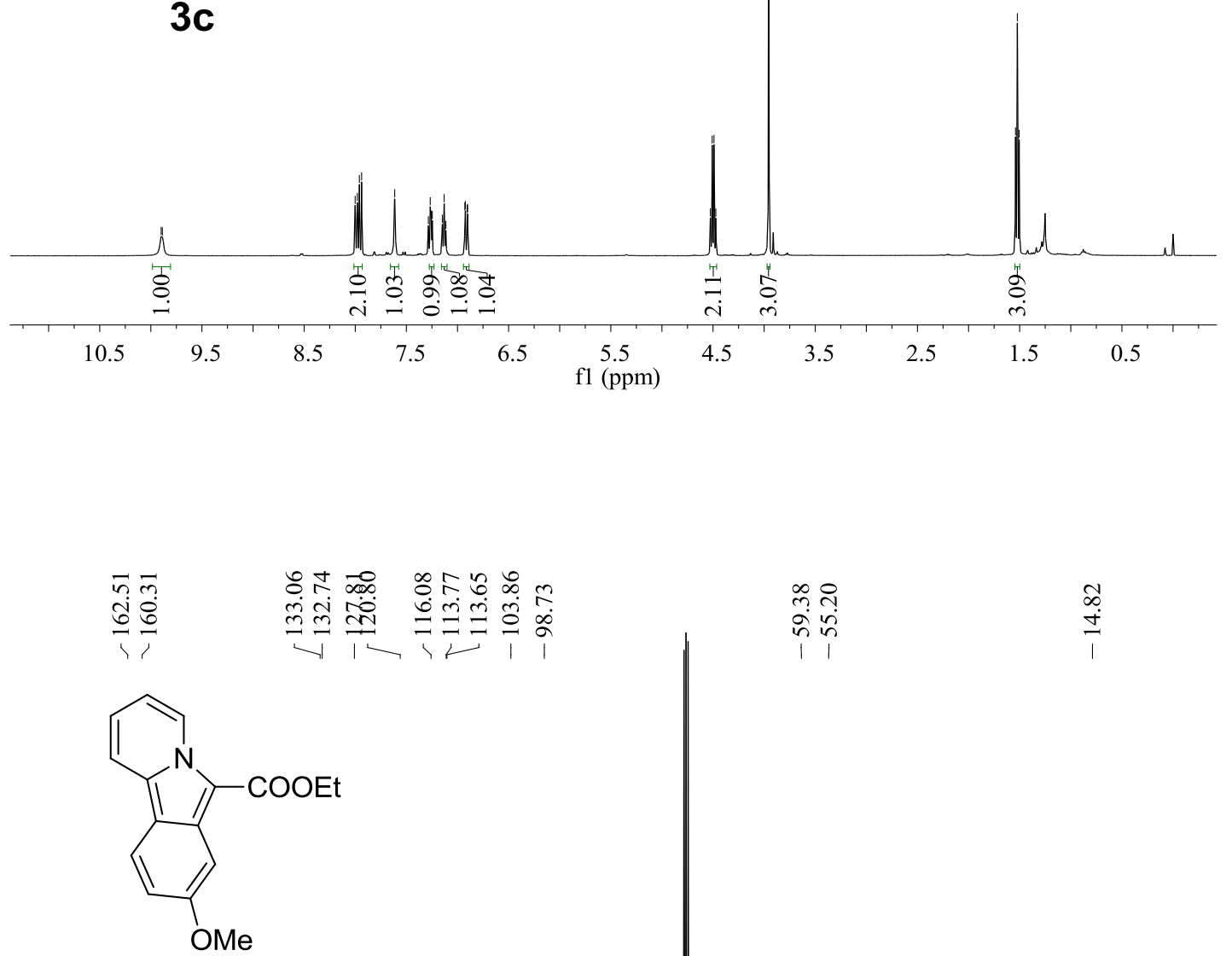

3c
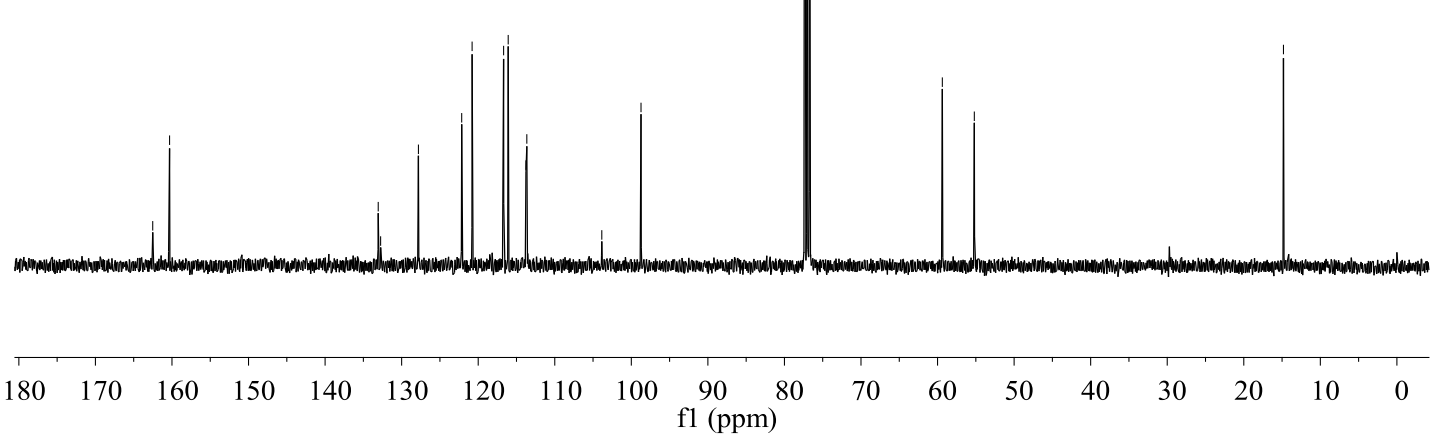
(4) The ${ }^{1} \mathrm{H}$ NMR and ${ }^{13} \mathrm{C}$ NMR spectrum for $\mathbf{3 d}$ (using $\mathrm{CDCl}_{3}$ as solvent)
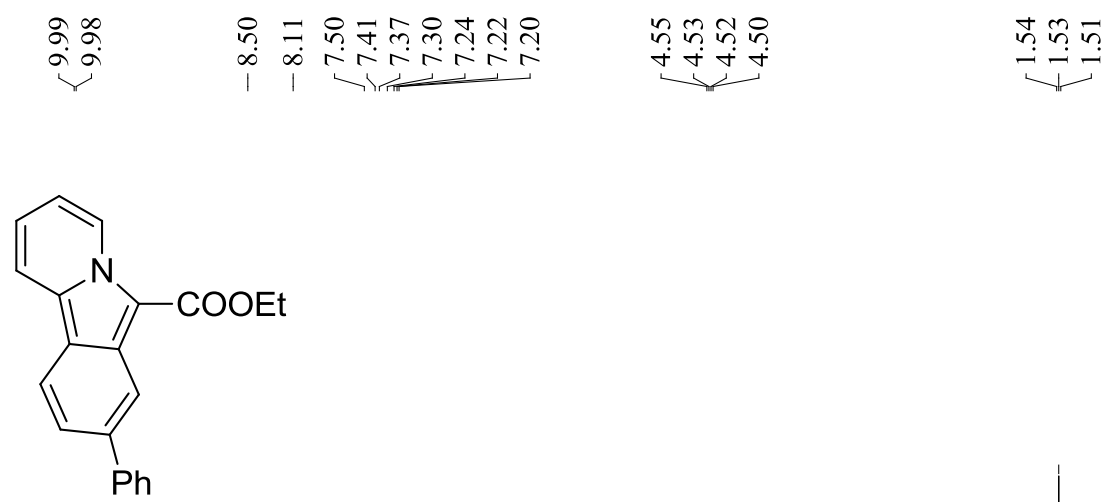

3d

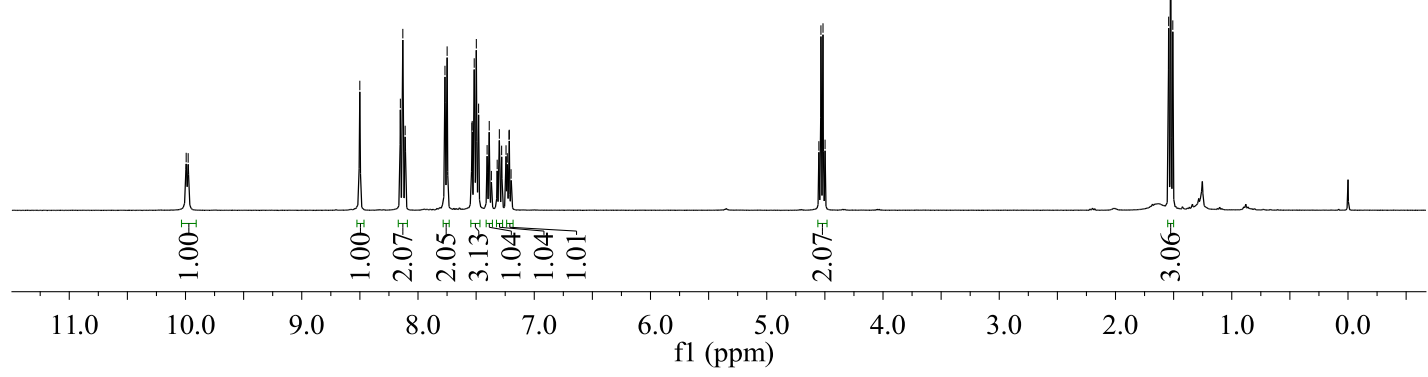

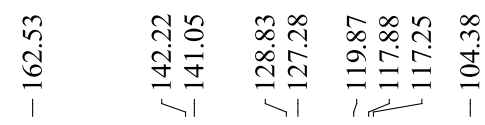
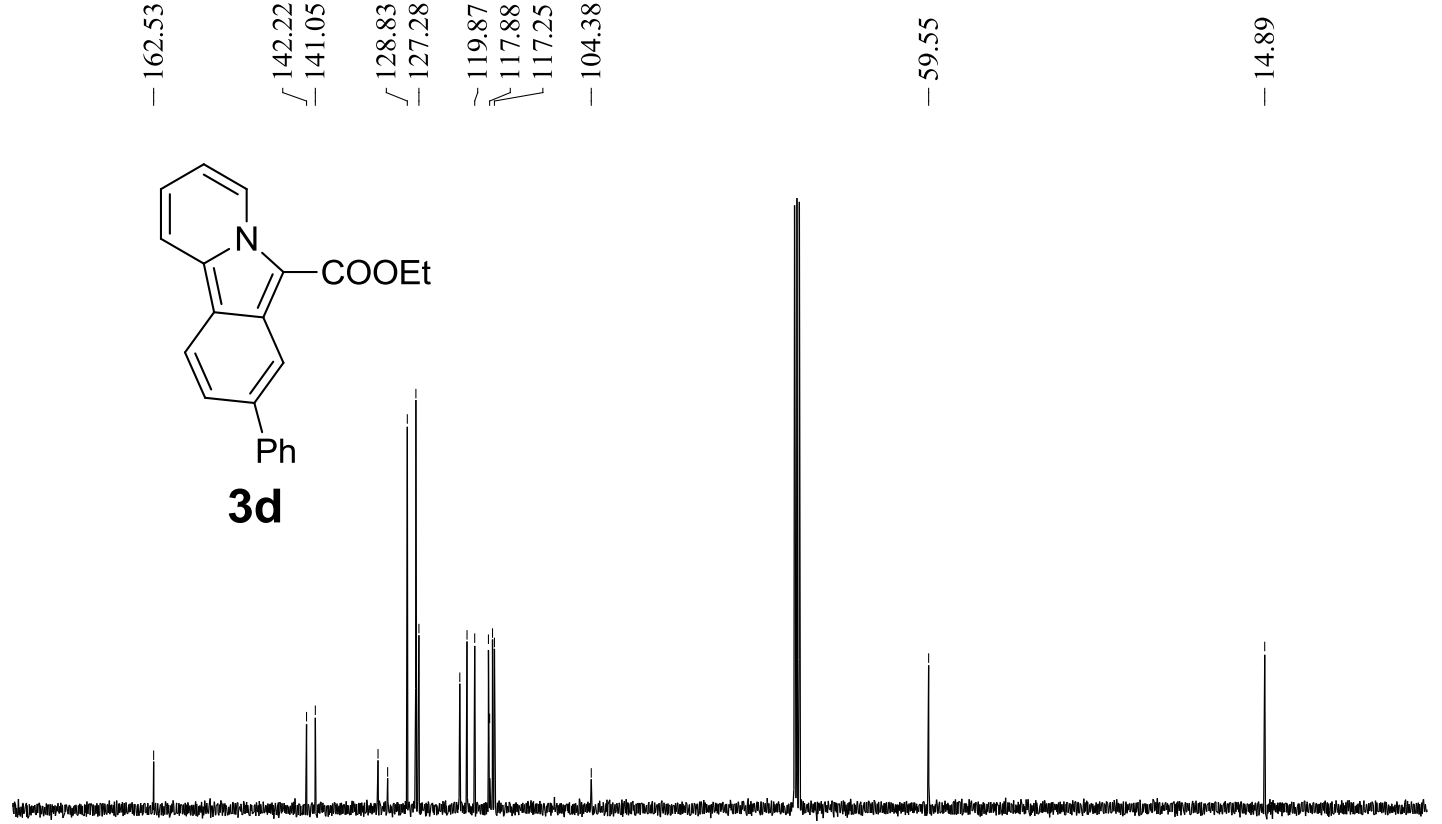

$\stackrel{+}{\dot{1}}$ 
(5) The ${ }^{1} \mathrm{H}$ NMR and ${ }^{13} \mathrm{C}$ NMR spectrum for $3 e$ (using $\mathrm{CDCl}_{3}$ as solvent)

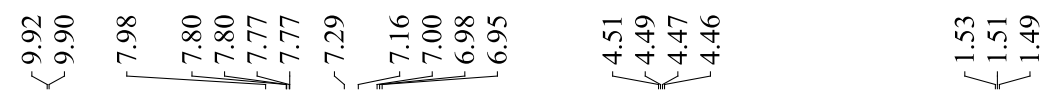<smiles>CCOC(=O)c1c2cc(F)ccc2c2ccccn12</smiles>

$3 e$

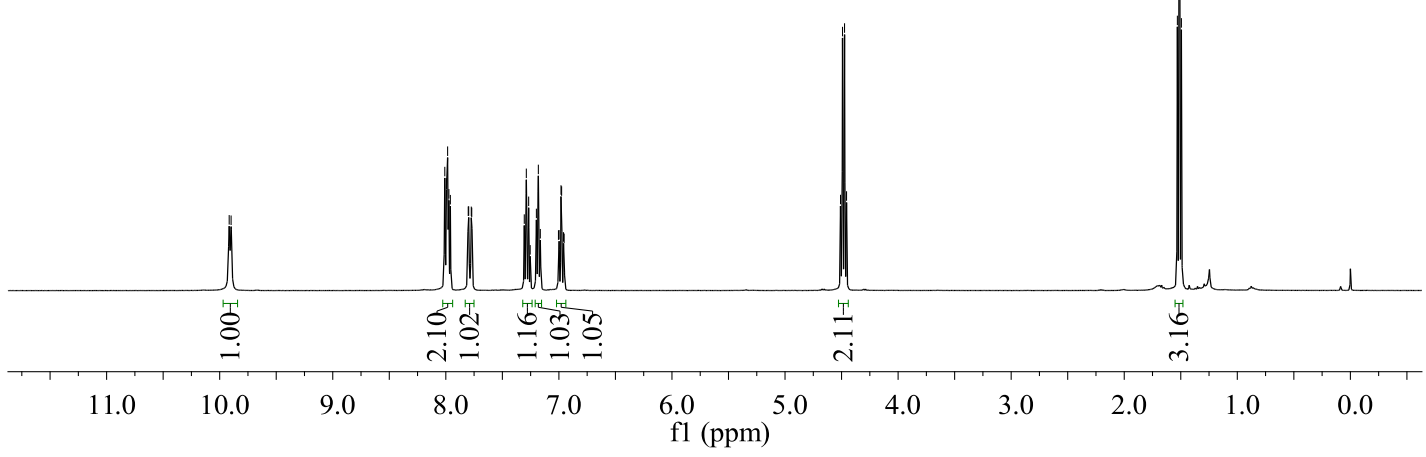

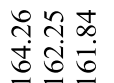

은

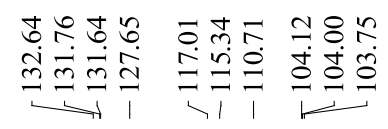

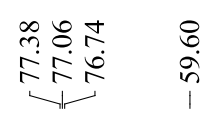

$\stackrel{\substack{\infty \\ \dot{ \pm}}}{1}$<smiles>CCOC(=O)c1c2cc(F)ccc2c2ccccn12</smiles>

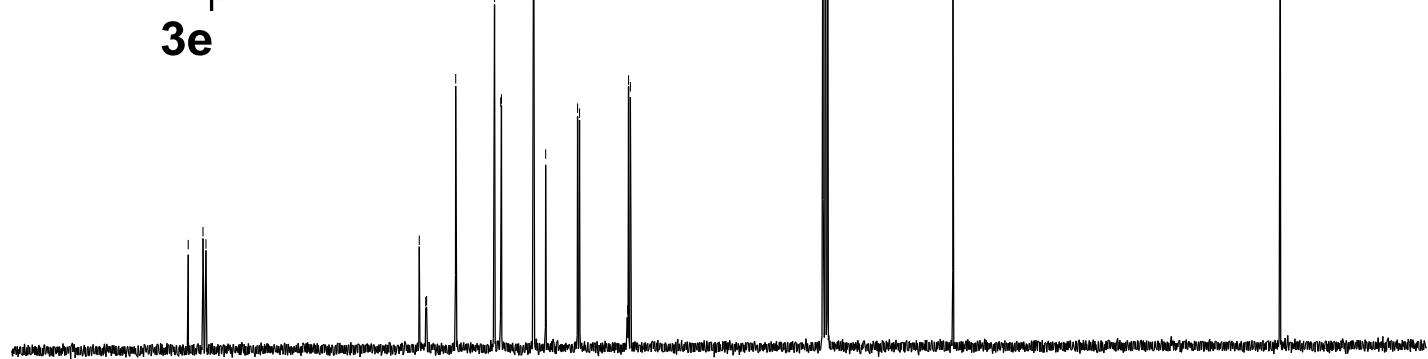

$\begin{array}{lllllllllllllllllll}180 & 170 & 160 & 150 & 140 & 130 & 120 & 110 & 100 \underset{\mathrm{f} 1(\mathrm{ppm})}{90} & 80 & 70 & 60 & 50 & 40 & 30 & 20 & 10 & 0\end{array}$ 
(6) The ${ }^{1} \mathrm{H}$ NMR and ${ }^{13} \mathrm{C}$ NMR spectrum for $\mathbf{3 f}$ (using $\mathrm{CDCl}_{3}$ as solvent)

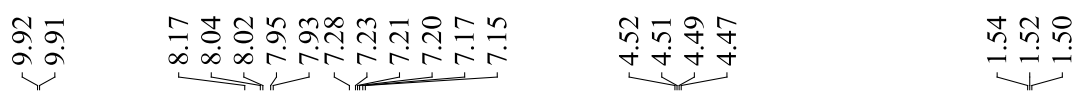

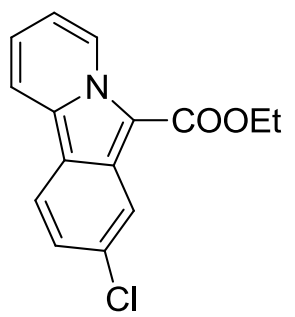

$3 f$

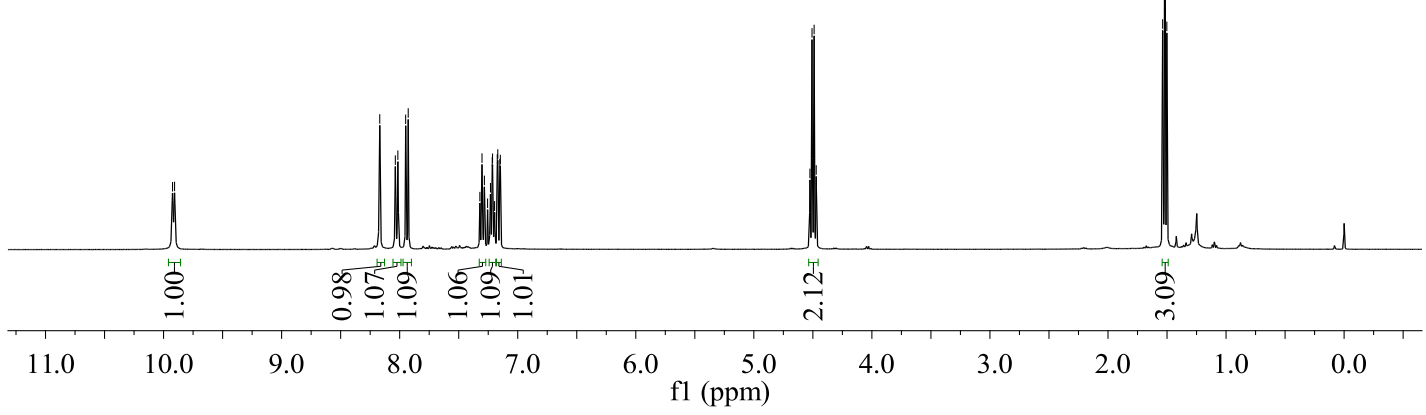

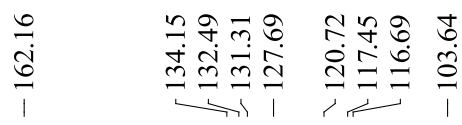
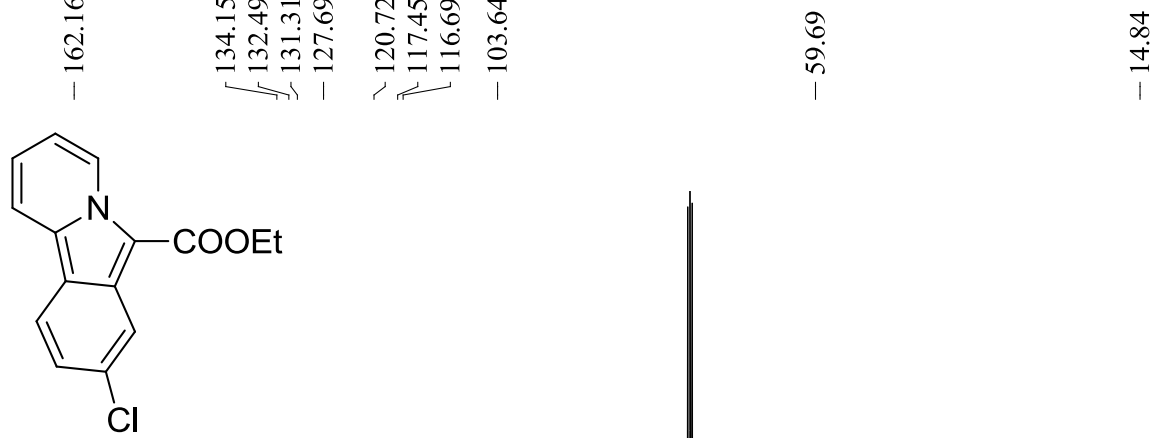

$3 f$

$\begin{array}{llllllllllllllllll}180 & 170 & 160 & 150 & 140 & 130 & 120 & 110 & 100 \underset{\mathrm{fl}(\mathrm{ppm})}{90} & 80 & 70 & 60 & 50 & 40 & 30 & 20 & 10 & 0\end{array}$ 
(7) The ${ }^{1} \mathrm{H}$ NMR and ${ }^{13} \mathrm{C}$ NMR spectrum for $\mathbf{3 g}$ (using $\mathrm{CDCl}_{3}$ as solvent)

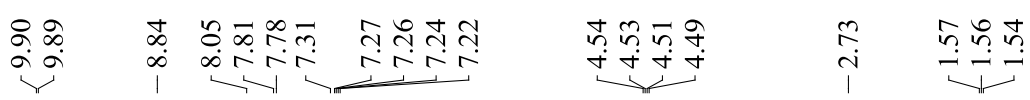

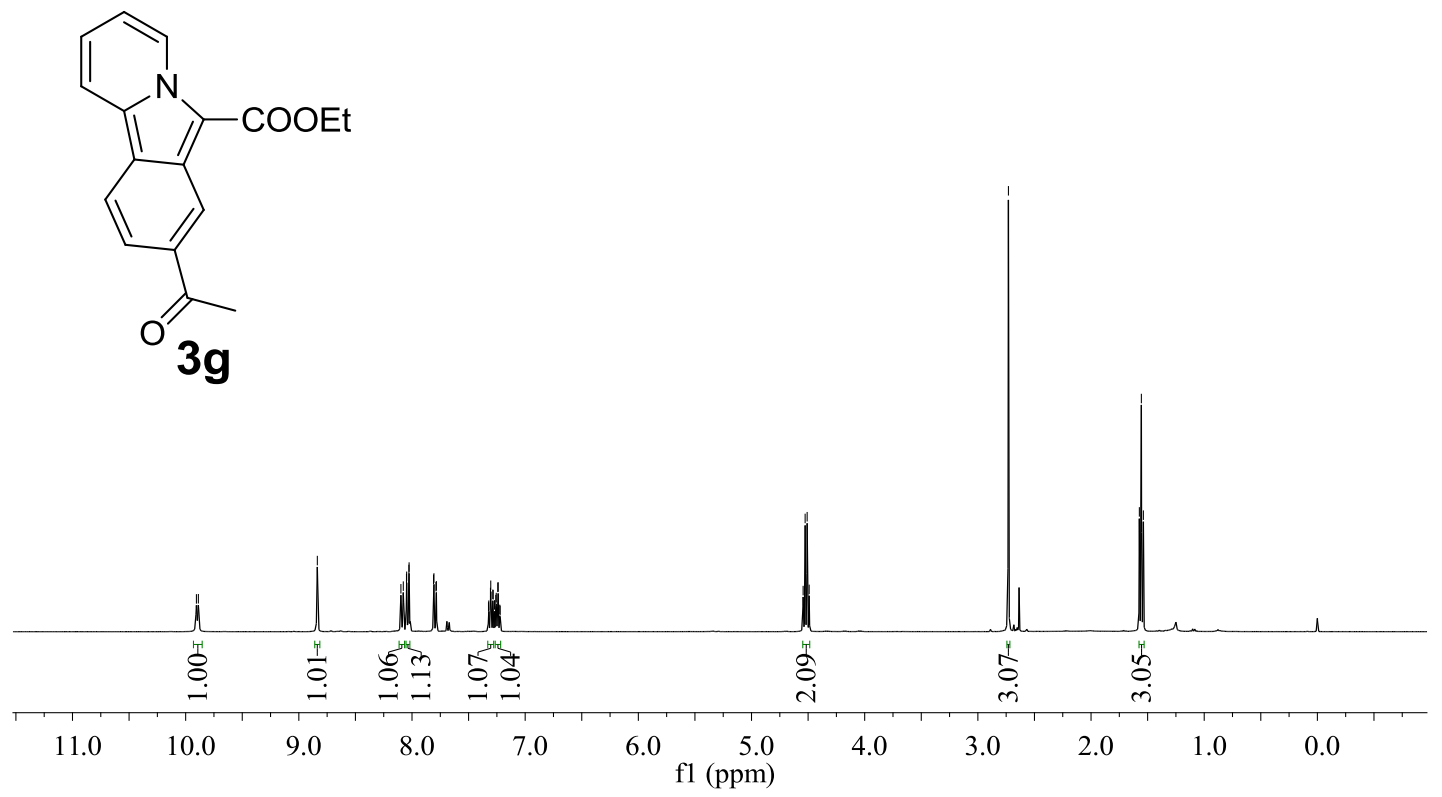

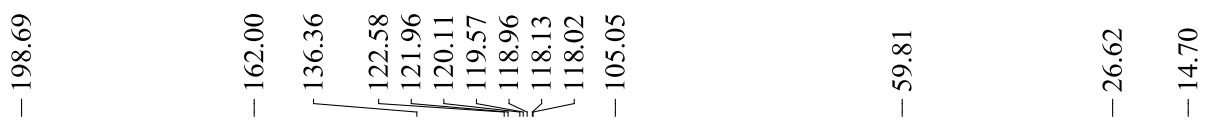

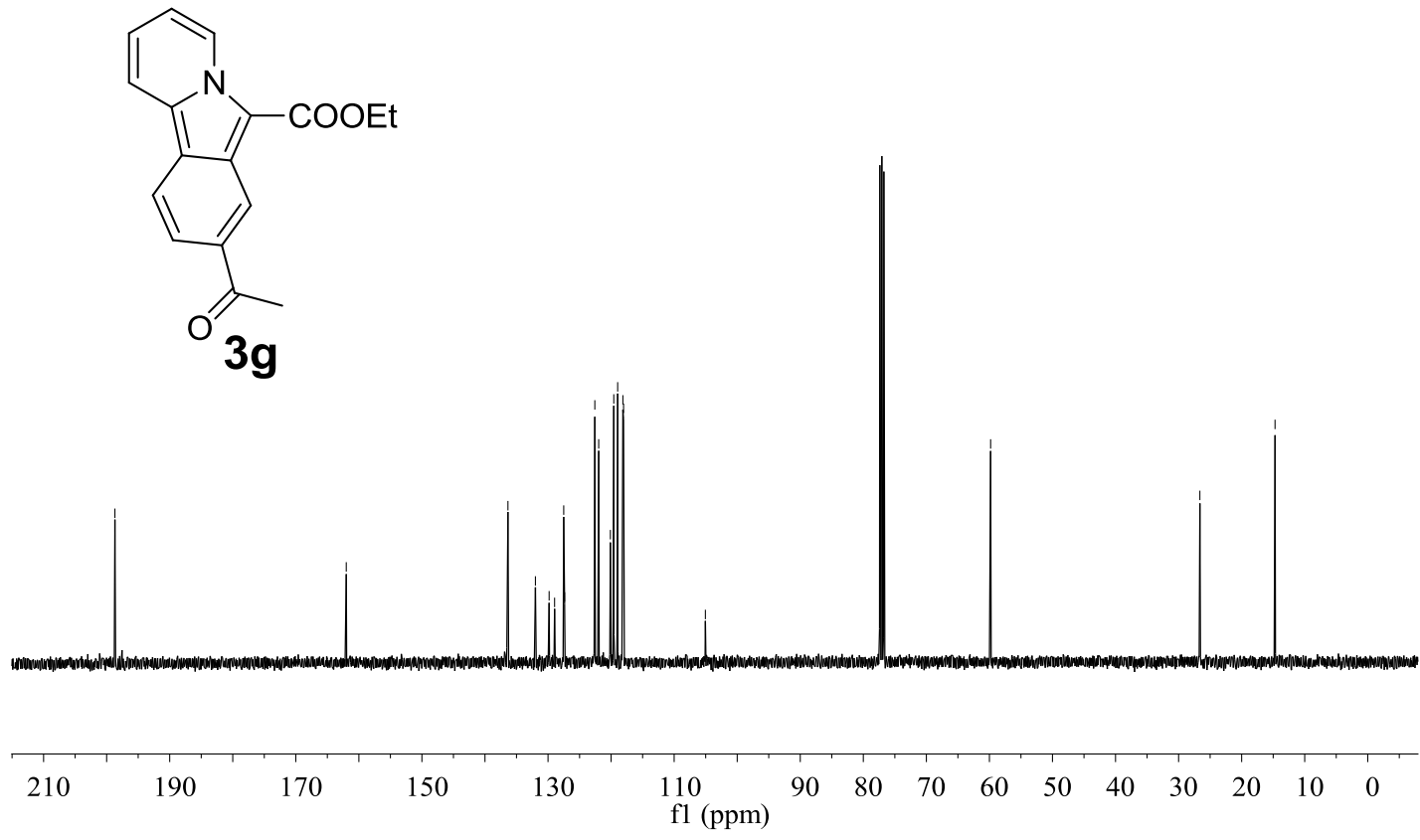


(8) The ${ }^{1} \mathrm{H}$ NMR and ${ }^{13} \mathrm{C}$ NMR spectrum for $\mathbf{3 h}$ (using $\mathrm{CDCl}_{3}$ as solvent)
ฉัँ
荧

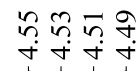
กุก<smiles>CCOC(=O)c1c2cc(C#N)ccc2c2ccccn12</smiles>

3h

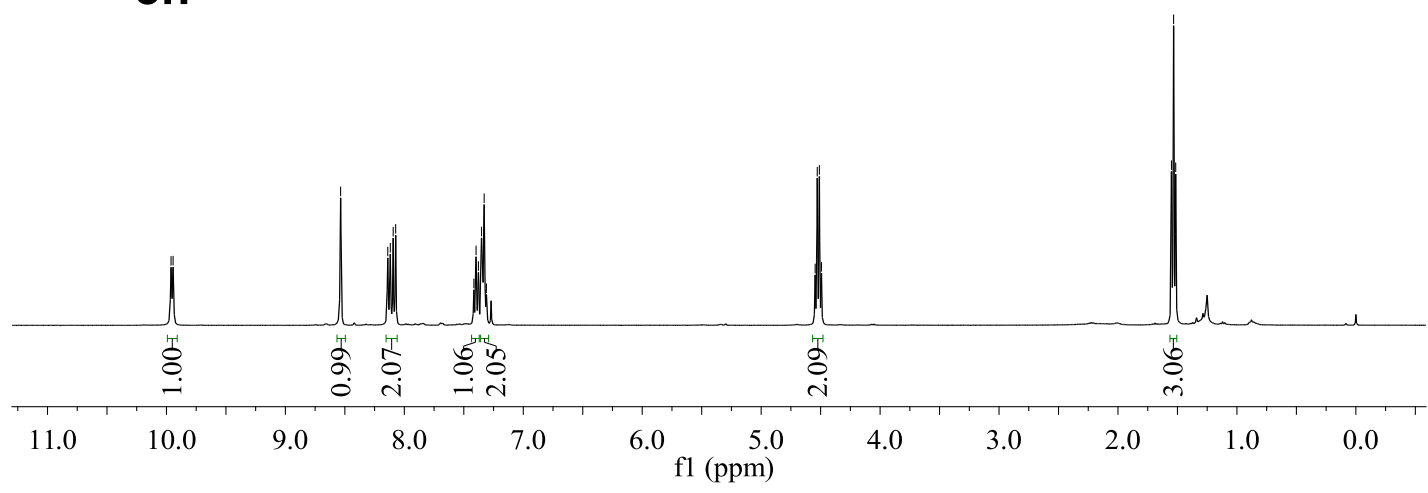

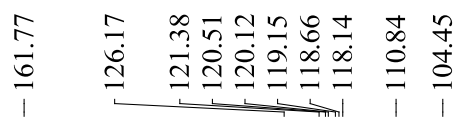
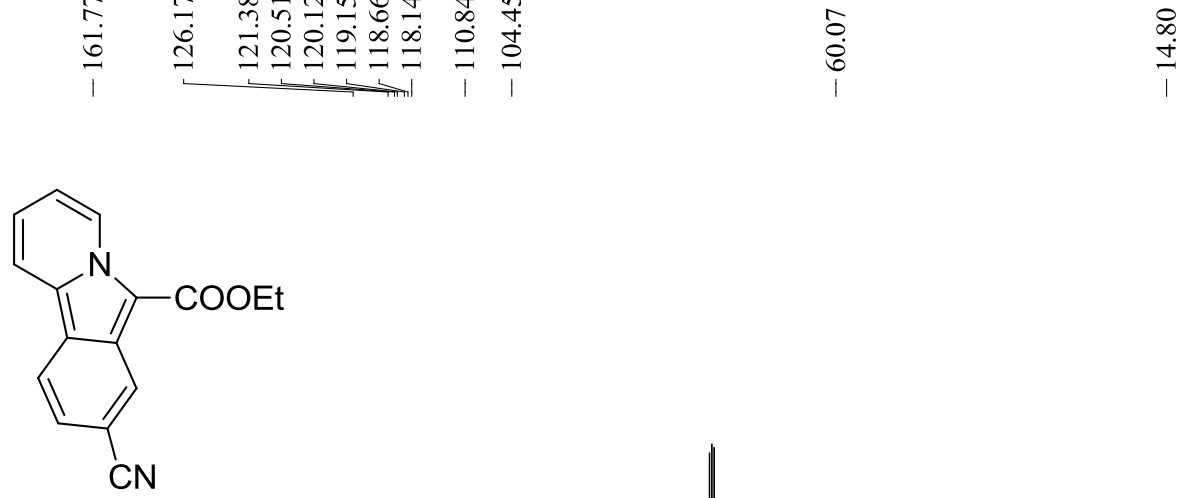

3h

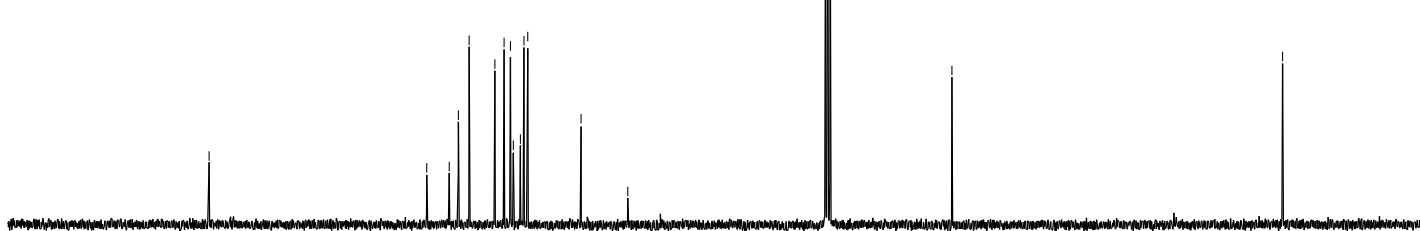

$\begin{array}{lllllllllllllllllll}180 & 170 & 160 & 150 & 140 & 130 & 120 & 110 & \begin{array}{c}100 \\ \mathrm{f} 1(\mathrm{ppm})\end{array} & \begin{array}{c}90 \\ (\mathrm{ppm})\end{array} & 70 & 60 & 50 & 40 & 30 & 20 & 10 & 0\end{array}$ 
(9) The ${ }^{1} \mathrm{H}$ NMR and ${ }^{13} \mathrm{C}$ NMR spectrum for $3 \mathbf{i}$ (using $\mathrm{CDCl}_{3}$ as solvent)

文<smiles>CCOC(=O)c1c2cc(C(F)(F)F)ccc2c2ccccn12</smiles>

$3 \mathbf{i}$

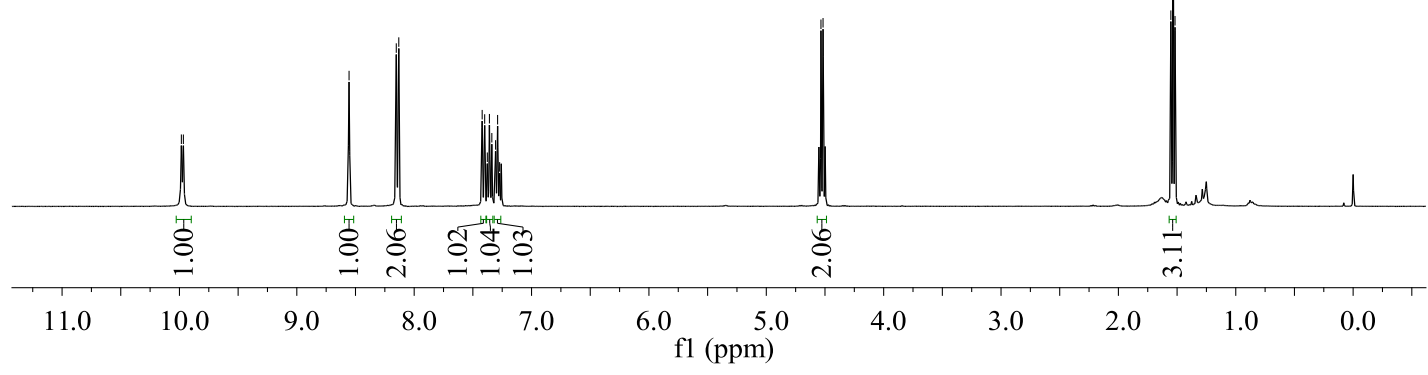

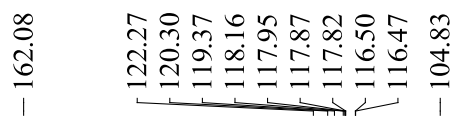
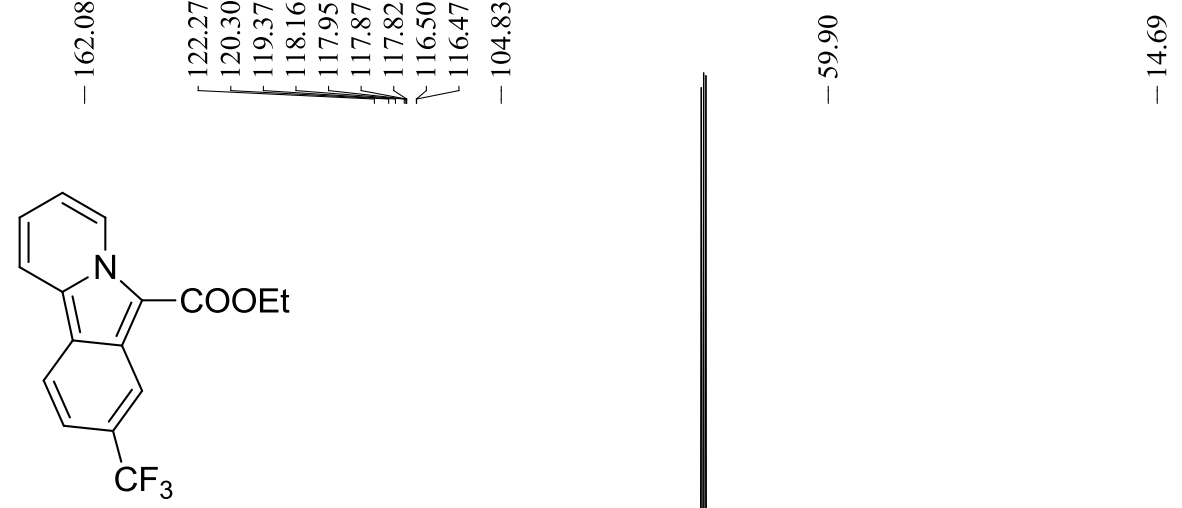

$3 \mathbf{i}$

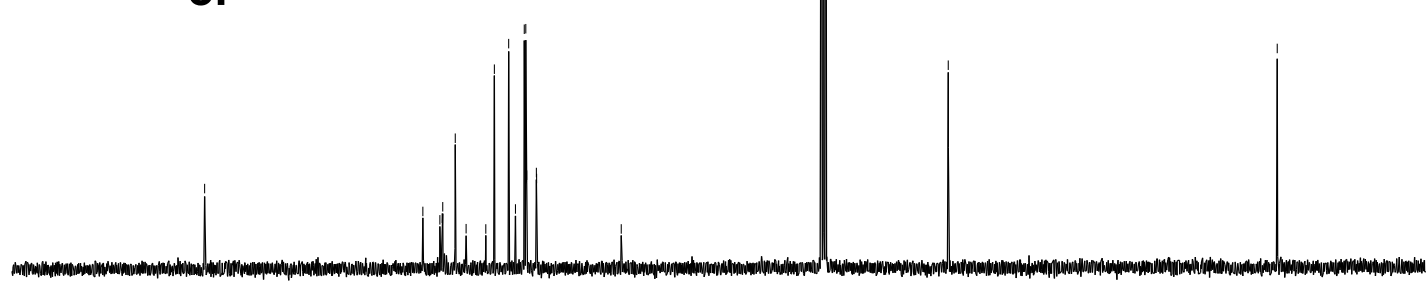

$\begin{array}{lllllllllllllllllll}180 & 170 & 160 & 150 & 140 & 130 & 120 & 110 & 100 \underset{\mathrm{f} 1(\mathrm{ppm})}{90} & 80 & 70 & 60 & 50 & 40 & 30 & 20 & 10 & 0\end{array}$ 
(10) The ${ }^{1} \mathrm{H}$ NMR and ${ }^{13} \mathrm{C}$ NMR spectrum for $\mathbf{3 j}$ (using $\mathrm{CDCl}_{3}$ as solvent)

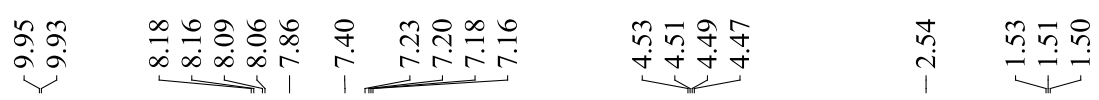<smiles>CCOC(=O)c1c2ccc(C)cc2c2ccccn12</smiles>

3j

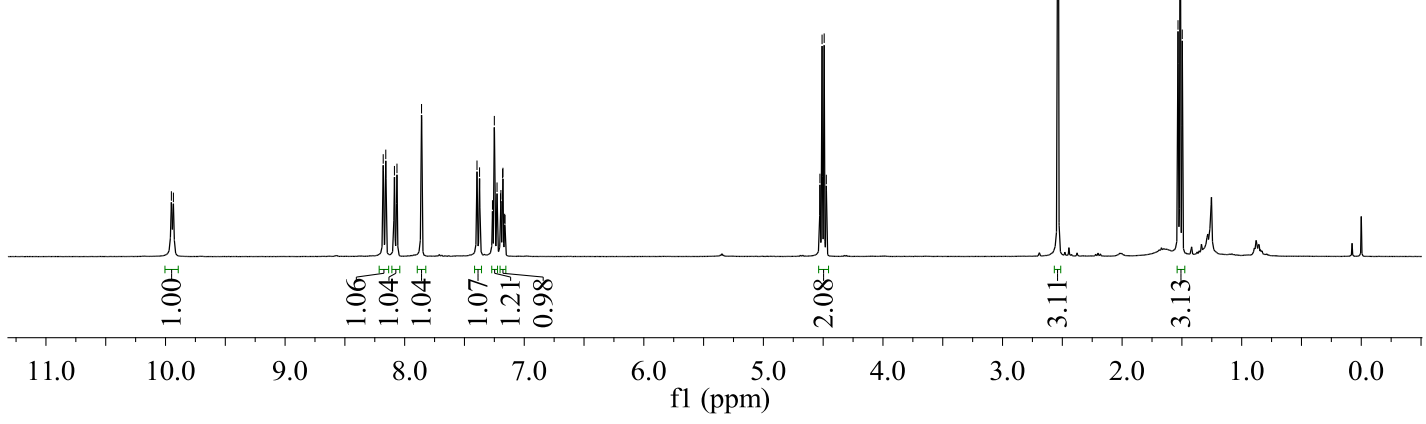

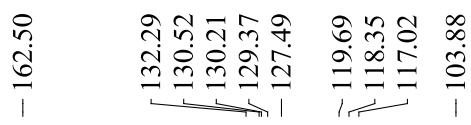

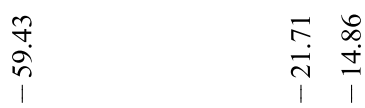<smiles>CCOC(=O)c1c2ccc(C)cc2c2ccccn12</smiles>

3j

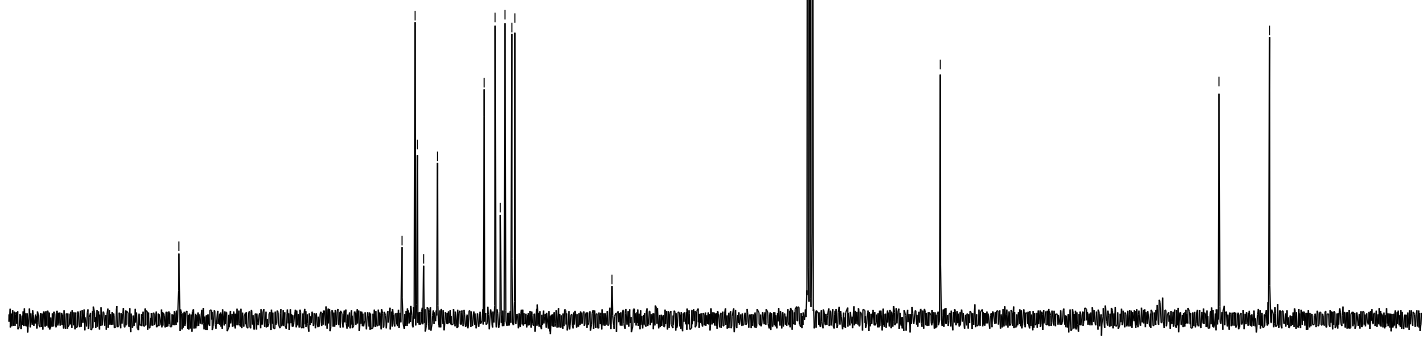

$\begin{array}{lllllllllllllllllll}180 & 170 & 160 & 150 & 140 & 130 & 120 & 110 & 100 \underset{\mathrm{f} 1 \underset{(\mathrm{ppm})}{90}}{80} & 70 & 60 & 50 & 40 & 30 & 20 & 10 & 0\end{array}$ 
(11) The ${ }^{1} \mathrm{H}$ NMR and ${ }^{13} \mathrm{C}$ NMR spectrum for $\mathbf{3 k}$ (using $\mathrm{CDCl}_{3}$ as solvent)

$$
\text { 每 }
$$<smiles>CCOC(=O)c1c2ccc(OC)cc2c2ccccn12</smiles>

3k

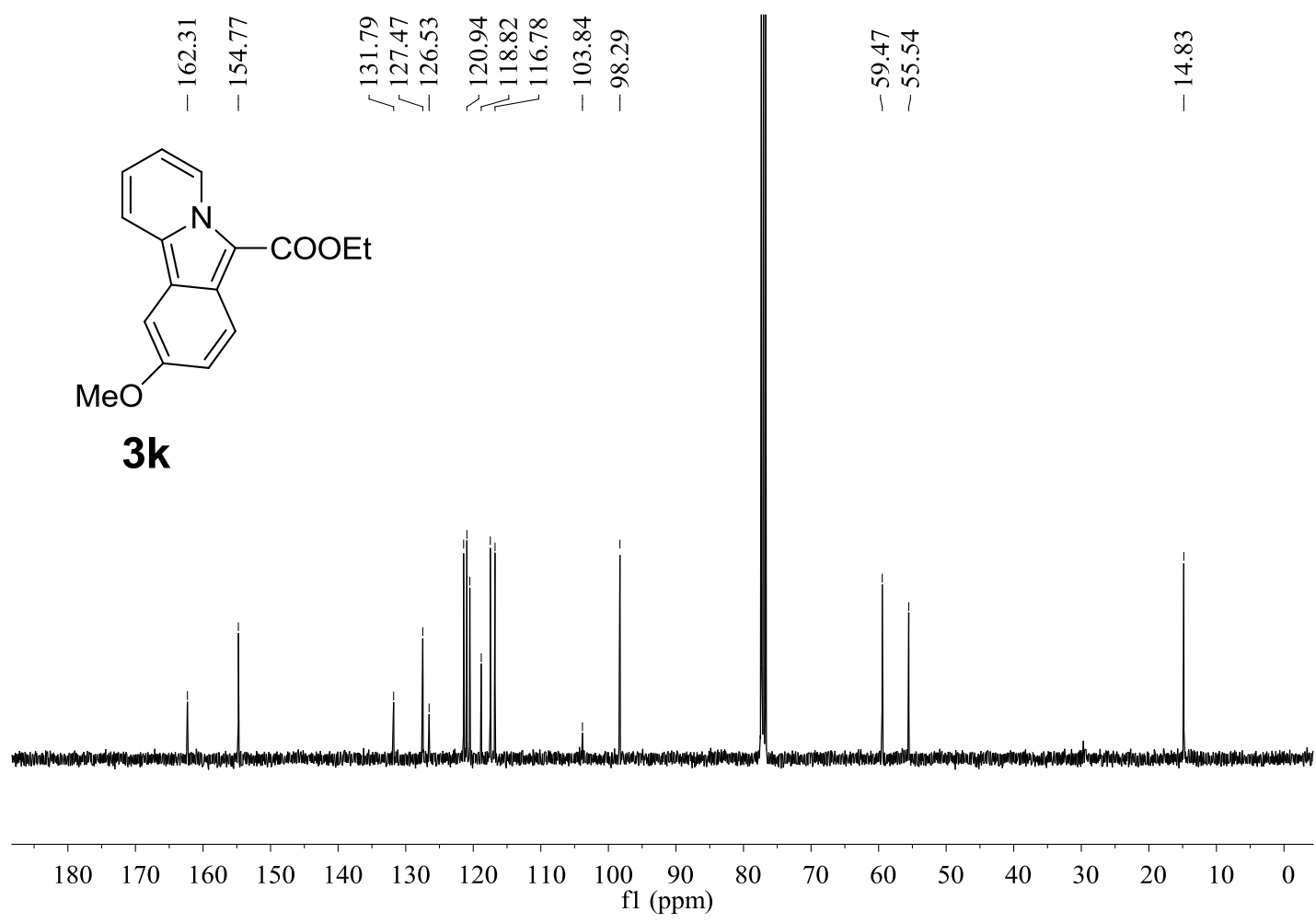


(12) The ${ }^{1} \mathrm{H}$ NMR and ${ }^{13} \mathrm{C}$ NMR spectrum for 31 (using $\mathrm{CDCl}_{3}$ as solvent)

$$
\text { 舟 }
$$<smiles>CCOC(=O)c1c2ccc(Cl)cc2c2ccccn12</smiles>

31

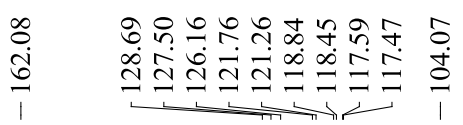

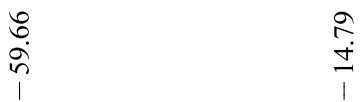<smiles>CCOC(=O)c1c2ccc(Cl)cc2c2ccccn12</smiles>

$\begin{array}{lllllllllllllllllll}180 & 170 & 160 & 150 & 140 & 130 & 120 & 110 & 100 \underset{\mathrm{f} 1}{(\mathrm{ppm})} & 90 & 80 & 70 & 60 & 50 & 40 & 30 & 20 & 10 & 0\end{array}$ 
(13) The ${ }^{1} \mathrm{H}$ NMR and ${ }^{13} \mathrm{C}$ NMR spectrum for $\mathbf{3 m}$ (using $\mathrm{CDCl}_{3}$ as solvent)

$$
\text { jå }
$$

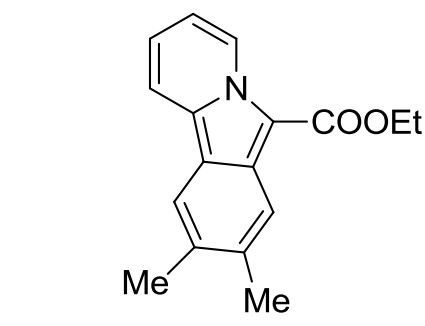

$3 \mathrm{~m}$

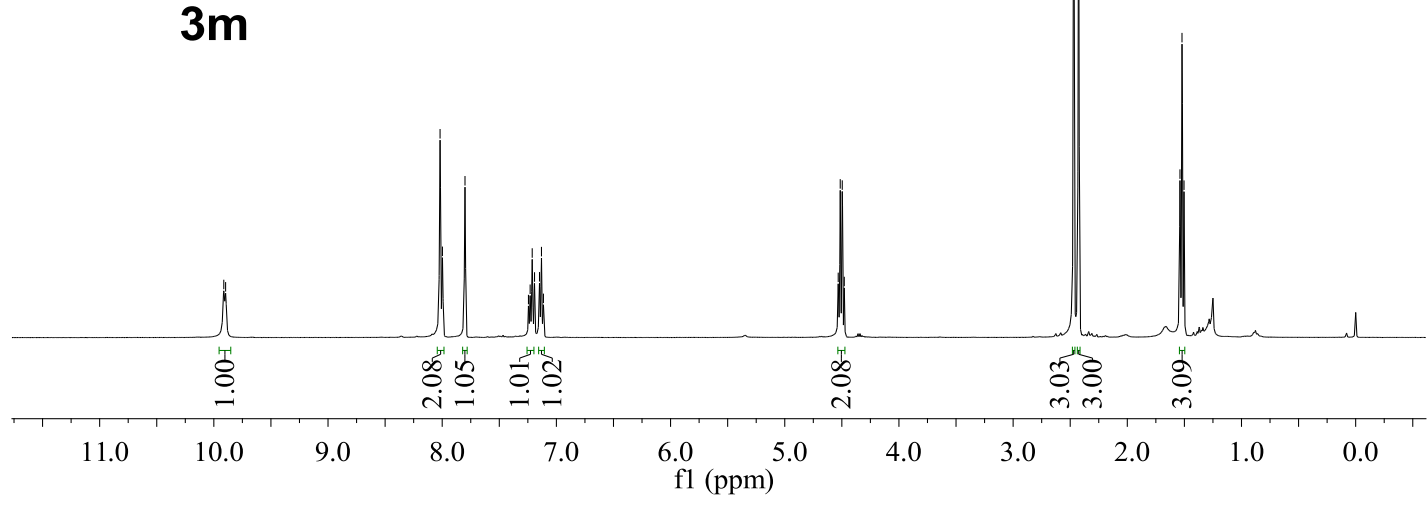

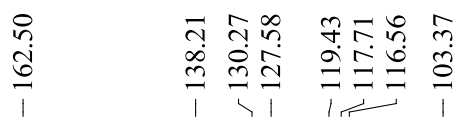
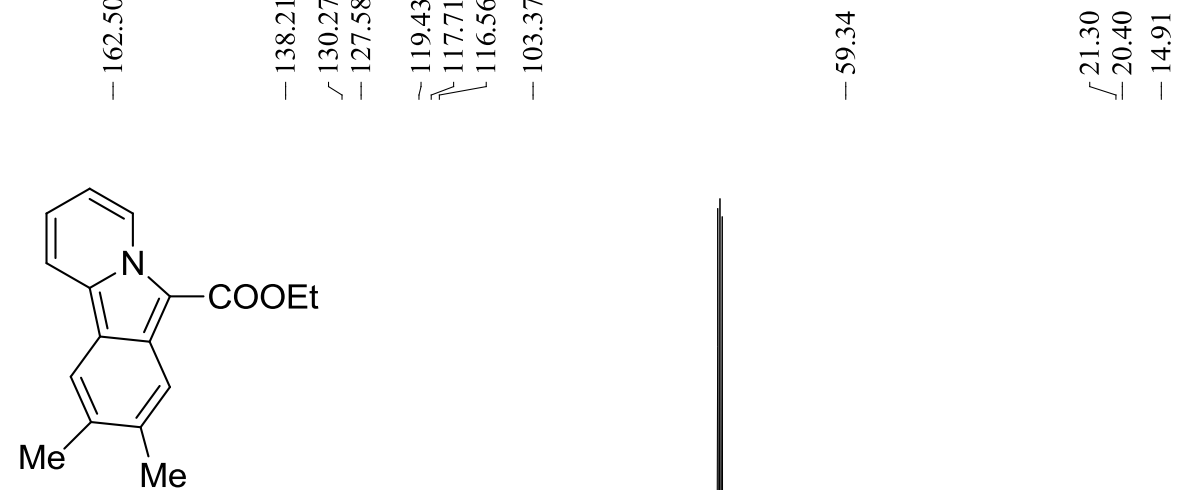

$3 \mathrm{~m}$

190

170

150

130

110

90

$\begin{array}{lllllll}60 & 50 & 40 & 30 & 20 & 10 & 0\end{array}$ 
(14) The ${ }^{1} \mathrm{H}$ NMR and ${ }^{13} \mathrm{C}$ NMR spectrum for $3 n$ (using $\mathrm{CDCl}_{3}$ as solvent)

Vl

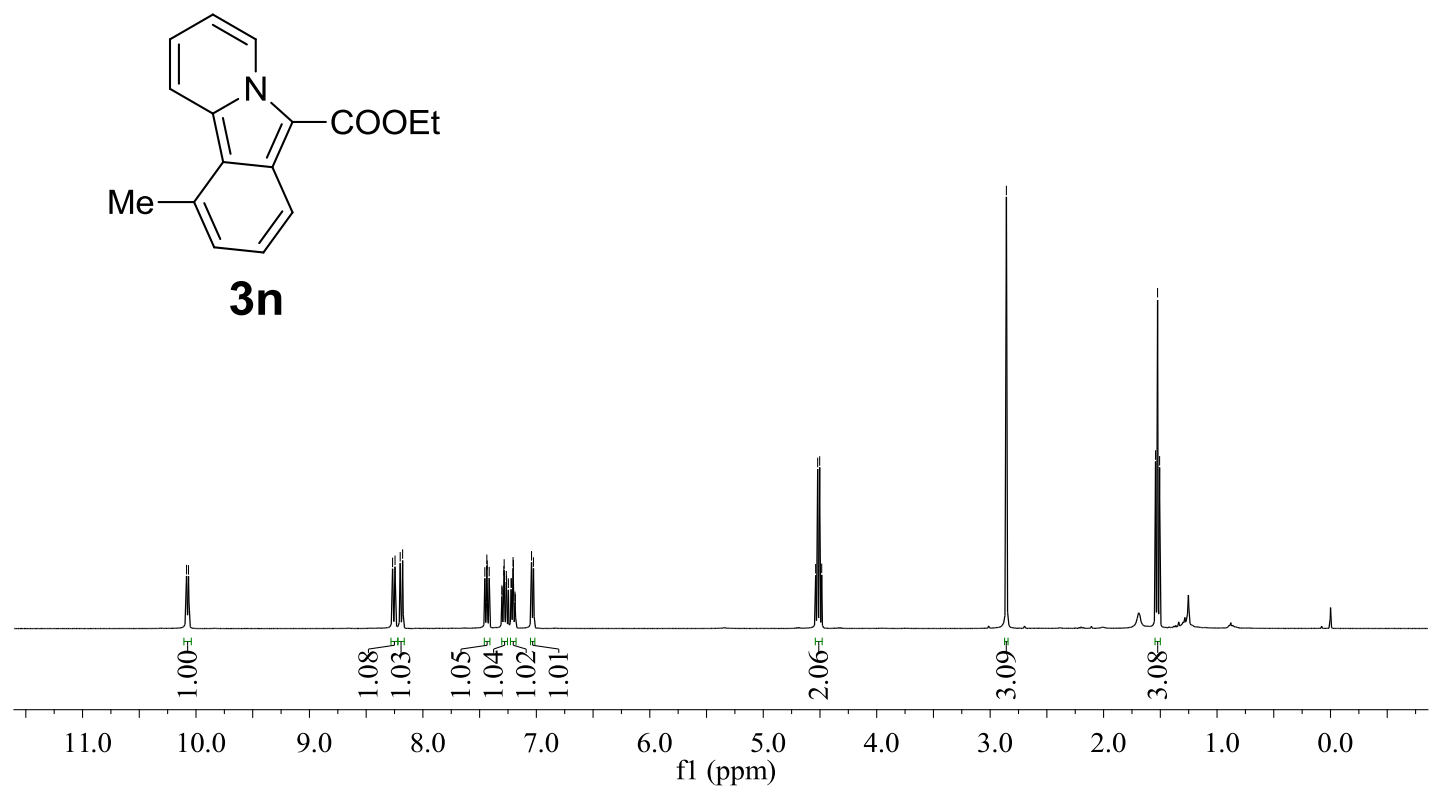

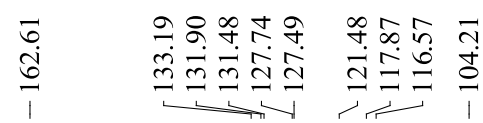

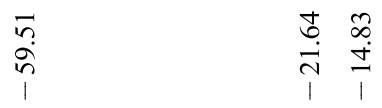<smiles>CCOC(=O)c1c2cccc(C)c2c2ccccn12</smiles>

$3 n$

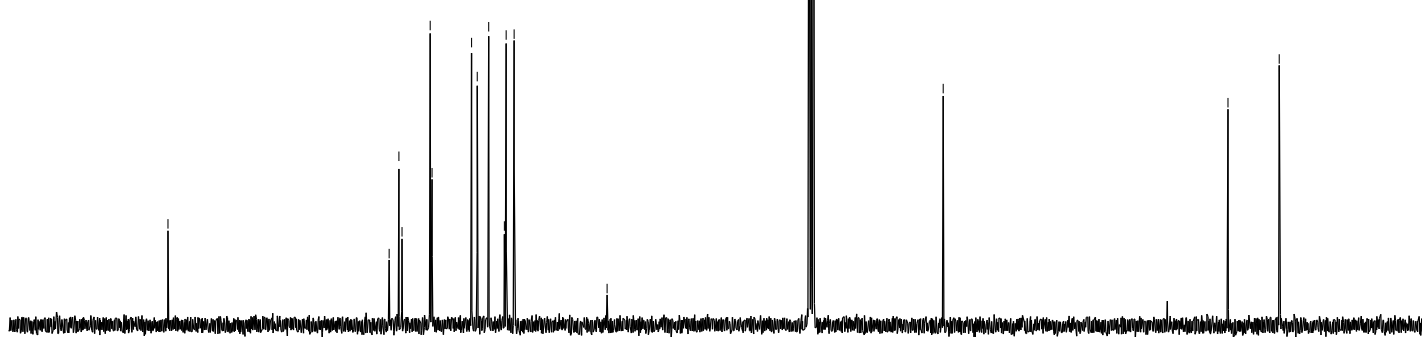

$\begin{array}{lllllllllllllllllll}180 & 170 & 160 & 150 & 140 & 130 & 120 & 110 & 100 & 90 & 80 & 70 & 60 & 50 & 40 & 30 & 20 & 10 & 0\end{array}$ 
(15) The ${ }^{1} \mathrm{H}$ NMR and ${ }^{13} \mathrm{C}$ NMR spectrum for 30 (using $\mathrm{CDCl}_{3}$ as solvent)

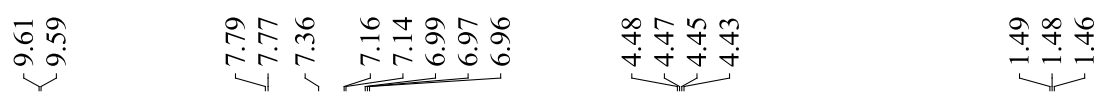<smiles>CCOC(=O)c1c2sccc2c2ccccn12</smiles>

30

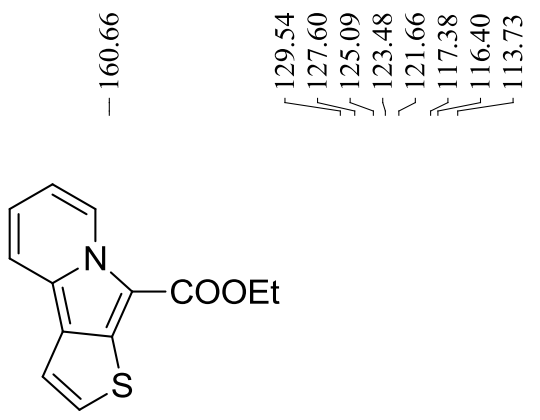

30
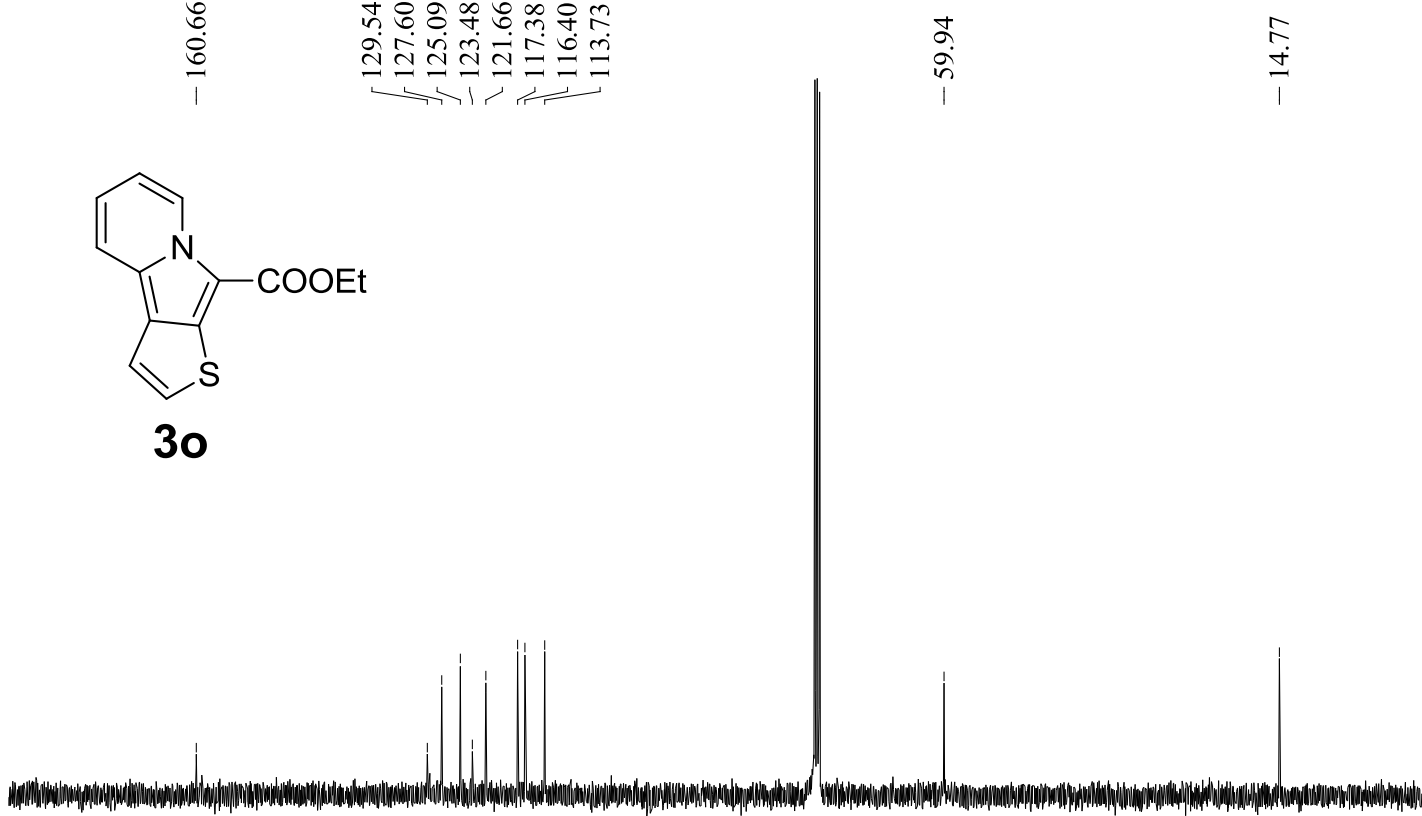

$\begin{array}{lllllllllllllllllll}180 & 170 & 160 & 150 & 140 & 130 & 120 & 110 & 100 \underset{\mathrm{f} 1}{(\mathrm{ppm})} & 80 & 70 & 60 & 50 & 40 & 30 & 20 & 10 & 0\end{array}$ 
(16) The ${ }^{1} \mathrm{H}$ NMR and ${ }^{13} \mathrm{C}$ NMR spectrum for $3 p$ (using $\mathrm{CDCl}_{3}$ as solvent)

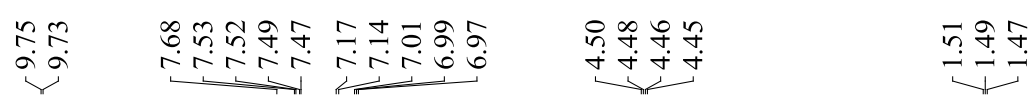<smiles>CCOC(=O)c1c2ccsc2c2ccccn12</smiles>
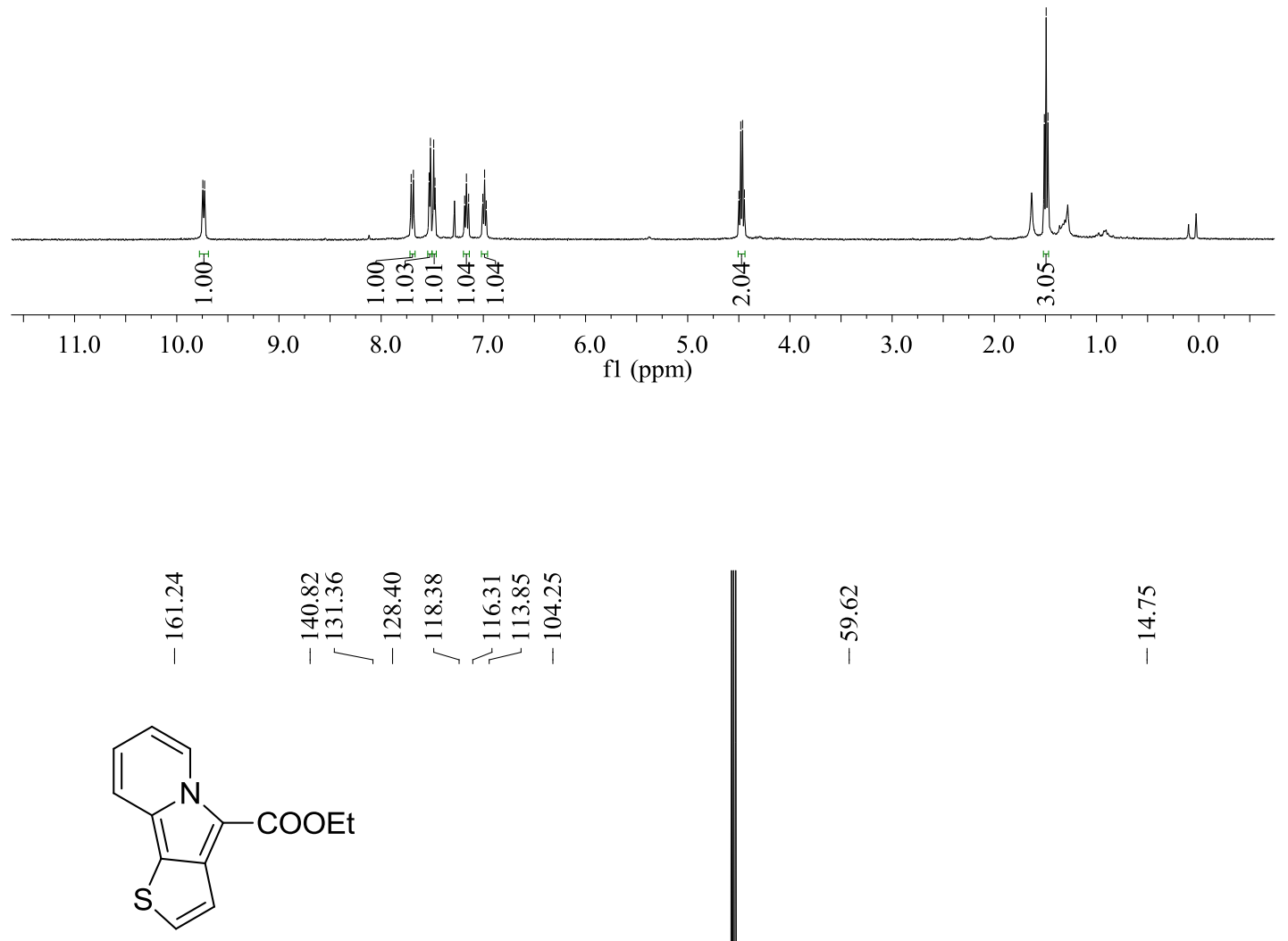

$3 p$

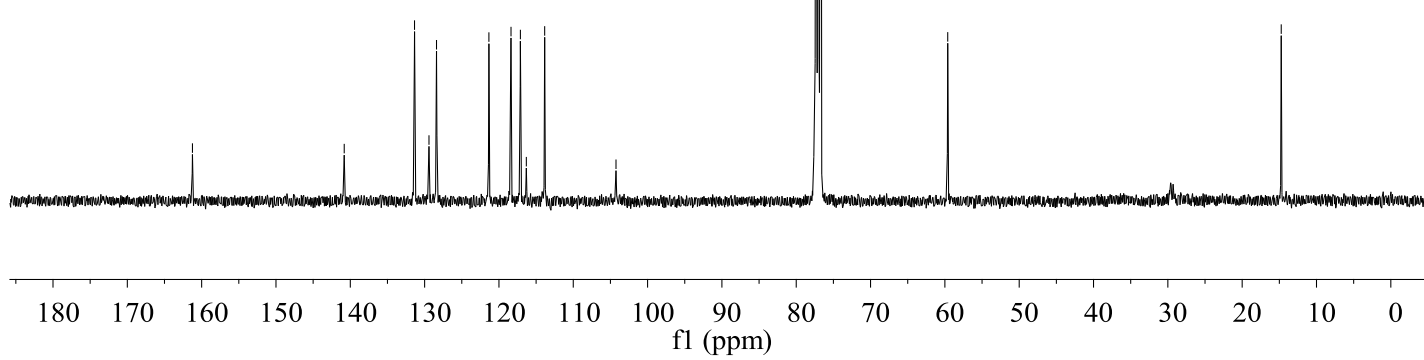


(17) The ${ }^{1} \mathrm{H}$ NMR and ${ }^{13} \mathrm{C}$ NMR spectrum for $\mathbf{3 q}$ (using $\mathrm{CDCl}_{3}$ as solvent)

$$
\text { 冓 我 }
$$<smiles>CCOC(=O)c1c2ccccc2c2ccc(C)cn12</smiles>

$3 q$

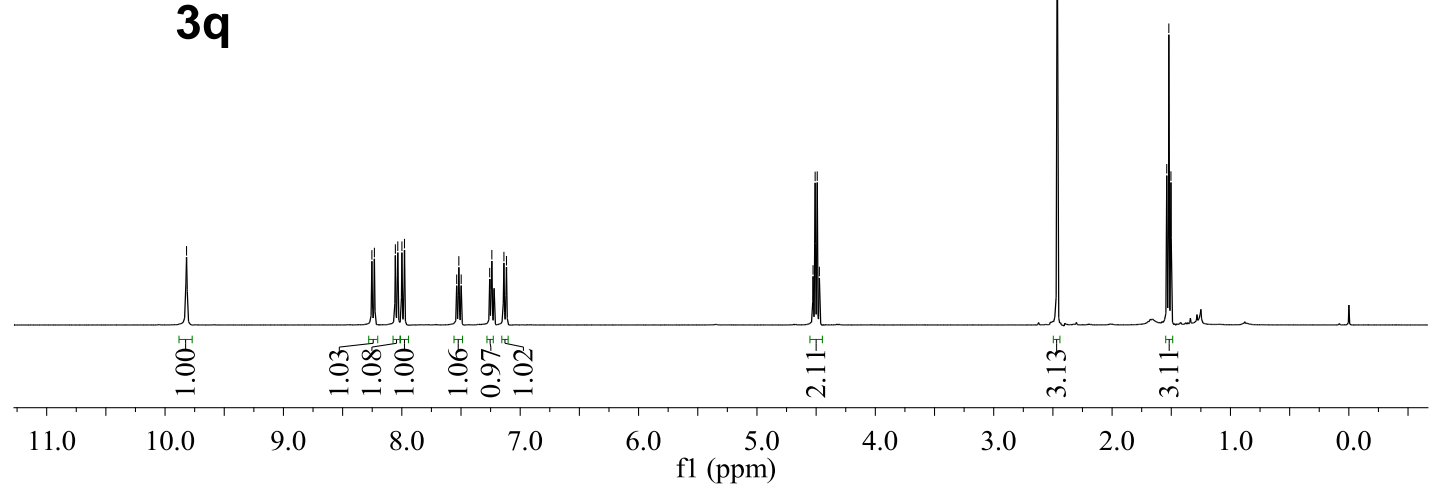

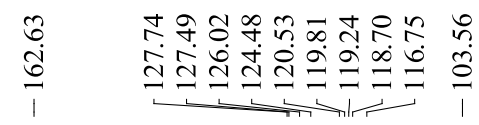<smiles>CCOC(=O)c1c2ccccc2c2ccc(C)cn12</smiles>

$3 q$ ì

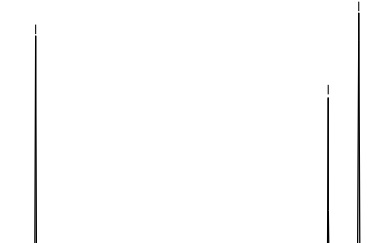


(18) The ${ }^{1} \mathrm{H}$ NMR and ${ }^{13} \mathrm{C}$ NMR spectrum for $3 \mathbf{r}$ (using $\mathrm{CDCl}_{3}$ as solvent)

$$
\text { î. }
$$<smiles>CCOC(=O)c1c2ccccc2c2ccc(OC)cn12</smiles>

$3 r$

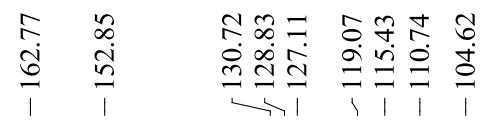

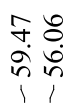

$\stackrel{\substack{\infty \\ \dot{ \pm}}}{\mid}$<smiles>CCOC(=O)c1c2ccccc2c2ccc(OC)cn12</smiles>

$3 r$

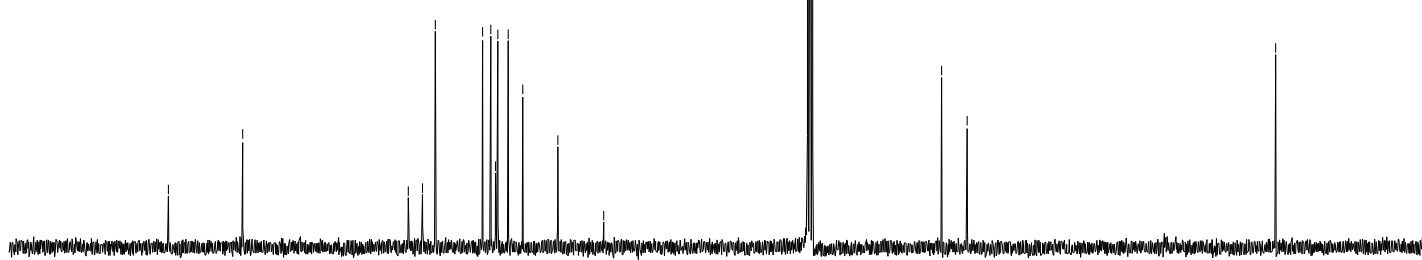

$\begin{array}{lllllllllllllllllll}180 & 170 & 160 & 150 & 140 & 130 & 120 & 110 & 100 & 90 & 80 & 70 & 60 & 50 & 40 & 30 & 20 & 10 & 0\end{array}$ 
(19) The ${ }^{1} \mathrm{H}$ NMR and ${ }^{13} \mathrm{C}$ NMR spectrum for $3 \mathbf{s}$ (using $\mathrm{CDCl}_{3}$ as solvent)

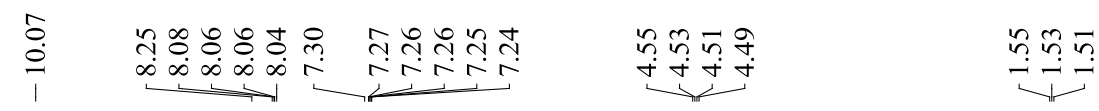<smiles>CCOC(=O)c1c2ccccc2c2ccc(Cl)cn12</smiles>

$3 s$

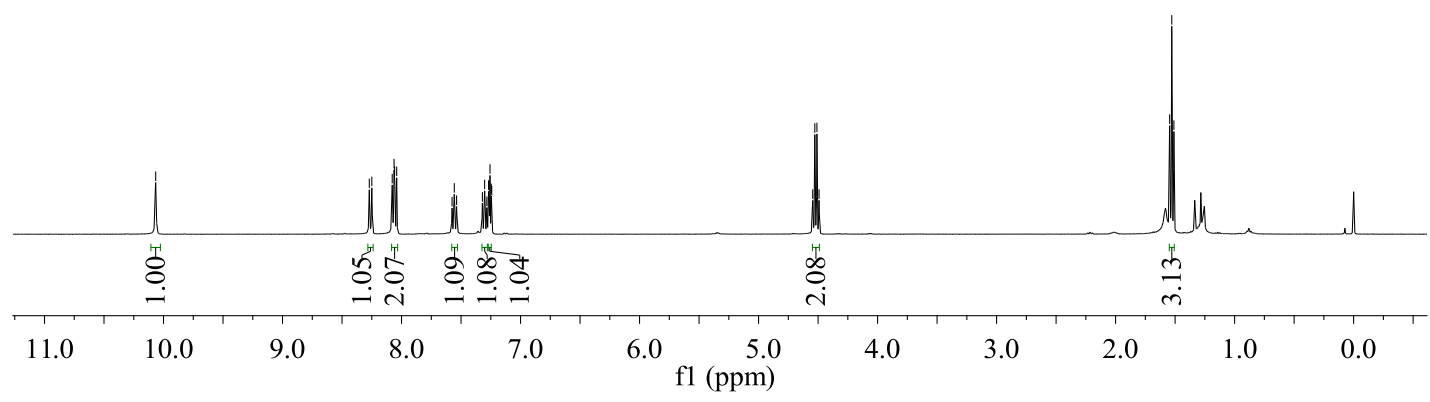

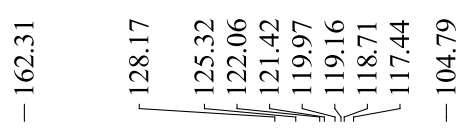

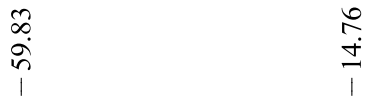<smiles>CCOC(=O)c1c2ccccc2c2ccc(Cl)cn12</smiles>

$3 s$

$\begin{array}{lllllllllllllllllll}180 & 170 & 160 & 150 & 140 & 130 & 120 & 110 & 100 & 90 & 80 & 70 & 60 & 50 & 40 & 30 & 20 & 10 & 0\end{array}$ 
(20) The ${ }^{1} \mathrm{H}$ NMR and ${ }^{13} \mathrm{C}$ NMR spectrum for $\mathbf{3 t}$ (using $\mathrm{CDCl}_{3}$ as solvent)

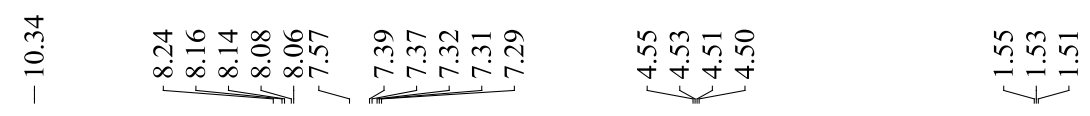<smiles>CCOC(=O)c1c2ccccc2c2ccc(C(F)(F)F)cn12</smiles>

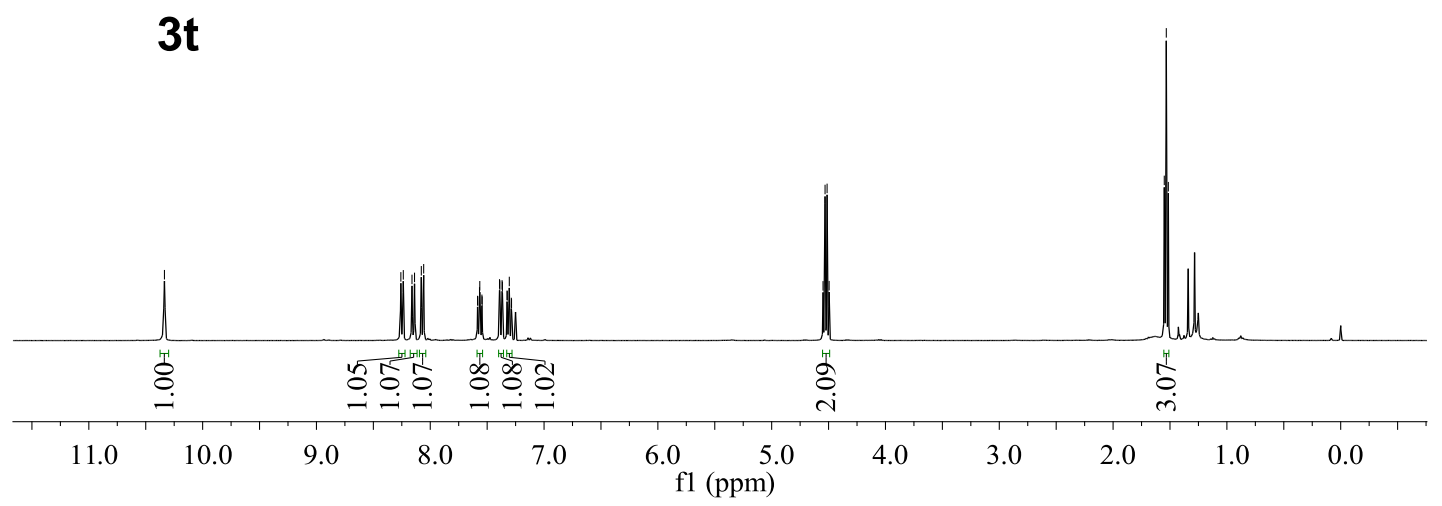

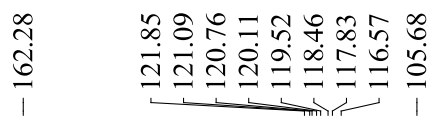
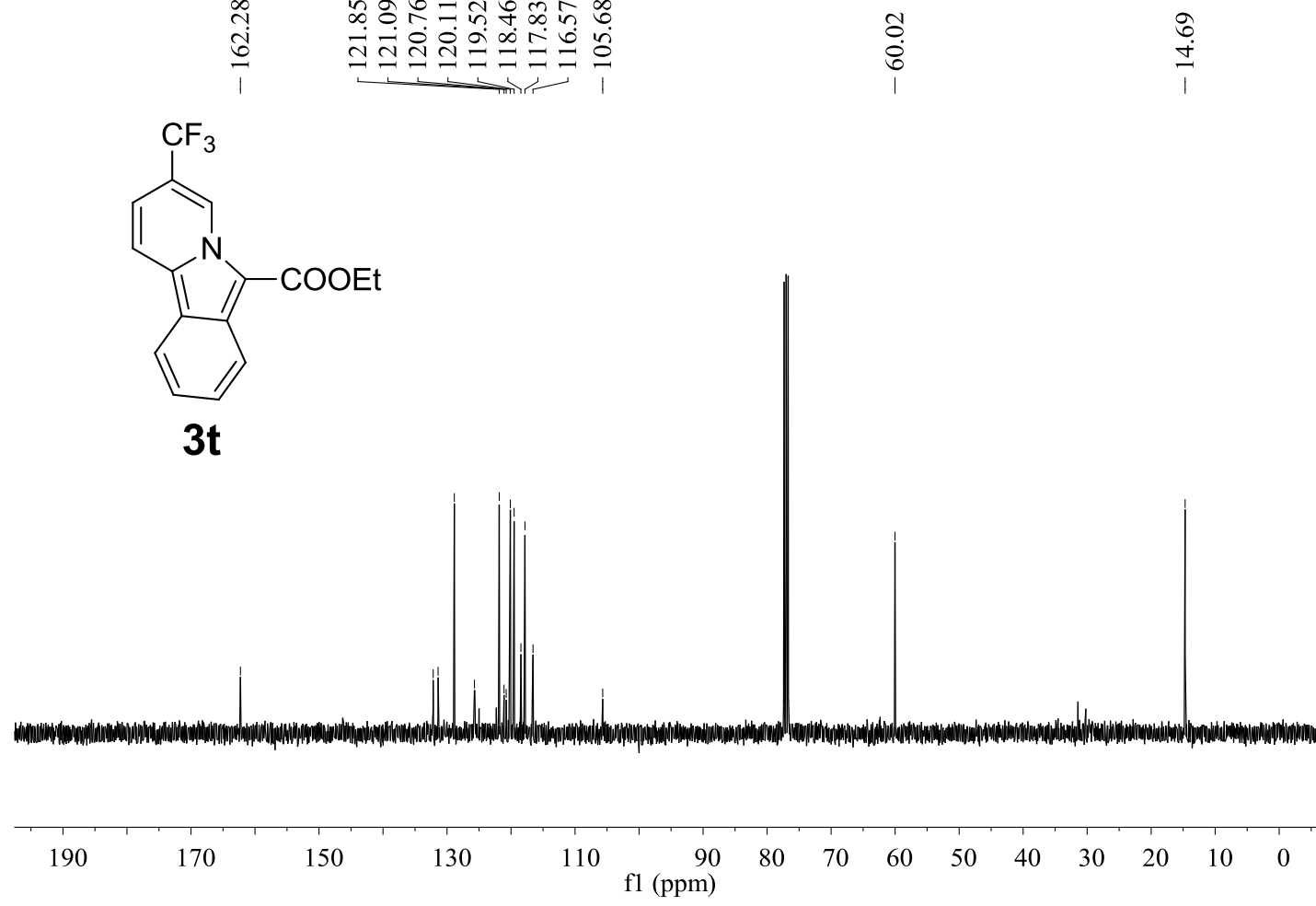
(21) The ${ }^{1} \mathrm{H}$ NMR and ${ }^{13} \mathrm{C}$ NMR spectrum for $3 \mathbf{u}$ (using $\mathrm{CDCl}_{3}$ as solvent)

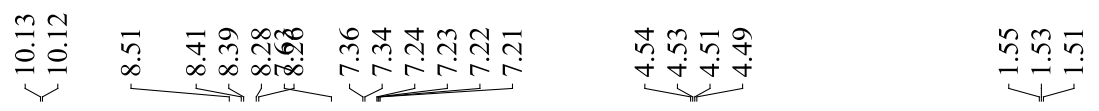<smiles>CCOC(=O)c1c2ccccc2c2ncccn12</smiles>

$3 u$

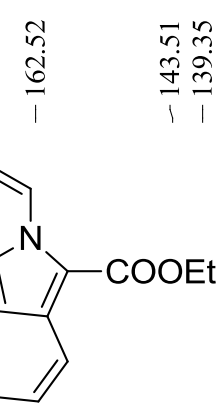

$3 u$

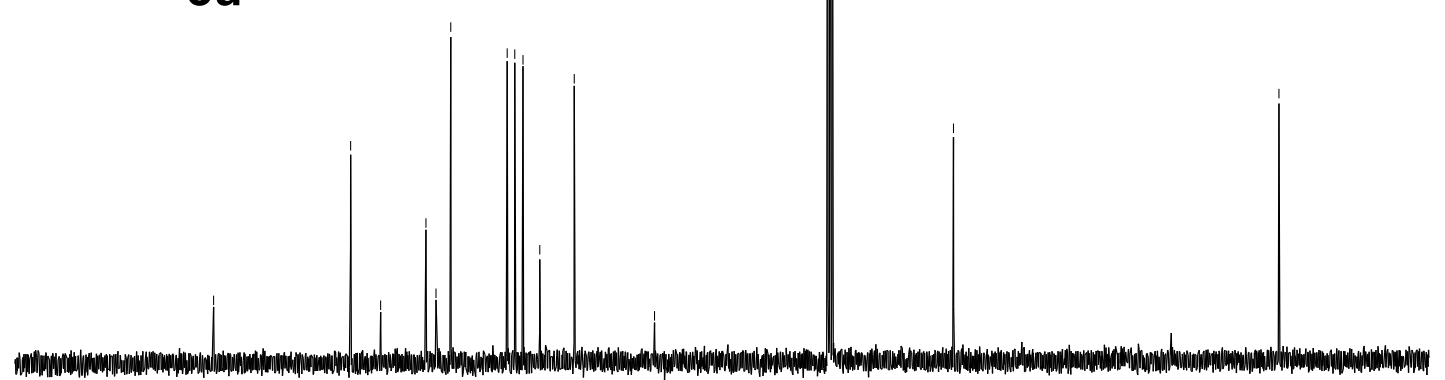

$\begin{array}{llllllllllllllllllll}190 & 180 & 170 & 160 & 150 & 140 & 130 & 120 & 110 & 100 & 90 & 80 & 70 & 60 & 50 & 40 & 30 & 20 & 10 & 0\end{array}$ 
(22) The ${ }^{1} \mathrm{H}$ NMR and ${ }^{13} \mathrm{C}$ NMR spectrum for $\mathbf{3 v}$ (using $\mathrm{CDCl}_{3}$ as solvent)

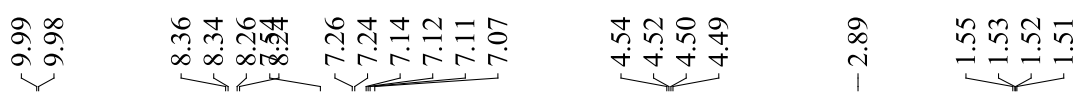<smiles>CCOC(=O)c1c2ccccc2c2c(C)cccn12</smiles>

$3 \mathbf{v}$

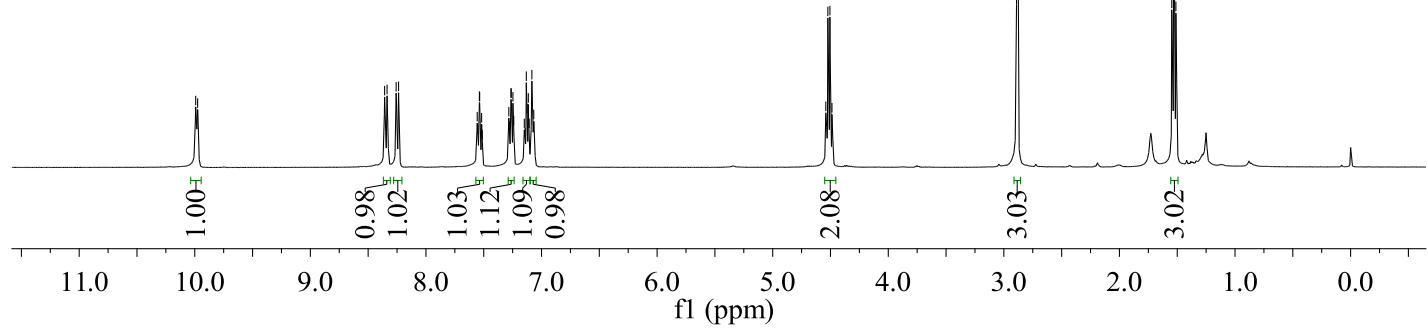

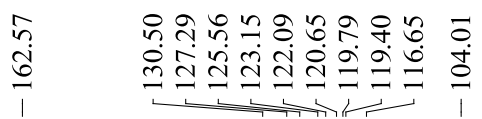

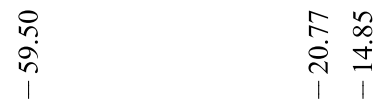<smiles>CCOC(=O)c1c2ccccc2c2c(C)cccn12</smiles>

$3 v$

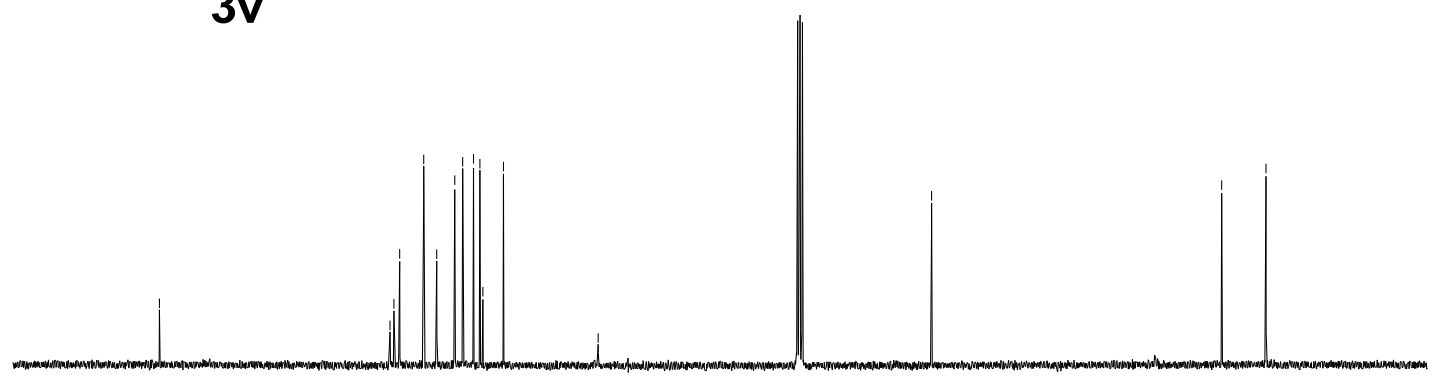

$\begin{array}{lllllllllllllllllll}180 & 170 & 160 & 150 & 140 & 130 & 120 & 110 & 100 & 90 & 80 & 70 & 60 & 50 & 40 & 30 & 20 & 10 & 0\end{array}$ 
(23) The ${ }^{1} \mathrm{H}$ NMR and ${ }^{13} \mathrm{C}$ NMR spectrum for $\mathbf{3 w}$ (using $\mathrm{CDCl}_{3}$ as solvent)
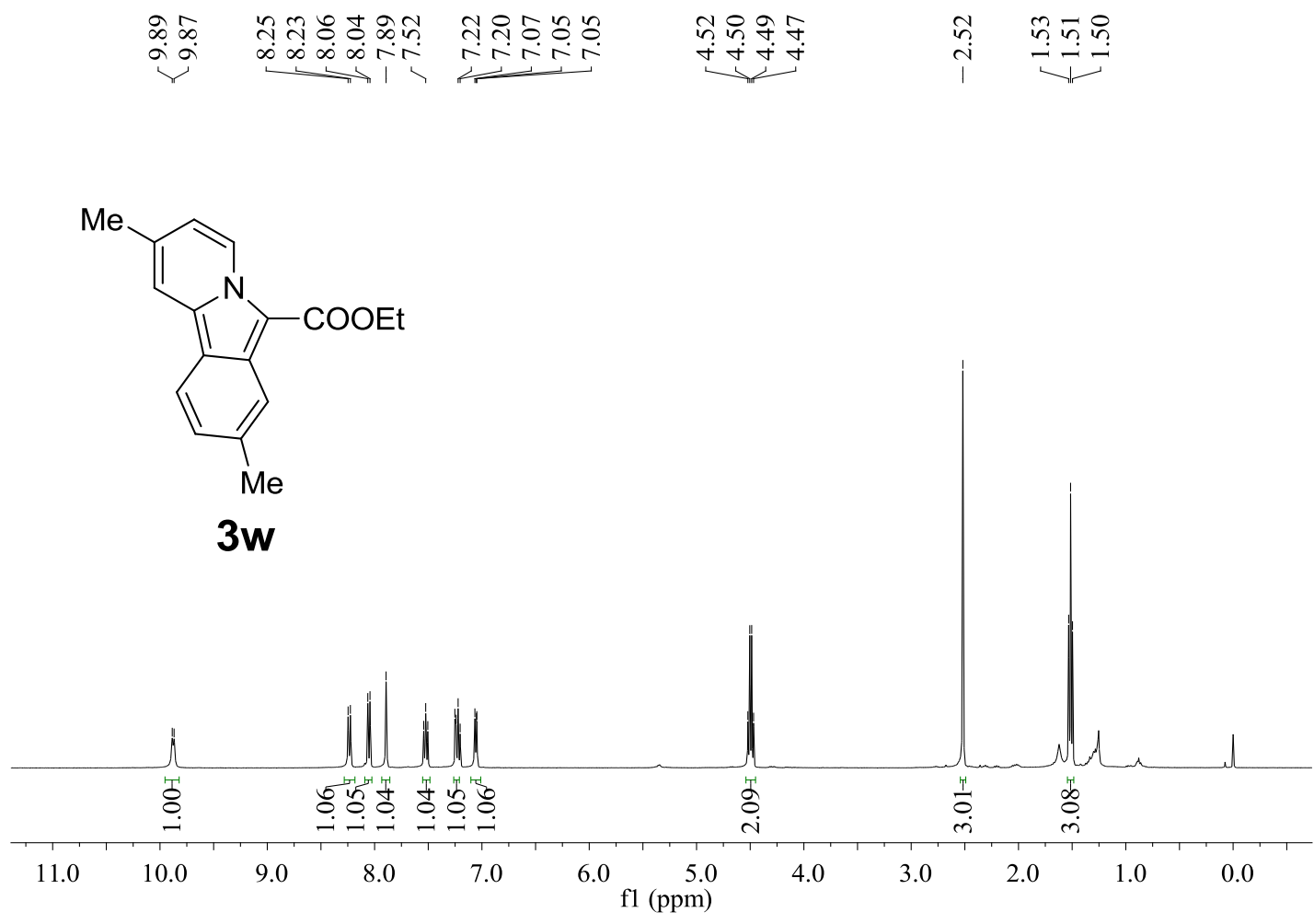

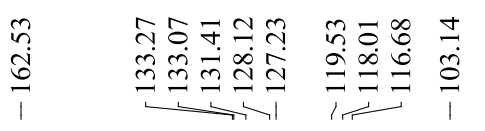
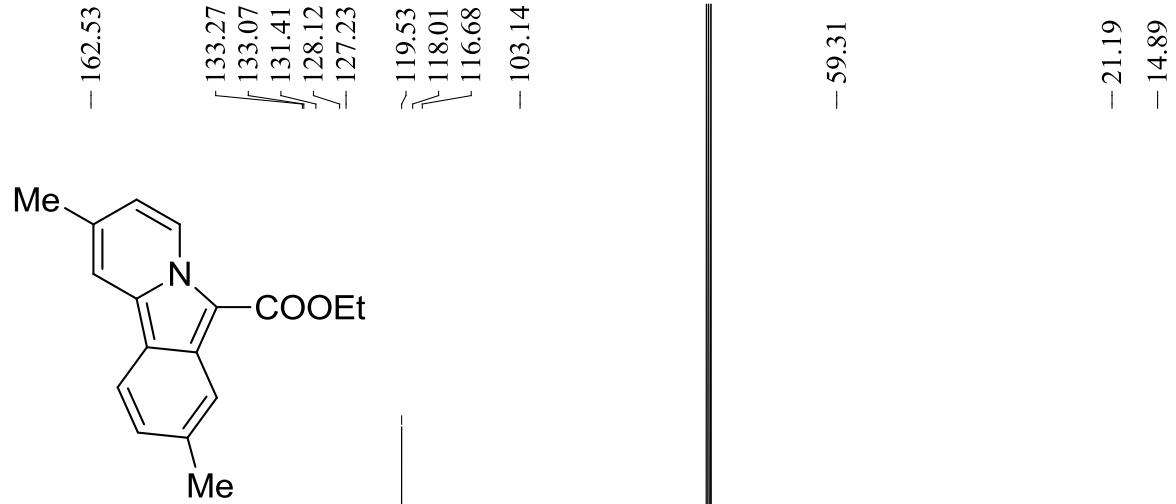

$3 w$

$\begin{array}{lllllllllllllllllll}180 & 170 & 160 & 150 & 140 & 130 & 120 & 110 & 100 & 90 & 80 & 70 & 60 & 50 & 40 & 30 & 20 & 10 & 0\end{array}$ 
(24) The ${ }^{1} \mathrm{H}$ NMR and ${ }^{13} \mathrm{C}$ NMR spectrum for $\mathbf{3 x}$ (using $\mathrm{CDCl}_{3}$ as solvent)

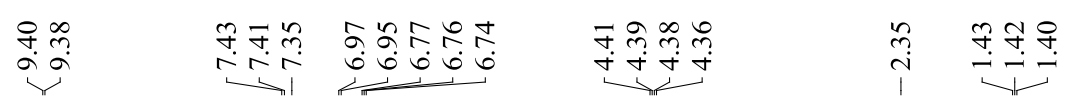<smiles>CCOC(=O)c1cc(C)c2ccccn12</smiles>

$3 x$

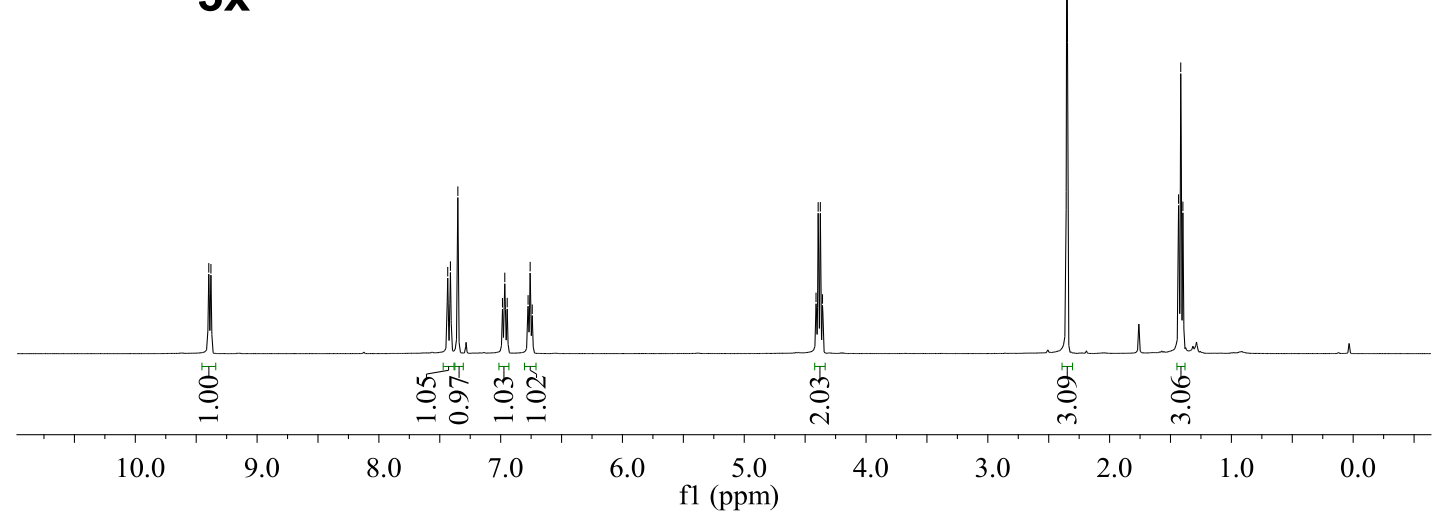

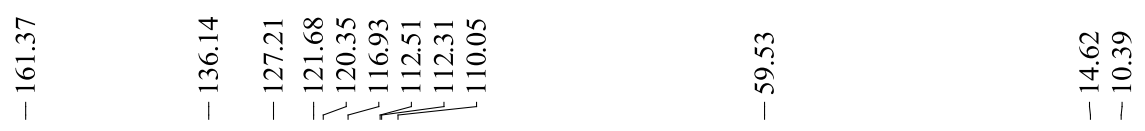

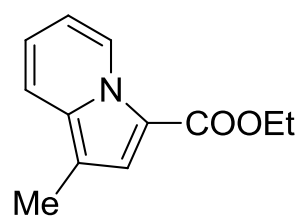

$3 x$

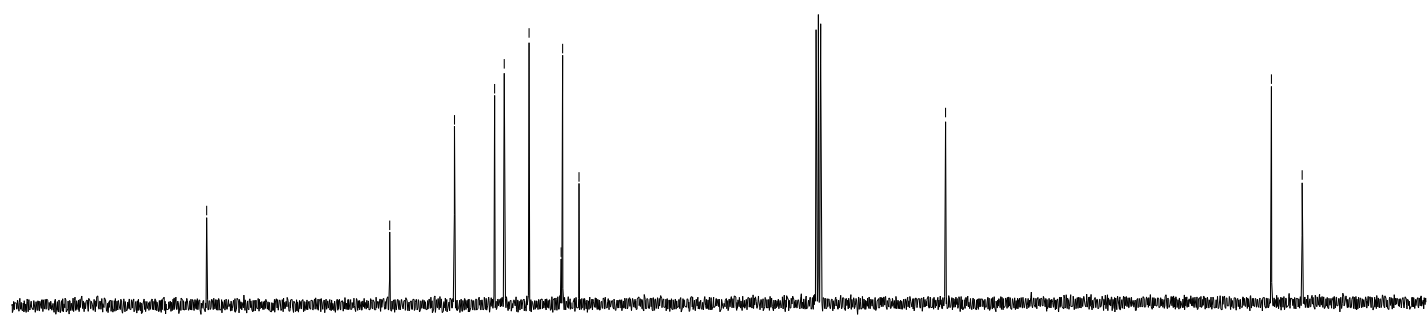

$\begin{array}{lllllllllllllllllll}180 & 170 & 160 & 150 & 140 & 130 & 120 & 110 & 100 \underset{\mathrm{f} 1}{\stackrel{(\mathrm{ppm})}{90}} \mathbf{8 0} & 70 & 60 & 50 & 40 & 30 & 20 & 10 & 0\end{array}$ 
(25) The ${ }^{1} \mathrm{H}$ NMR and ${ }^{13} \mathrm{C}$ NMR spectrum for $\mathbf{3 y}$ (using $\mathrm{CDCl}_{3}$ as solvent)

$$
\text { 守䏍 }
$$

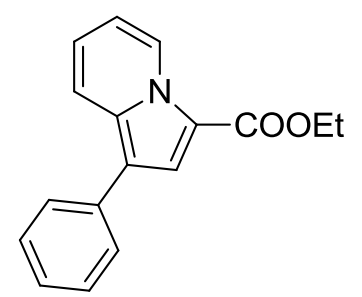

\section{$3 y$}

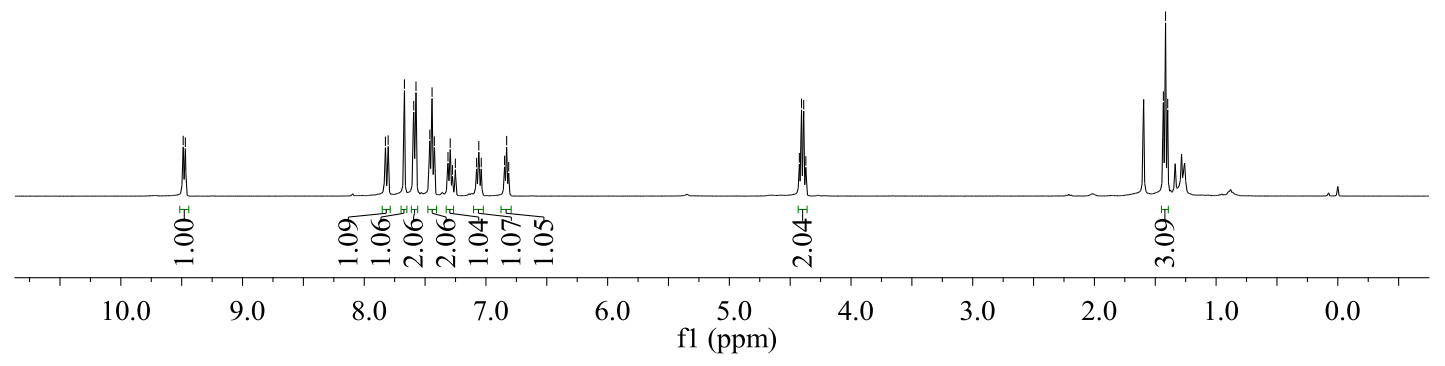

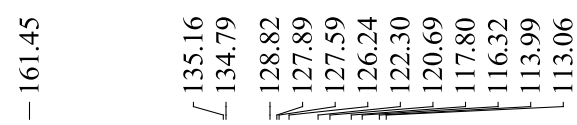
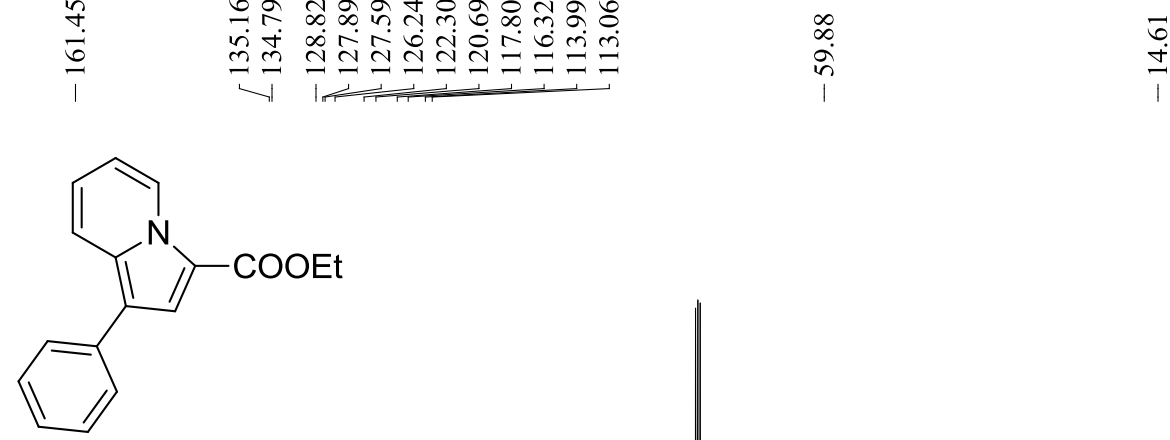

$3 y$

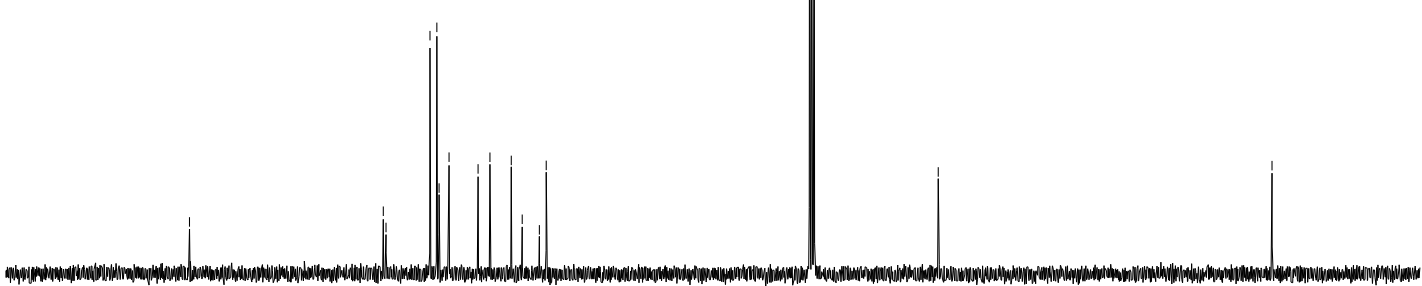

$\begin{array}{llllllllllllllllll}180 & 170 & 160 & 150 & 140 & 130 & 120 & 110 & 100 \underset{\mathrm{f} 1(\mathrm{ppm})}{90} & 80 & 70 & 60 & 50 & 40 & 30 & 20 & 10 & 0\end{array}$ 
(26) The ${ }^{1} \mathrm{H}$ NMR and ${ }^{13} \mathrm{C}$ NMR spectrum for $\mathbf{3 z}$ (using $\mathrm{CDCl}_{3}$ as solvent)

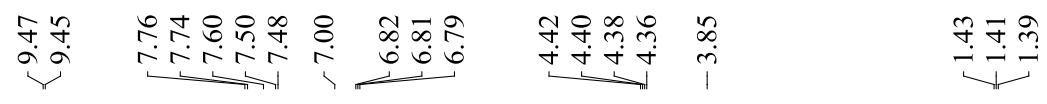
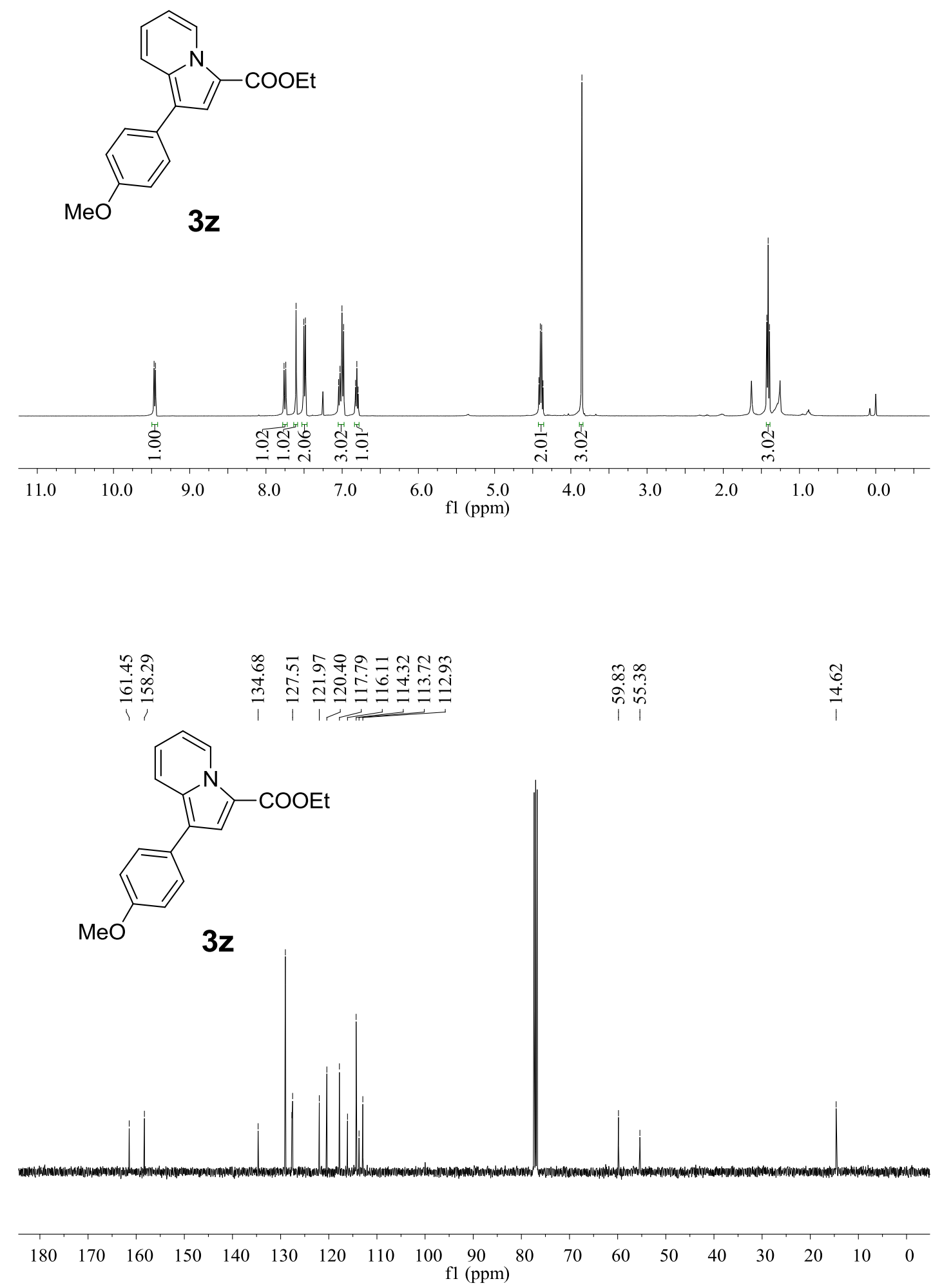
(27) The ${ }^{1} \mathrm{H}$ NMR and ${ }^{13} \mathrm{C}$ NMR spectrum for $\mathbf{3 a z}$ (using $\mathrm{CDCl}_{3}$ as solvent)

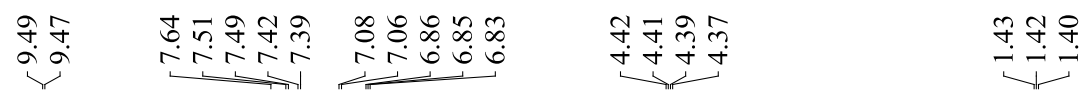

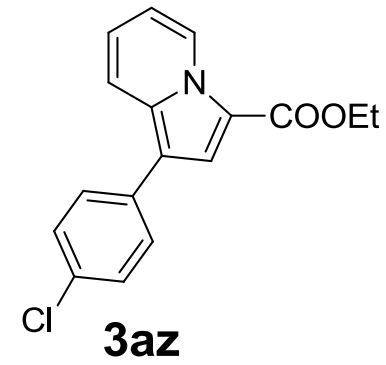

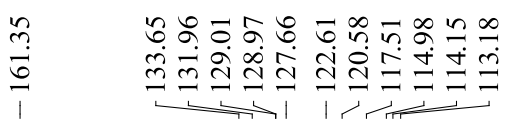
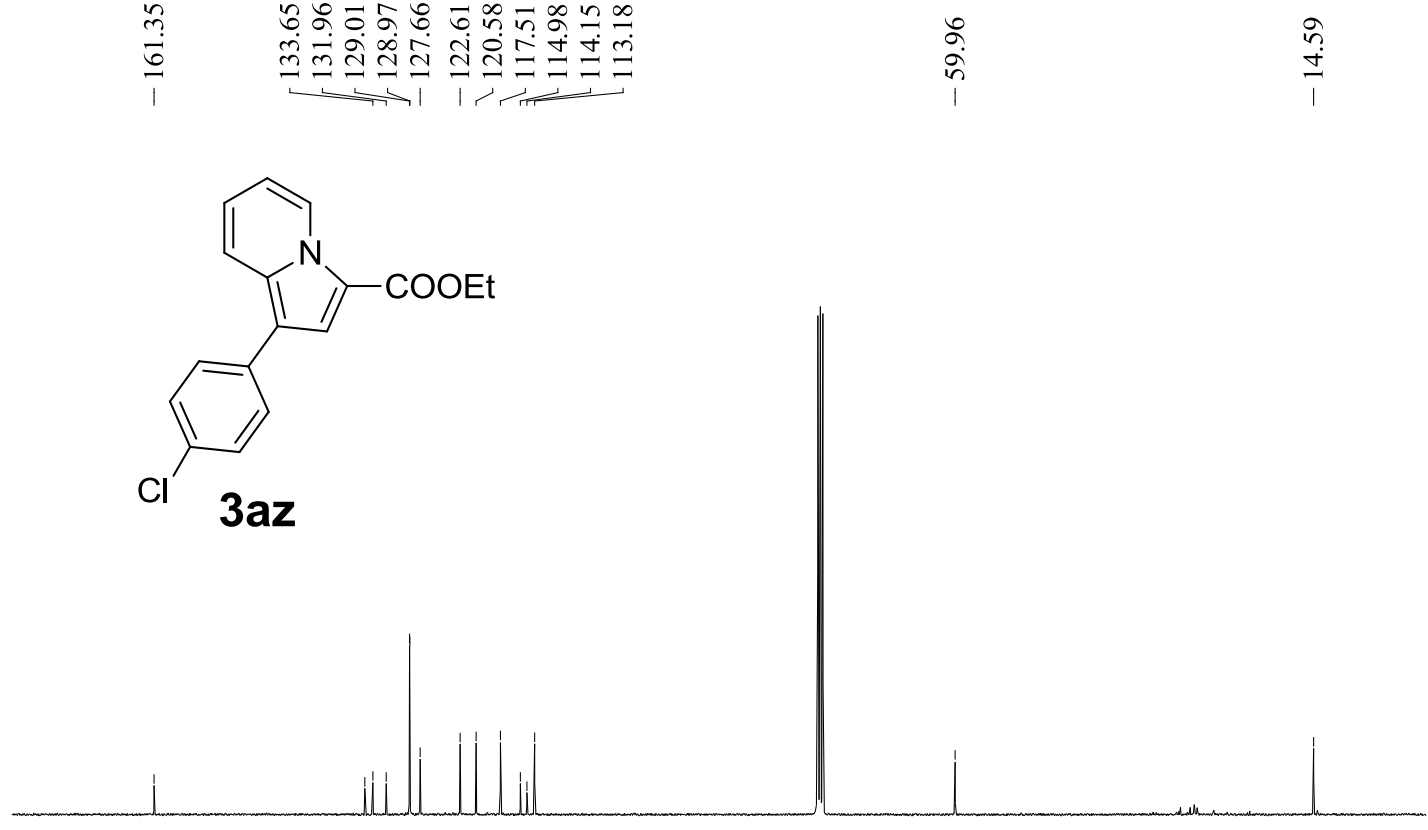

$\begin{array}{lllllllllllllllll}170 & 160 & 150 & 140 & 130 & 120 & 110 & 100 \underset{\mathrm{f} 1(\mathrm{ppm})}{90} & 80 & 70 & 60 & 50 & 40 & 30 & 20 & 10\end{array}$ 
(28) The ${ }^{1} \mathrm{H}$ NMR and ${ }^{13} \mathrm{C}$ NMR spectrum for $\mathbf{3 b z}$ (using $\mathrm{CDCl}_{3}$ as solvent)
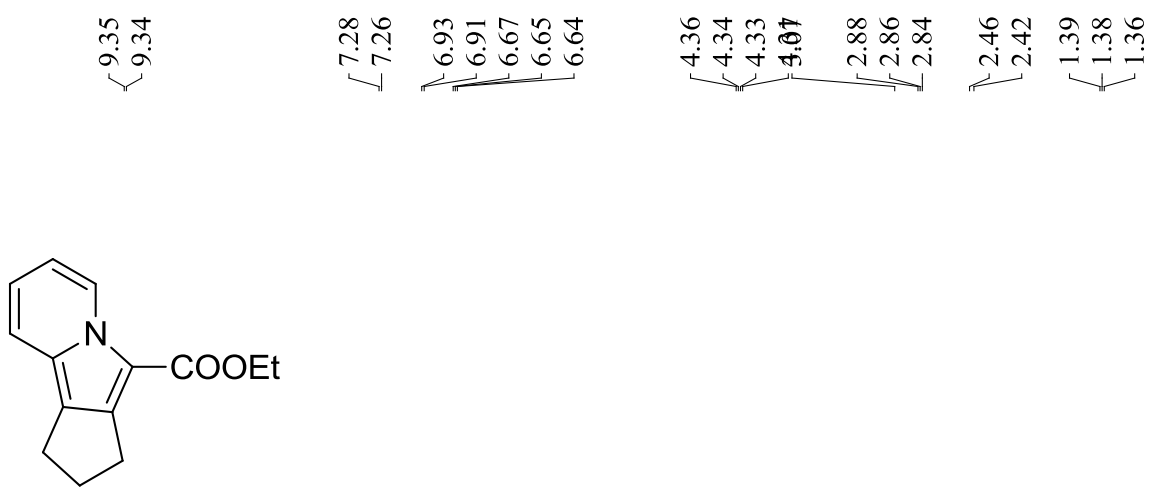

$3 \mathrm{bz}$

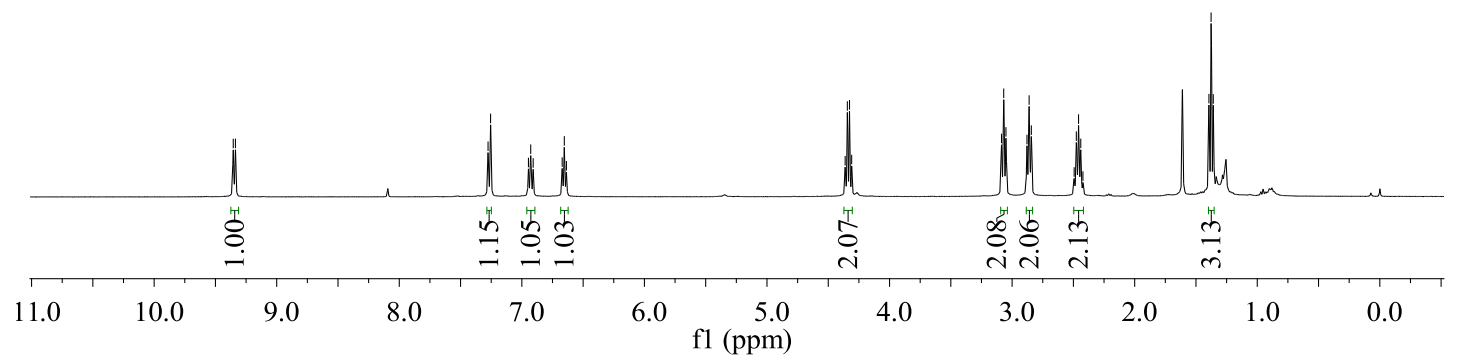

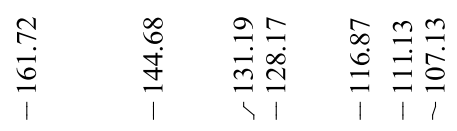

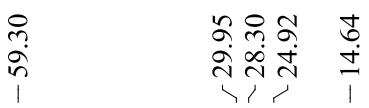

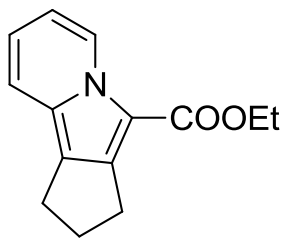

$3 \mathrm{bz}$

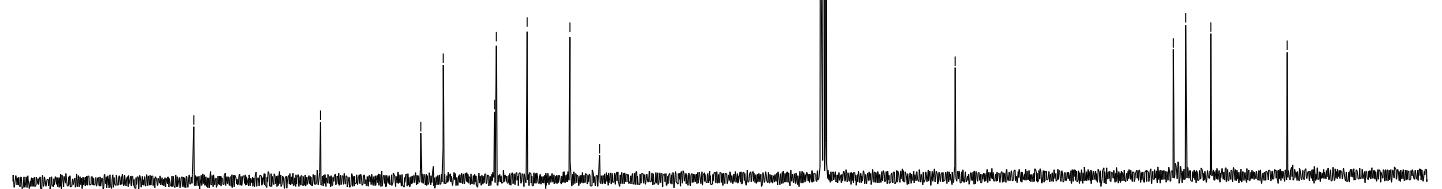

$\begin{array}{lllllllllllllllllll}180 & 170 & 160 & 150 & 140 & 130 & 120 & 110 & 100 & 90 & 80 & 70 & 60 & 50 & 40 & 30 & 20 & 10 & 0\end{array}$ 
(29) The ${ }^{1} \mathrm{H}$ NMR and ${ }^{13} \mathrm{C}$ NMR spectrum for $\mathbf{3 c z}$ (using $\mathrm{CDCl}_{3}$ as solvent)

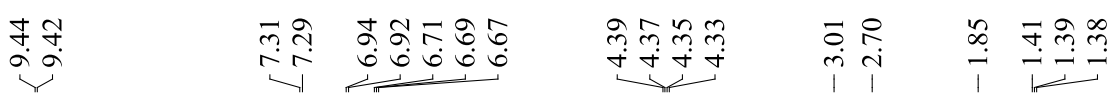

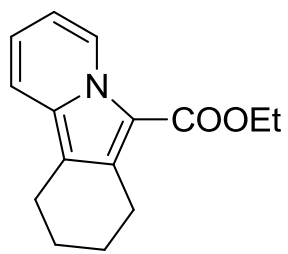

$3 \mathrm{CZ}$

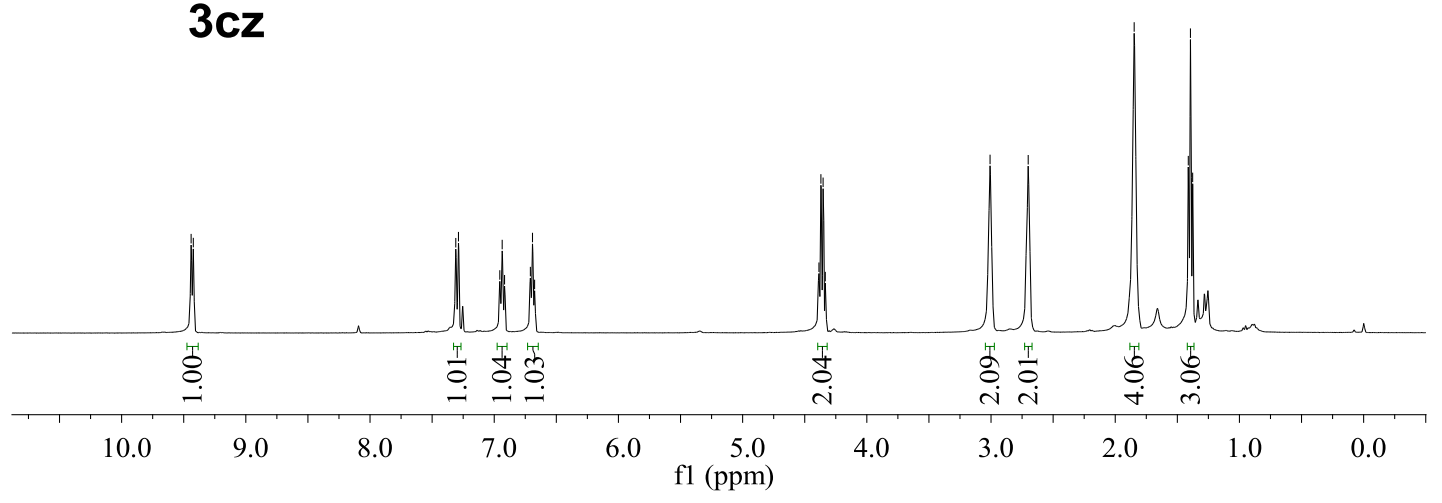

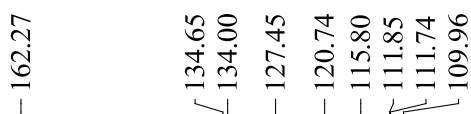

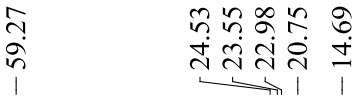

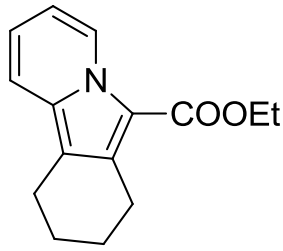

$3 c z$

$\begin{array}{lllllllllllllllllll}180 & 170 & 160 & 150 & 140 & 130 & 120 & 110 & 100 & 90 & 80 & 70 & 60 & 50 & 40 & 30 & 20 & 10 & 0\end{array}$ f1 (ppm) 
(30) The ${ }^{1} \mathrm{H}$ NMR and ${ }^{13} \mathrm{C}$ NMR spectrum for $\mathbf{3 d z}$ (using $\mathrm{CDCl}_{3}$ as solvent)

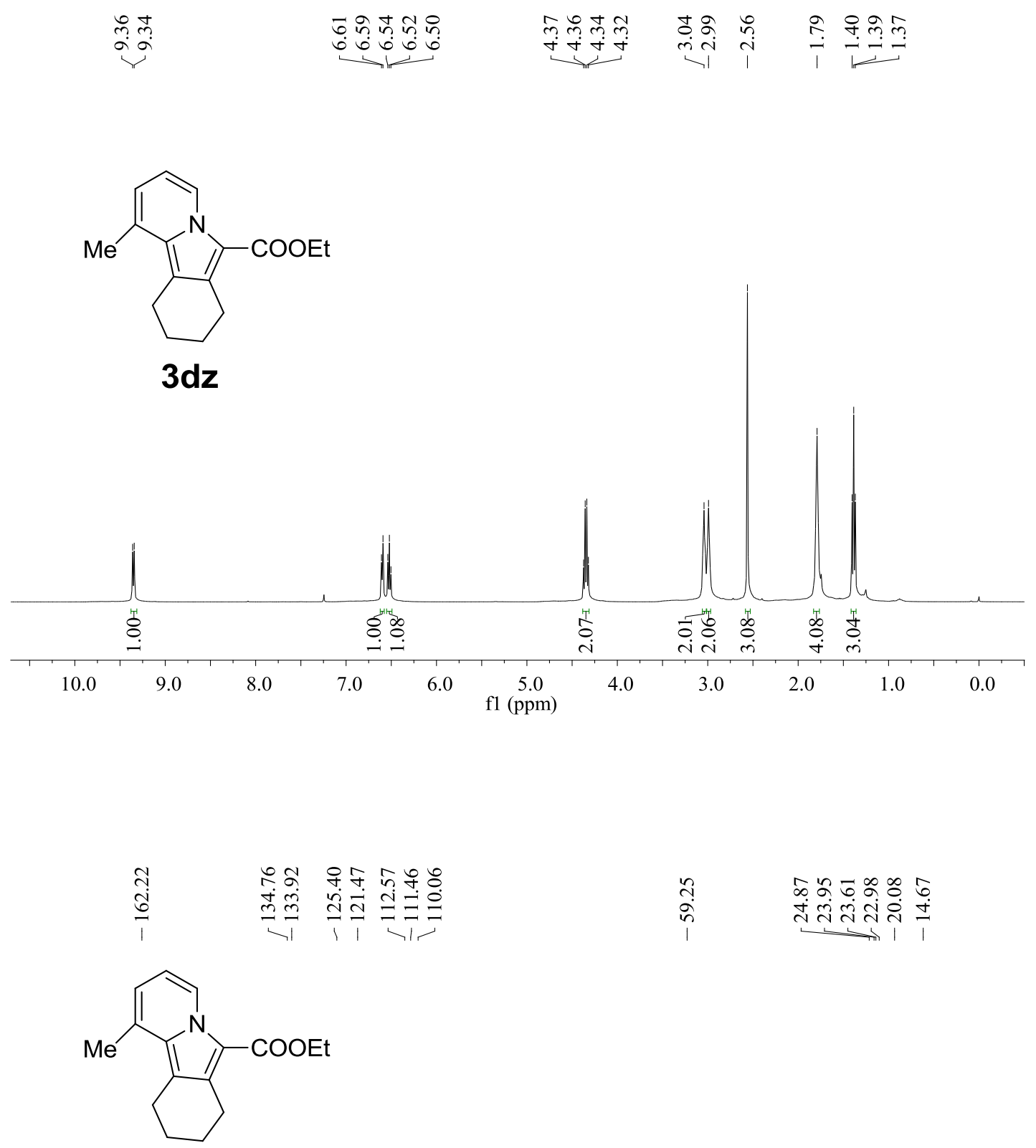

$3 d z$

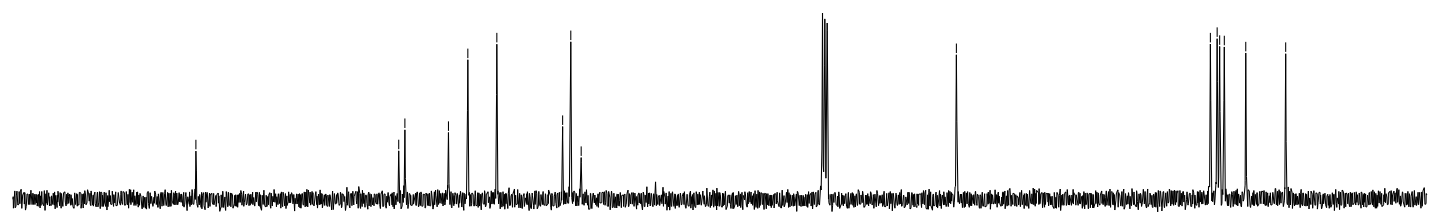

$\begin{array}{llllllllllllllllll}180 & 170 & 160 & 150 & 140 & 130 & 120 & 110 & 100 \underset{\mathrm{f} 1(\mathrm{ppm})}{90} & 80 & 70 & 60 & 50 & 40 & 30 & 20 & 10 & 0\end{array}$ 
(31) The ${ }^{1} \mathrm{H}$ NMR and ${ }^{13} \mathrm{C}$ NMR spectrum for $\mathbf{3 e z}$ (using $\mathrm{CDCl}_{3}$ as solvent)

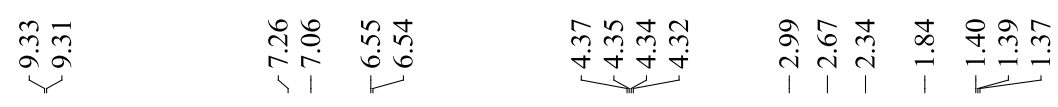

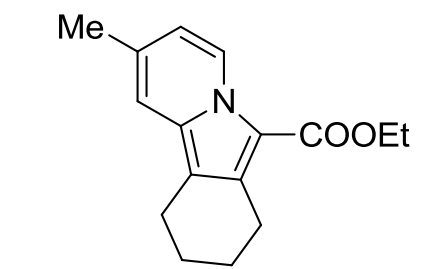

$3 e z$

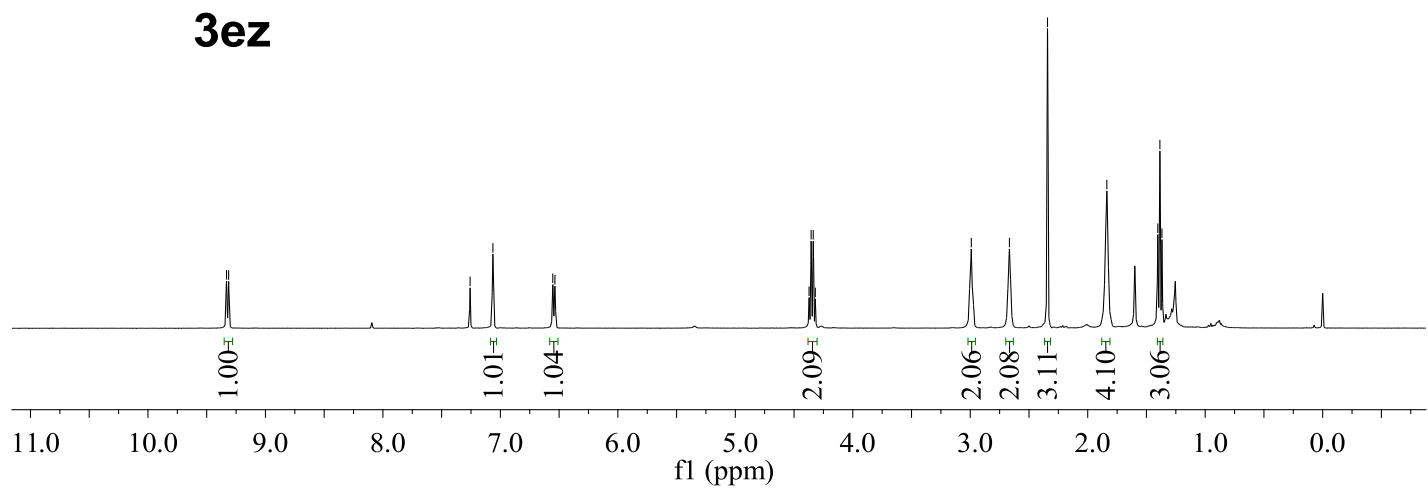

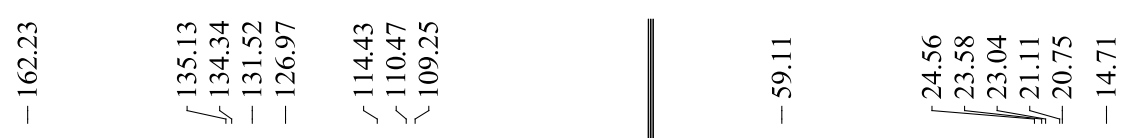<smiles>CCOC(=O)c1c2c(c3cc(C)ccn13)CCCC2</smiles>

$3 e z$

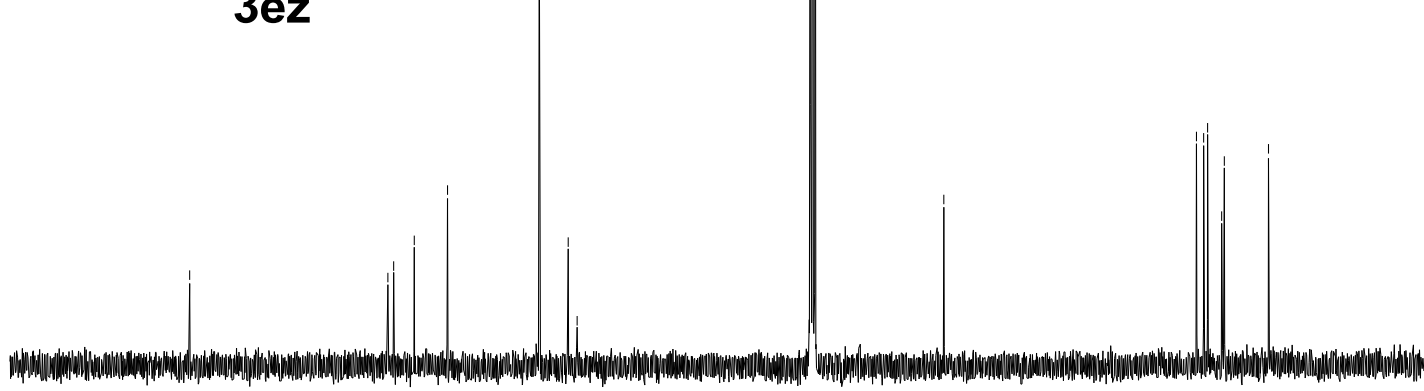

$\begin{array}{llllllllllllllllll}180 & 170 & 160 & 150 & 140 & 130 & 120 & 110 & 100 \underset{\mathrm{f} 1}{(\mathrm{ppm})} & \begin{array}{c}90 \\ (\mathrm{ppm})\end{array} & 70 & 60 & 50 & 40 & 30 & 20 & 10 & 0\end{array}$ 
(32) The ${ }^{1} \mathrm{H}$ NMR and ${ }^{13} \mathrm{C}$ NMR spectrum for $\mathbf{3 f z}$ (using $\mathrm{CDCl}_{3}$ as solvent)
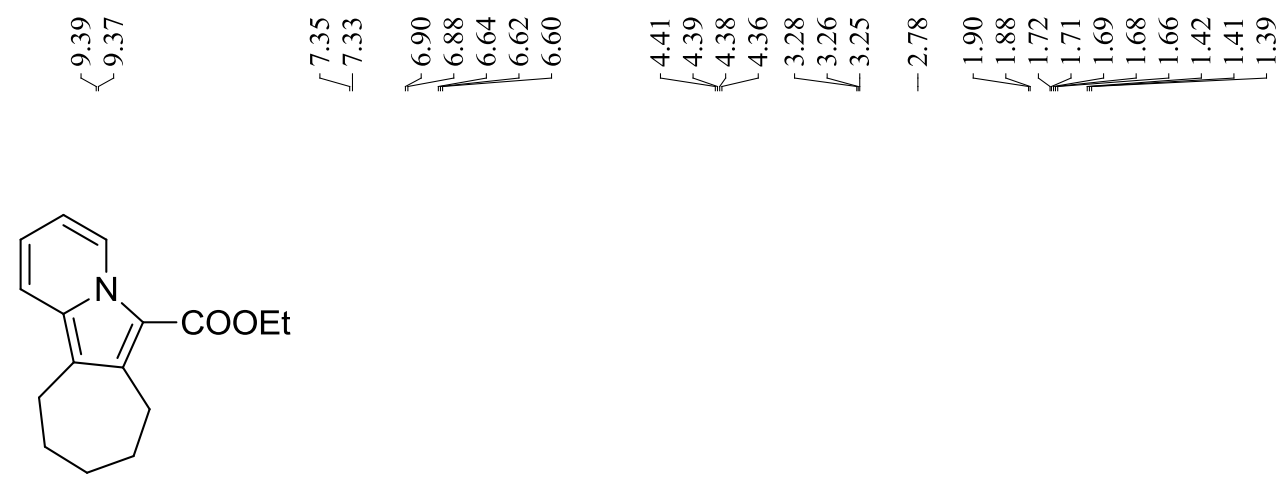

3fz

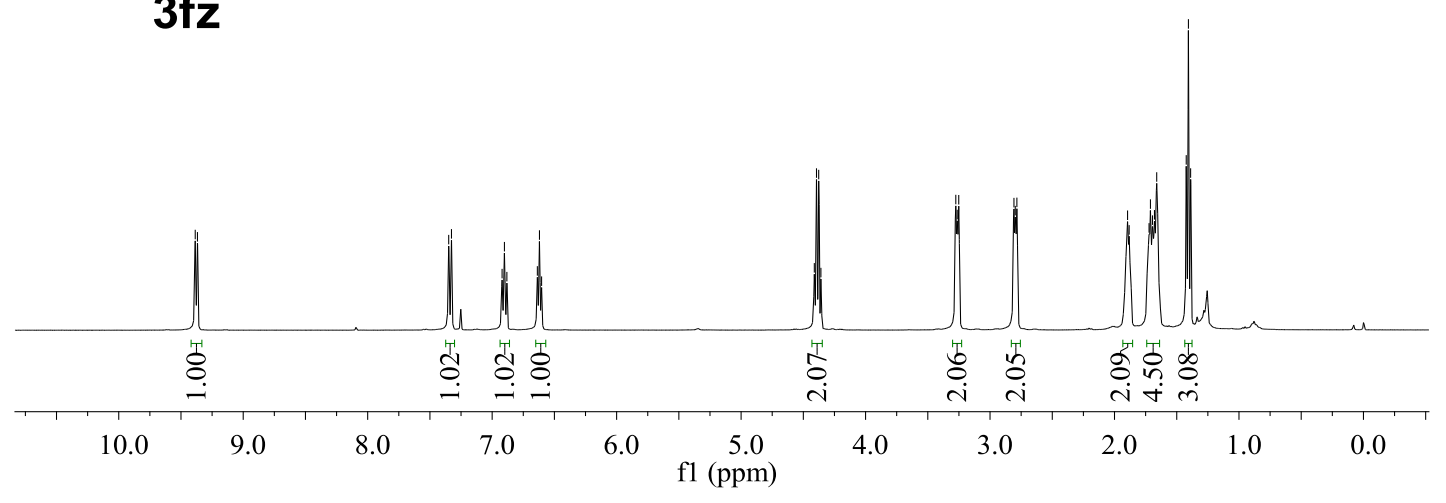

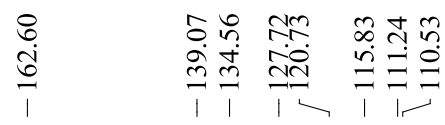

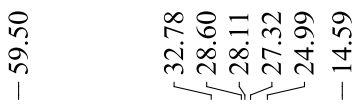

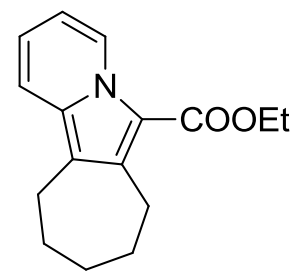

3fz

$\begin{array}{lllllllllllllllllll}190 & 180 & 170 & 160 & 150 & 140 & 130 & 120 & 110 & \begin{array}{c}100 \\ \mathrm{f} 1(\mathrm{ppm})\end{array} & \begin{array}{c}90 \\ (\mathrm{ppm})\end{array} & 70 & 60 & 50 & 40 & 30 & 20 & 10 & 0\end{array}$ 
(33) The ${ }^{1} \mathrm{H}$ NMR and ${ }^{13} \mathrm{C}$ NMR spectrum for $\mathbf{3 g z}$ (using $\mathrm{CDCl}_{3}$ as solvent)

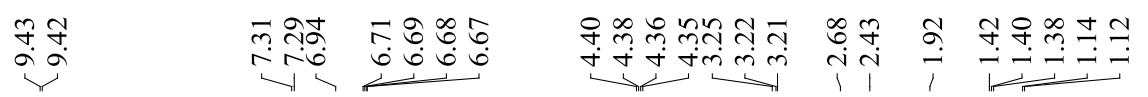<smiles>CCOC(=O)c1c2c(c3ccccn13)CCC(C)C2</smiles>

Me

$3 \mathrm{gz}$

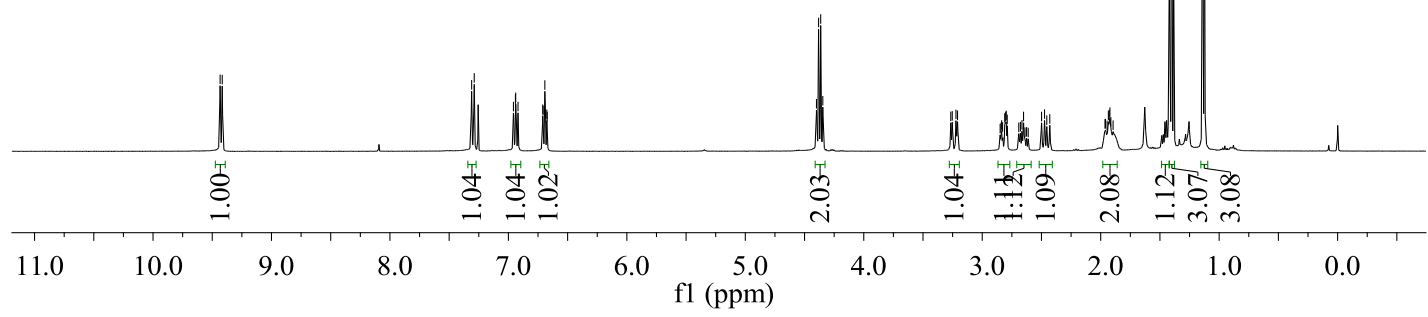

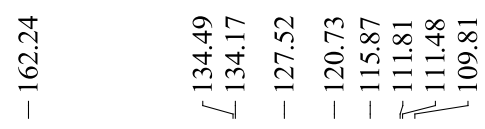

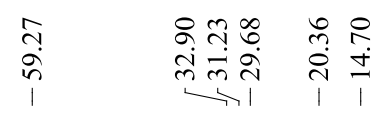

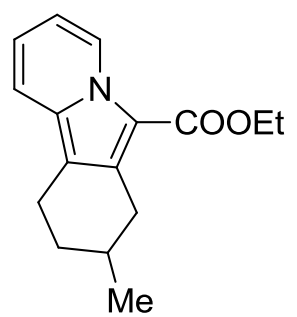

$3 g z$

$\begin{array}{lllllllllllllllllll}180 & 170 & 160 & 150 & 140 & 130 & 120 & 110 & 100 \underset{\mathrm{f} 1(\mathrm{ppm})}{90} & 80 & 70 & 60 & 50 & 40 & 30 & 20 & 10 & 0\end{array}$ 
(34) The ${ }^{1} \mathrm{H}$ NMR and ${ }^{13} \mathrm{C}$ NMR spectrum for $\mathbf{3 h z}$ (using $\mathrm{CDCl}_{3}$ as solvent)
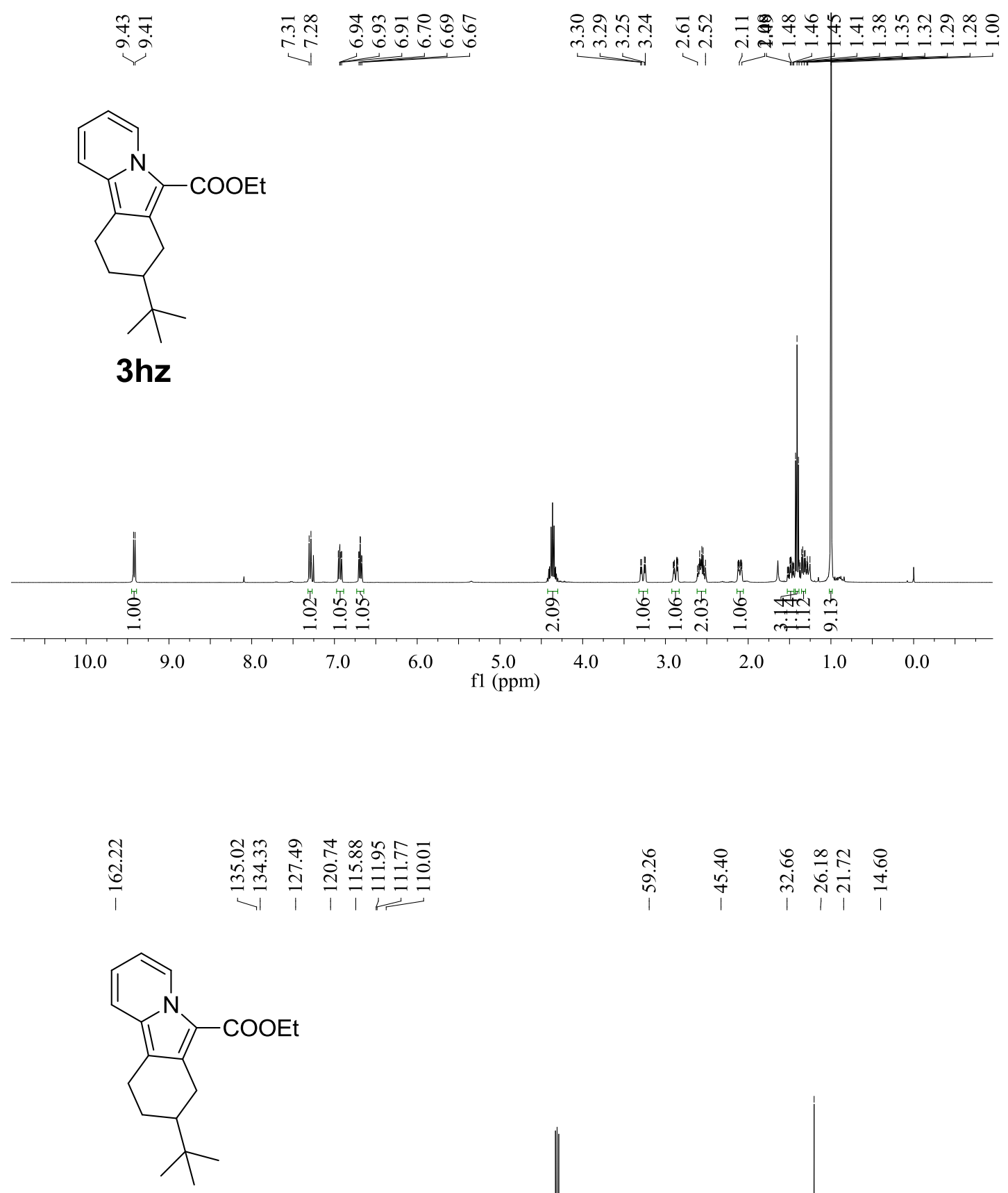

3hz

$\begin{array}{lllllllllllllllllll}180 & 170 & 160 & 150 & 140 & 130 & 120 & 110 & 100 & 90 & 80 & 70 & 60 & 50 & 40 & 30 & 20 & 10 & 0\end{array}$ 
(35) The ${ }^{1} \mathrm{H}$ NMR and ${ }^{13} \mathrm{C}$ NMR spectrum for $3 i z$ (using $\mathrm{CDCl}_{3}$ as solvent)

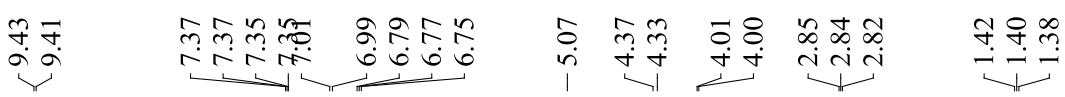<smiles>CCOC(=O)c1c2c(c3ccccn13)CCOC2</smiles>

$3 i z$

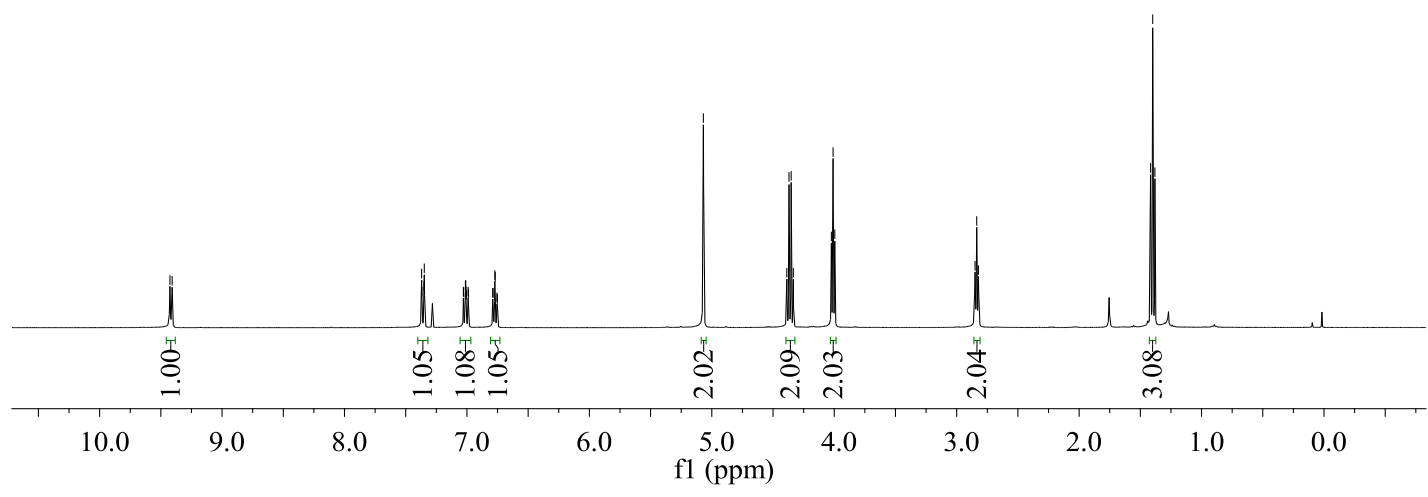

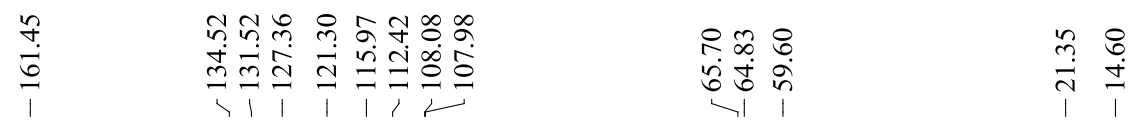<smiles>CCOC(=O)c1c2c(c3ccccn13)CCOC2</smiles>

3iz

$\begin{array}{lllllllllllllllllll}180 & 170 & 160 & 150 & 140 & 130 & 120 & 110 & 100 & 90 & 80 & 70 & 60 & 50 & 40 & 30 & 20 & 10 & 0\end{array}$ 
(36) The ${ }^{1} \mathrm{H}$ NMR and ${ }^{13} \mathrm{C}$ NMR spectrum for $\mathbf{3 j z}$ (using $\mathrm{CDCl}_{3}$ as solvent)

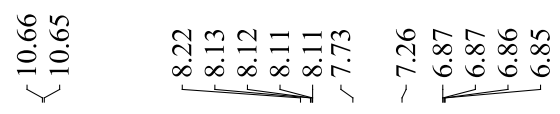

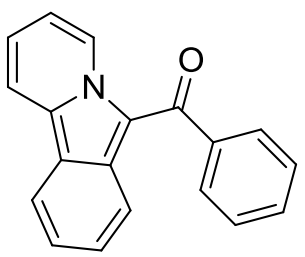

\section{3jz}
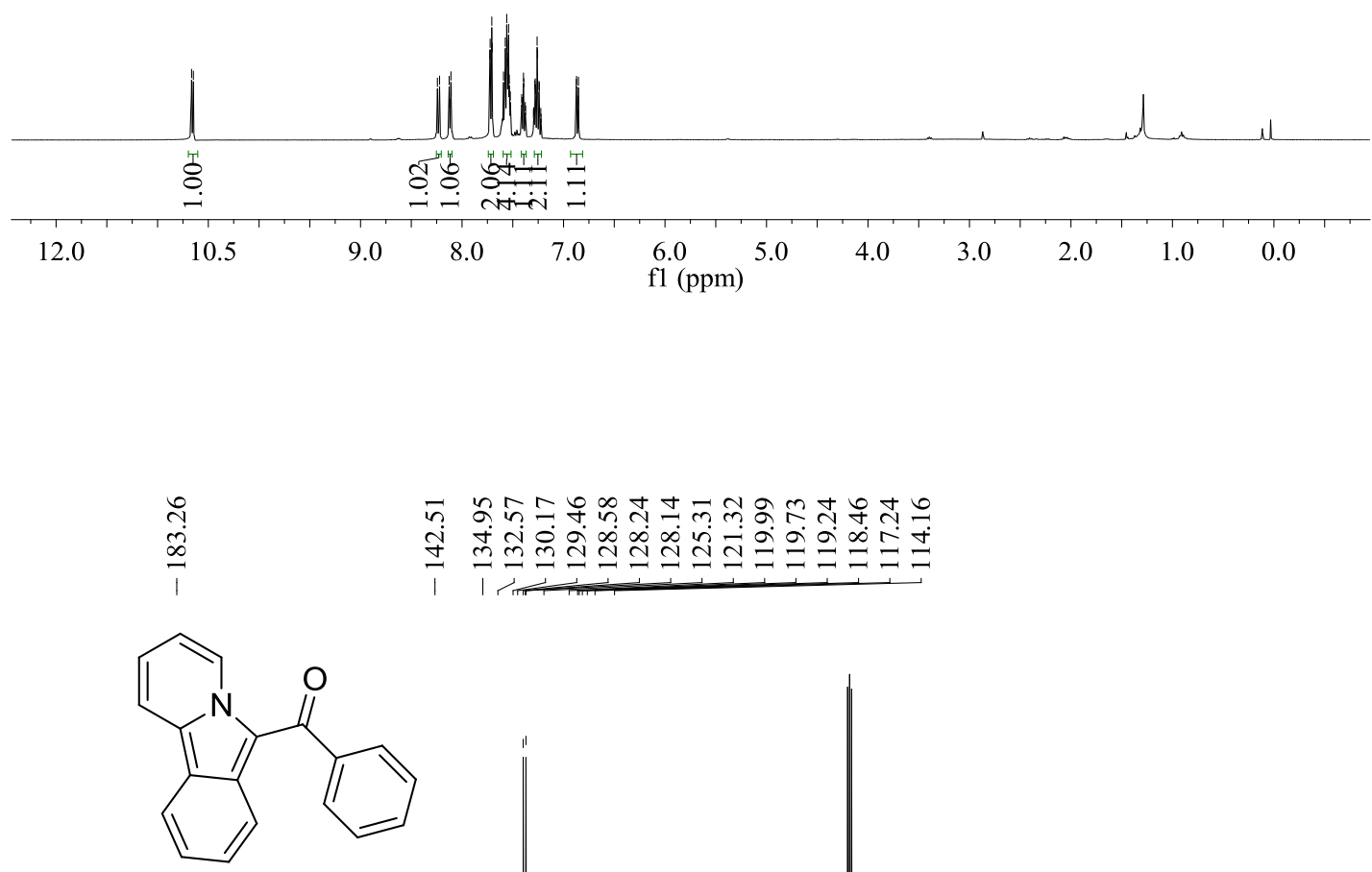

$3 j z$

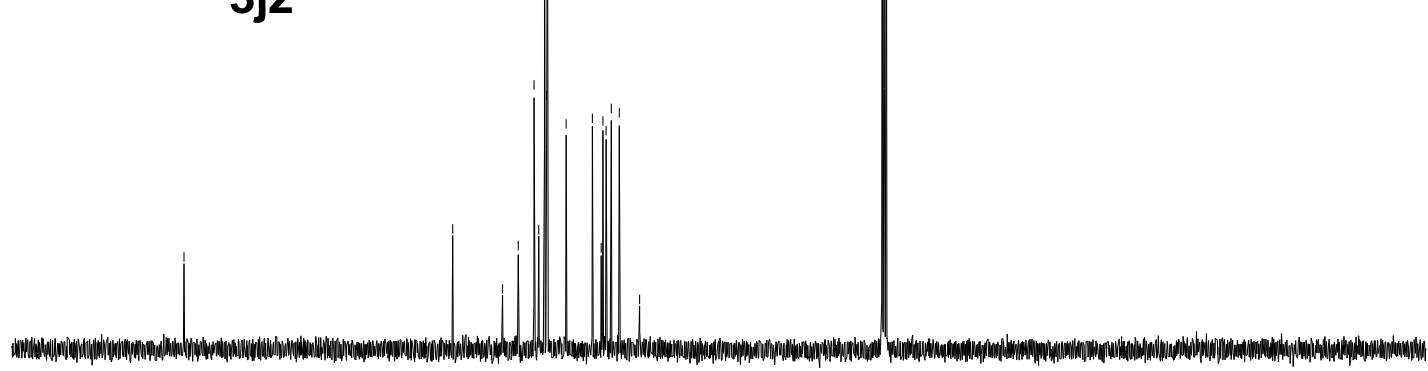

190

170

150

130

110
$\mathrm{f} 1$

$\begin{array}{llllllllll}90 & 80 & 70 & 60 & 50 & 40 & 30 & 20 & 10 & 0\end{array}$ 
(37) The ${ }^{1} \mathrm{H}$ NMR and ${ }^{13} \mathrm{C}$ NMR spectrum for $\mathbf{3 k z}$ (using $\mathrm{CDCl}_{3}$ as solvent)

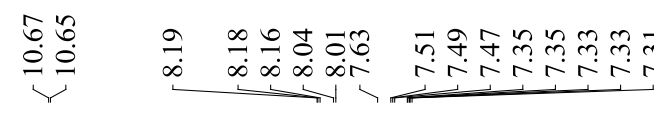<smiles>CC(=O)c1c2ccccc2c2ccccn12</smiles>

$3 k z$
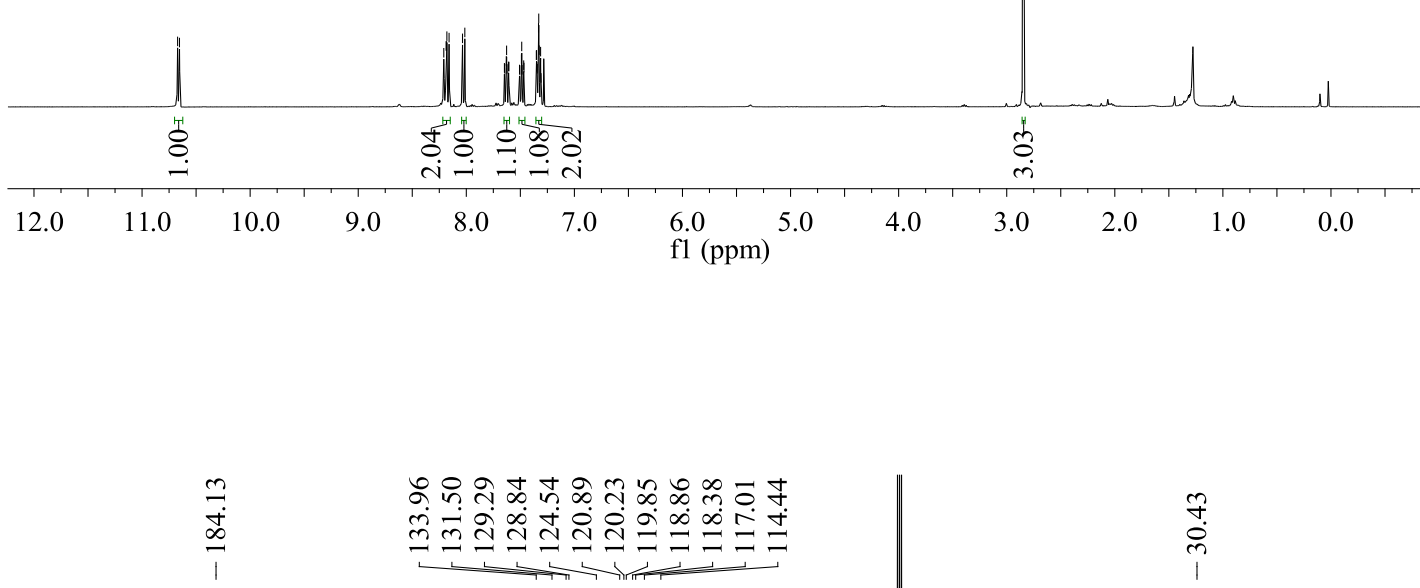<smiles>CC(=O)c1c2ccccc2c2ccccn12</smiles>

$3 \mathrm{kz}$
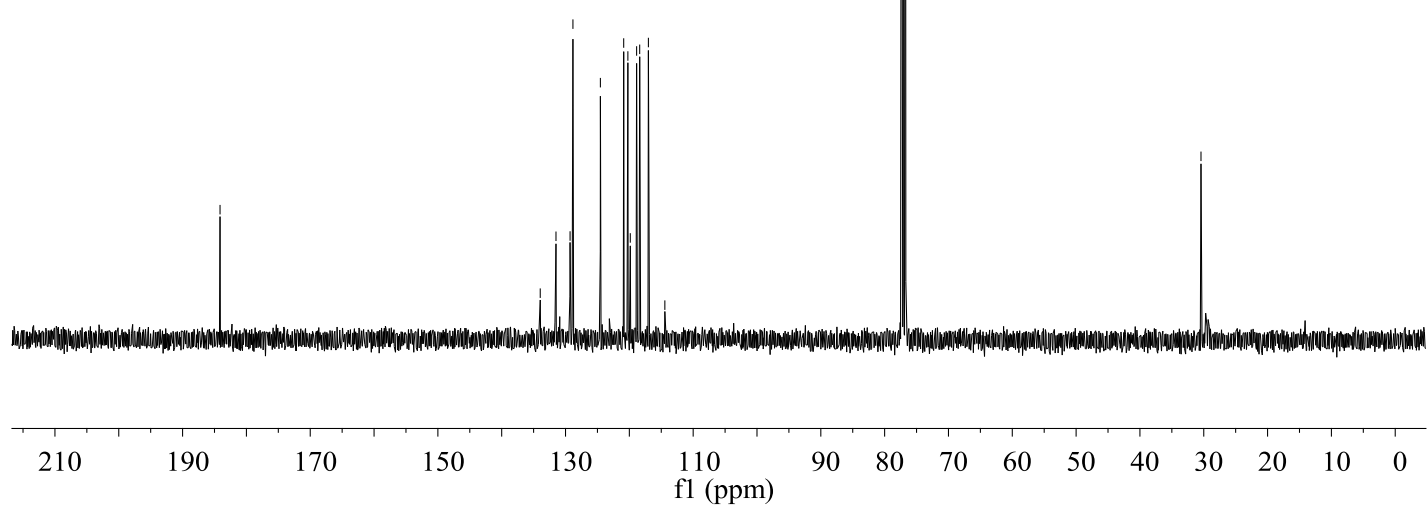
(38) The ${ }^{1} \mathrm{H}$ NMR and ${ }^{13} \mathrm{C}$ NMR spectrum for $4 \mathbf{a}$ (using $\mathrm{CDCl}_{3}$ as solvent)

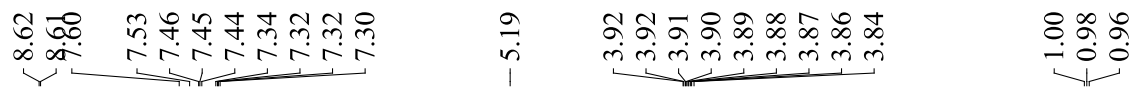<smiles>CCOC(=O)C(O)c1ccccc1-c1ccccn1</smiles>

$4 a$

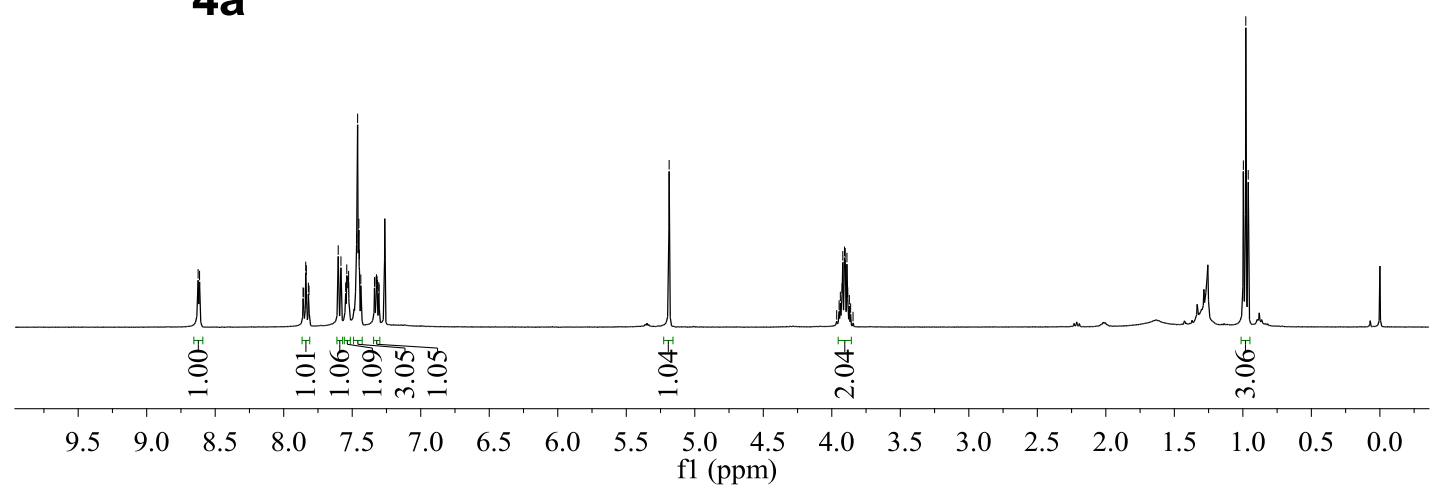

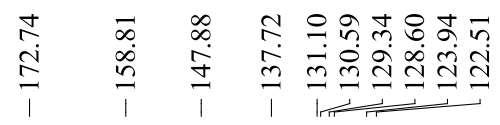

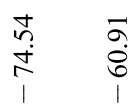

$\underset{\vec{a}}{\vec{n}}$<smiles>CCOC(=O)C(O)c1ccccc1-c1ccccn1</smiles>

$4 a$

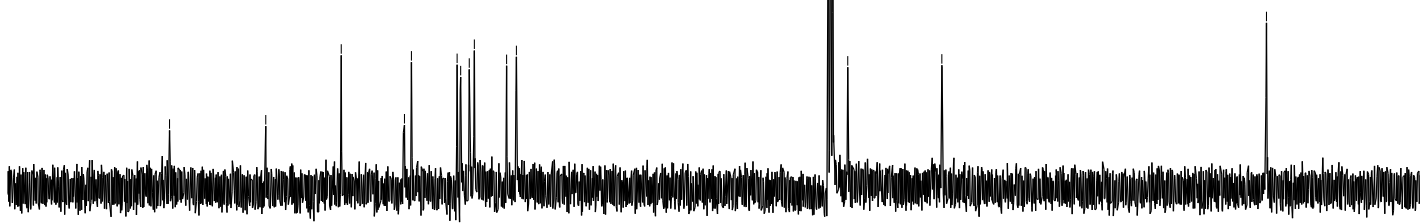

190

170

150

130

110

f1 (ppm)

$\begin{array}{lllllllll}80 & 70 & 60 & 50 & 40 & 30 & 20 & 10 & 0\end{array}$ 
(39) The ${ }^{1} \mathrm{H}$ NMR and ${ }^{13} \mathrm{C}$ NMR spectrum for $\boldsymbol{d}$-1a (using $\mathrm{CDCl}_{3}$ as solvent)

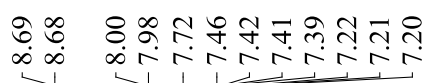
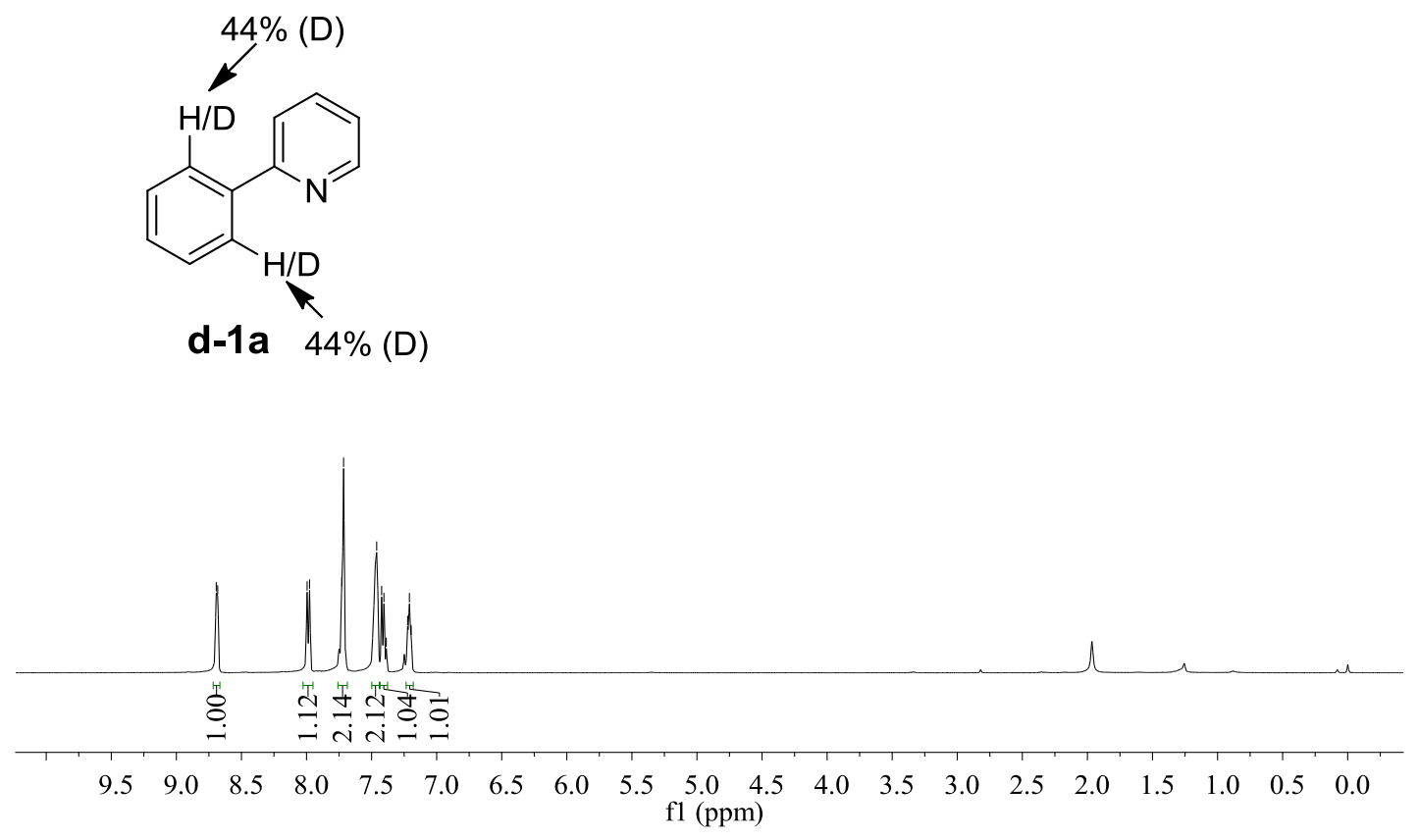

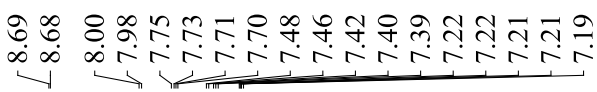

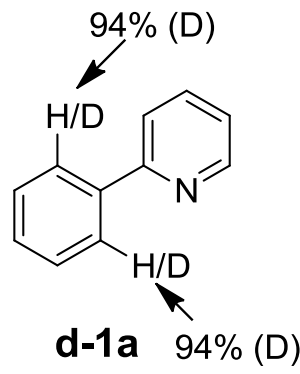

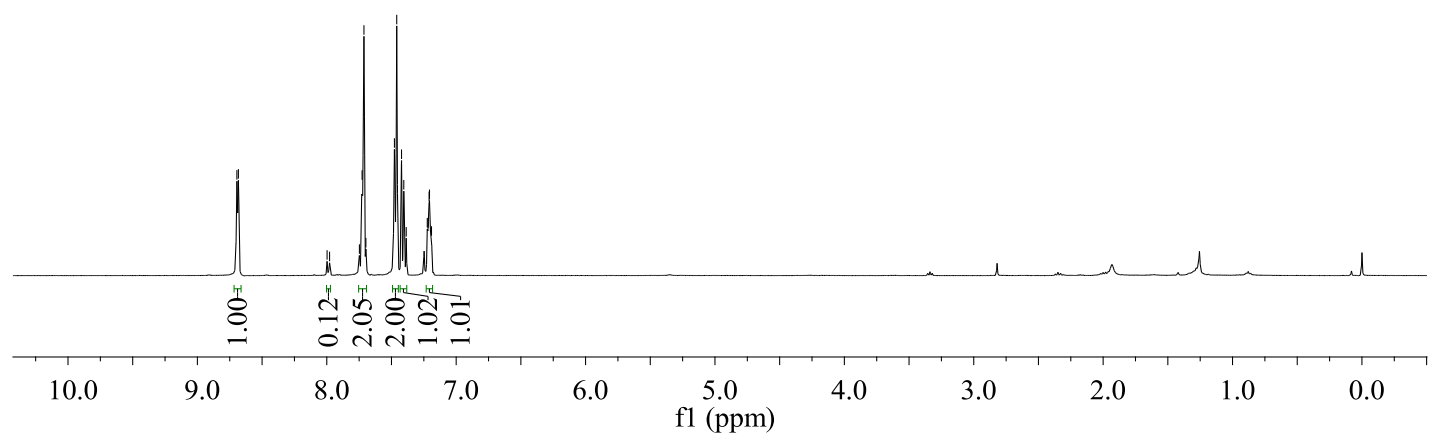


(40) The ${ }^{1} \mathrm{H}$ NMR and ${ }^{13} \mathrm{C}$ NMR spectrum for $\mathbf{d - 3 a}$ (using $\mathrm{CDCl}_{3}$ as solvent)

$$
\text { 苛 }
$$

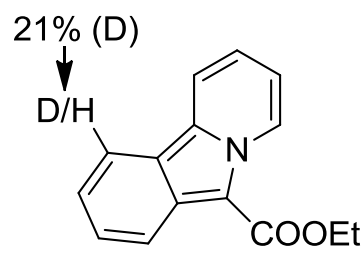

\section{d-3a}
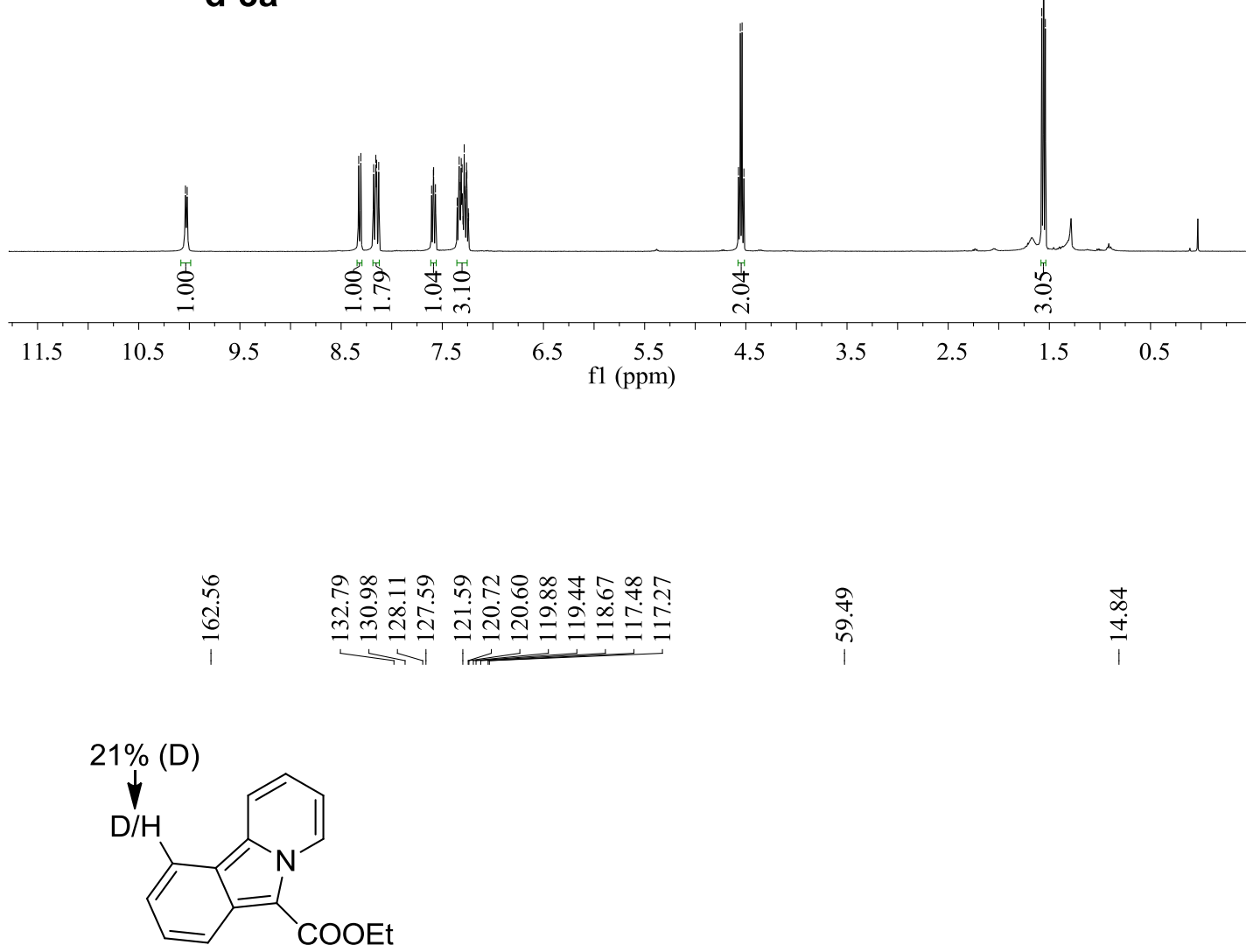

d-3a

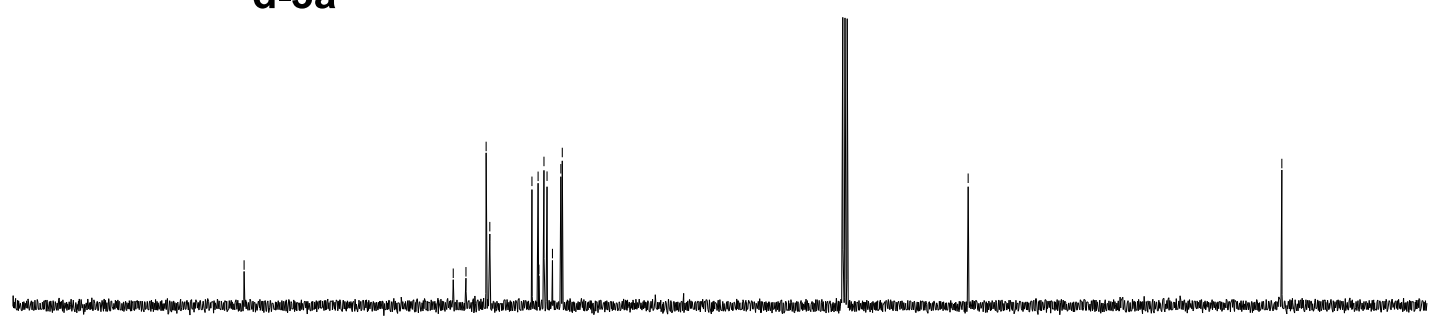

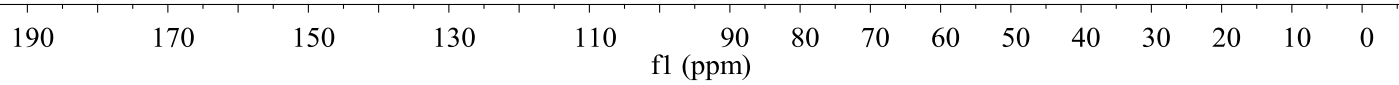

